\title{
Equilibrium Fluctuations for a Model of Coagulating-Fragmenting Planar Brownian Particles
}

\author{
Mojtaba Ranjbar ${ }^{1}$, Fraydoun Rezakhanlou ${ }^{2, \star}$ \\ 1 Mathematics and Computer Science Faculty, Amirkabir University, Tehran, Iran \\ 2 Department of Mathematics, University of California, Berkeley, \\ California 94720-3830, USA. E-mail: rezakhan@math.berkeley.edu
}

Received: 29 May 2009 / Accepted: 24 November 2009

Published online: 18 February 2010 - (C) The Author(s) 2010. This article is published with open access at Springerlink.com

\begin{abstract}
We study a model of mass-bearing coagulating-fragmenting planar Brownian particles. Coagulation occurs when two particles are within a distance of order $\varepsilon$. Our model is macroscopically described by an inhomogeneous Smoluchowski's equation in the low $\varepsilon$ limit provided that the initial number of particles $N$ is of order $|\log \varepsilon|$. When a detailed balance condition is satisfied, we establish a central limit theorem by showing that in the low $\varepsilon$ limit, the particle density fluctuation fields obey an Ornstein-Uhlenbeck stochastic differential equation.
\end{abstract}

\section{Introduction}

One of the main purposes of statistical mechanics is to explain the macroscopic behavior of various phenomena in terms of the statistics of their microscopic structures. Macroscopically we often have a PDE involving a small number of parameters. The microscopic description however involves a large number of components that are evolving by either deterministic or stochastic rules. Let us name three reasons to justify our interest in understanding the connection between the microscopic and macroscopic descriptions.

As our first reason, we remark that historically the macroscopic equation is formally derived from the microscopic description of the phenomenon under study. It is an important task of statistical mechanics to justify such a derivation rigorously and verify the validity of the macroscopic PDE.

For our second reason, we note that we often have simple dynamical rules for the microscopic model and the main challenging feature of the model has to do with its large size. On the other hand, the macroscopic evolution involves only a few variables but is dictated by a rather sophisticated nonlinear rules. It is the case for many examples that the macroscopic equation is not fully understood. Hopefully by exploring its relation with its microscopic counterpart we may discover new tools and techniques for the macroscopic equation.

\footnotetext{
* This work is supported in part by NSF grant DMS-0707890.
} 
As our third reason, we should mention that even though the macroscopic equation is preferred because of its dependence on a small number of variables, it is only a reduced description of the microscopic phenomenon at hand and we would like to find practical ways of recovering some of the lost information as we switch to the macroscopic world. Since the passage from the microscopic details to macroscopic parameters can be recast as a law of large numbers for some conserved quantities in many scenarios, probability theory suggests some standard routes for going beyond the law of large numbers and gain new information. The celebrated central limit theorem and large deviations for classical examples are guidelines for producing some vital information for the microscopic model under study.

It is the latter reason which is the chief motivation for the present article. Our microscopic model is a system of coagulating-fragmenting Brownain particles which is macroscopically described by an inhomogeneous Smoluchowski equation. This equation is derived as a law of large numbers. The main contribution of this article is a central limit theorem for the aforementioned law of large numbers when the system is in equilibrium.

In our model, we start with $N$ particles with each particle traveling in $\mathbb{R}^{d}$ as a Brownain motion. Each particle has a size $m \in \mathbb{Z}$ and a radius $r \in(0, \infty)$. In fact our interpretation of the location $x$ of a particle is that $x$ is the center of a ball of radius $r$ and in some sense only a small fraction of the ball is occupied by the true particle. It turns out that in reality each particle is a cluster of smaller objects and the cluster is a complex fractal like entity that is too complicated to be treated with the existing techniques. That is why we simplify the model by replacing the cluster with a ball of radius $r(m)=m^{\chi}$ so that when $\chi>\frac{1}{d}$, we are taking into account the fact that the mass of the particle comes from a small portion of the ball which is occupied by the cluster. This may appear somewhat native and not too realistic from a physical point of view. Nevertheless, as was explained in [HR1-3] and [R2], the model does exhibit some expected features of the underlying physics. For example, the condition $\chi<\frac{1}{d-2}$ guarantees that no gelation occurs in finite time. That is, no particle of size infinity is formed in finite time at the macroscopic level. We also conjecture that an instantaneous gelation would occur if $\chi>\frac{1}{d-2}$.

In fact the true radius of a particle is $\varepsilon r$ with $\varepsilon$ very small. A calculation involving Wiener sausages reveals that if $N=\varepsilon^{2-d}$ when $d \geq 3$ and $N=|\log \varepsilon|$ when $d=2$, then the expected value of the number of times a particle coagulates with other particles in one unit of time stays positive and finite as $\epsilon \rightarrow 0$. This property allows us to obtain the Smoluchowski's equation for the evolution of cluster densities in the low $\varepsilon$ limit. To further simplify the involved mathematical technicalities, we forget about balls presenting each particle and regard each particle as a point. Now the coagulation occurs stochastically only when particles of positions $x$ and $y$ and masses $m$ and $n$, satisfy

$$
|x-y| \leq c_{0} \varepsilon\left(m^{\chi}+n^{\chi}\right),
$$

for a constant $c_{0}$. In the preceding works [HR1-2], [R2 and HRY], we were able to derive the macroscopic equation as a law of large numbers; if we label the locations and masses of particles as $\left(x_{i}, m_{i}\right), i \in I$, then our law of large numbers asserts

$$
\frac{1}{K_{\varepsilon}} \sum_{i \in I} \delta_{x_{i}(t)}(d x) \mathbb{1}\left(m_{i}(t)=n\right) \rightarrow f_{n}(x, t) d x
$$

with $f_{n}$ solving Smoluchowski equation (2.6) of Sect. 2. Here

$$
K_{\varepsilon}= \begin{cases}\varepsilon^{2-d} & \text { if } d \geq 3 \\ |\log \varepsilon| & \text { if } d=2\end{cases}
$$


As a central limit theorem, we are interested in the limit of the fluctuation fields

$$
\xi_{n}^{\varepsilon}(d x, t)=\sqrt{K_{\varepsilon}}\left(K_{\varepsilon}^{-1} \sum_{i} \delta_{x_{i}(t)}(d x) \mathbb{1}\left(m_{i}(t)=n\right)-f_{n}(x, t) d x\right)
$$

as $\varepsilon \rightarrow 0$. In Sect. 2, we state a conjecture regarding the evolution of $\xi_{n}^{\varepsilon}$ in low the $\varepsilon$ limit. According to this conjecture, the limit $\xi$ solves an Ornstein-Uhlenbeck stochastic differential equation in an infinite dimensional setting with $\xi$ living in a negative Sobolev space. The conjecture is formulated using the so-called fluctuation-dissipation principle of non-equilibrium statistical mechanics. In this article, we establish the conjecture only when the dimension is 2 and the model satisfies a detailed-balance condition. Some steps of our proof do not apply to higher dimensions. The case $d \geq 3$ is more challenging and is left for a future investigation.

We continue this Introduction by mentioning some previous work related to our model. Smoluchowski's equation was introduced by Smoluchowski in the seminal work [Sm]. The first mathematically rigorous derivation of Smoluchowski's equation from a model of coagulating Brownian particles was carried out by Lang-Nyugen [LN] when $d=3$ and all particles have the same diffusion coefficient. A related problem has been studied by Sznitman [Sz] when $d=2$. A completely different approach has been employed in [HR1-2 and YRH] to treat the model in general. A thorough survey on related models and their applications can be found in Aldous [A]. In fact Open Problem 16 in $[\mathrm{A}]$ is exactly our central limit theorem when there is no spacial dependence. We refer to the monograph [Sp] for an introduction to related questions in statistical mechanics and a discussion of the fluctuation-dissipation principle. An equilibrium fluctuation result has been studied in [R1] for a model of the colliding particles associated with the discrete Boltzmann equation.

We end this section with an outline of the paper. In Sect.2, we state a conjecture for the macroscopic evolution of the fluctuation fields. In Sect. 3, a family of reversible invariant measures for the microscopic model is constructed. In this section, the conjecture is restated as the main result of this paper under the assumption that the model starts from one of the reversible measures and that the dimension is 2. In Sect. 4, the strategy of the proof is described. The first step of the proof is a regularity of the coagulation term and is carried out in Sects.5-7. The proof of the main result is completed in Sects. 8 and 9 .

\section{A Conjecture}

We start with the description of our model. The configuration space $\Omega$ consists of pairs $\omega=(\mathbf{x}, \mathbf{m})$ with $\mathbf{x}$ a subset of $\mathbb{R}^{d}$ with no accumulation point and $\mathbf{m}: \mathbf{x} \rightarrow \mathbb{N}=$ $\{1,2,3, \ldots\}$ is a map that assigns a positive integer to each element of $\mathbf{x}$. Throughout this section we assume that $d \geq 2$. It is often convenient to write $\omega=\left(x_{i}, m_{i}\right)_{i \in I}$ with $x_{i} \in \mathbb{R}^{d}$ and $m_{i} \in \mathbb{N}$, where $I=I(\omega)$ is a countable index set. We regard $\mathbf{x}$ as a collection of cluster positions in $\mathbb{R}^{d}$ with no accumulation point and $\mathbf{m}$ assigns a size to each such position. We may also identify $\omega=(\mathbf{x}, \mathbf{m})$ as a discrete measure $\hat{\omega}=\sum_{i \in I} \delta_{\left(x_{i}, m_{i}\right)}$ on $\mathbb{R}^{d} \times \mathbb{N}$. Using this identification we equip the space $\Omega$ with the topology of vague convergence.

We now describe the evolution of coagulating and fragmenting Brownian clusters as a Markov process on the configuration space $\Omega$. For this, functions $d: \mathbb{N} \rightarrow(0, \infty)$, $\alpha: \mathbb{N} \times \mathbb{N} \rightarrow(0, \infty)$ and $\beta: \mathbb{N} \times \mathbb{N} \rightarrow(0, \infty)$ are given which represent the diffusion 
coefficient, the coagulation rate and the fragmentation rate respectively. We assume that both $\alpha$ and $\beta$ are symmetric. Also a parameter $\chi \in[0, \infty)$ and a continuously differentiable function $V: \mathbb{R}^{d} \rightarrow[0, \infty)$ are given for our model. We then define a Markov process $\omega(t)$ with infinitesimal generator $\mathcal{A}=\mathcal{A}_{0}+\mathcal{A}_{c}+\mathcal{A}_{f}$, where $\mathcal{A}_{0}$ represents the "Brownian motion" part of the dynamics, and $\mathcal{A}_{c}$ and $\mathcal{A}_{f}$ represent the coagulation and fragmentation parts of the evolution. For the "Brownian motion" part, we use the representation $\omega=\left(x_{i}, m_{i}\right)_{i \in I}$ to write

$$
\mathcal{A}_{0} F(\omega)=\sum_{i \in I} d\left(m_{i}\right) \Delta_{x_{i}} F(\omega),
$$

for any $C^{2}$ function $F$. Here $\Delta_{x_{i}}$ represents the Laplace operator which acts on the $x_{i}$ th variable.

As for the coagulation part, we write $\mathcal{A}_{c} F(\omega)=\mathcal{A}_{c}^{+} F(\omega)-\mathcal{A}_{c}^{-} F(\omega)$ with $\mathcal{A}_{c}^{+}$and $\mathcal{A}_{c}^{-}$given by

$$
\begin{aligned}
\mathcal{A}_{c}^{+} F(\omega)= & \frac{1}{2} \sum_{\substack{i, j \in I \\
i \neq j}} \alpha\left(m_{i}, m_{j}\right) V_{\varepsilon}\left(x_{i}-x_{j} ; m_{i}, m_{j}\right)\left[\frac{m_{i}}{m_{i}+m_{j}} F\left(S_{i j}^{1} \omega\right)\right. \\
& \left.+\frac{m_{j}}{m_{i}+m_{j}} F\left(S_{i j}^{2} \omega\right)\right] \\
\mathcal{A}_{c}^{-} F(\omega)= & \frac{1}{2} \sum_{\substack{i, j \in I \\
i \neq j}} \alpha\left(m_{i}, m_{j}\right) V_{\varepsilon}\left(x_{i}-x_{j} ; m_{i}, m_{j}\right) F(\omega) .
\end{aligned}
$$

Here,

(i) $\varepsilon>0$ is a small parameter that represents the range of interaction.

(ii) The function $V_{\varepsilon}(x ; m, n)=\lambda(\varepsilon) V\left(\frac{x}{\varepsilon} ; m, n\right)$, where

$$
\lambda(\varepsilon)= \begin{cases}|\log \varepsilon|^{-1} \varepsilon^{-2} & \text { if } d=2, \\ \varepsilon^{-2} & \text { if } d \geq 3,\end{cases}
$$

and

$$
V(x ; m, n)=r(m, n)^{-2} V\left(\frac{x}{r(m, n)}\right),
$$

with $r(m, n)=r(m)+r(n)$, for $r(n)=n^{\chi}$, and $V$ is a symmetric Hölder continuous function of compact support and total integral 1 .

(iii) We denote by $S_{i j}^{1} \omega$ the configuration formed from $\omega$ by removing $x_{j}$ from $\mathbf{x}$ and assigning the size $m_{i}+m_{j}$ to the surviving cluster at $x_{i}$. The configuration $S_{i j}^{2} \omega$ is defined in the same way, except that we remove $x_{i}$ from $\mathbf{x}$ and assign the size $m_{i}+m_{j}$ to the cluster at $x_{j}$. We note that the cluster at $x_{i}$ survives the coagulation with probability $\frac{m_{i}}{m_{i}+m_{j}}$.

Before describing the fragmentation part of the dynamics, let us explain the form of the function $V_{\varepsilon}$. Note that $V_{\varepsilon}\left(x_{i}-x_{j} ; m_{i}, m_{j}\right) \neq 0$ only if $x_{i}-x_{j}$ is of order $\varepsilon\left(r\left(m_{i}\right)+r\left(m_{j}\right)\right)$ with $r(m)=m^{\chi}$. This means that we regard a particle of size $m$ to be roughly a ball of radius $\varepsilon r(m)$ so that a pair of clusters of centers $x_{i}$ and $x_{j}$ coagulate 
when their corresponding balls overlap. If we assume that the mass of the $i^{\text {th }}$ cluster is distributed evenly in the ball $B_{r\left(m_{i}\right)}\left(x_{i}\right)$, then we expect to have $\chi=\frac{1}{d}$. However, in reality a cluster is far from being a round ball and expected to be a fractal like object. By allowing $\chi \in(0, \infty)$ we are hoping to have a more physically relevant model. In particular, the case $\chi<\frac{1}{d}$ represents a scenario in which the ball $B_{r\left(m_{i}\right)}\left(x_{i}\right)$ contains the true cluster and only a fraction of the ball is occupied with the cluster. We believe that the case $\chi>\frac{1}{d-2}$ corresponds to the occurrence of "gelation". We refer to [HR1,HR3 and R2] for more discussions. (Note that no finite $\chi$ can cause gelation when $d=2$; we guess that the radius must grow exponentially with the mass in order to have a gel in this case.)

The occurrence of the factor $\lambda(\varepsilon)$ in the definition of $V_{\varepsilon}$ is to guarantee that when two clusters collide, then they coagulate with a probability that stays away from 0 as $\varepsilon \rightarrow 0$. Indeed $B=x_{i}-x_{j}$ is a Brownian motion that spends a time of order $\varepsilon^{2} r\left(m_{i}, m_{j}\right)^{2}$ (respectively $\left.\varepsilon^{2} r\left(m_{i}, m_{j}\right)^{2}|\log \varepsilon|\right)$ in the support of $V_{\varepsilon}$ when $d \geq 3$ (respectively $d=2$ ). We also multiply the sum in the definition $\mathcal{A}_{c}$ by $1 / 2$ to ensure that the summation is over unordered pairs $\{i, j\}$.

As for the fragmentation part, $\mathcal{A}_{f} F(\omega)=\mathcal{A}_{f}^{+} F(\omega)-\mathcal{A}_{f}^{-} F(\omega)$, is given by

$$
\frac{1}{2} \sum_{i} \sum_{m=1}^{m_{i}-1} \beta\left(m, m_{i}-m\right) \int V^{\varepsilon}\left(x_{i}-y ; m_{i}-m, m\right)\left(F\left(S_{i}^{y, m} \omega\right)-F(\omega)\right) d y,
$$

with

$$
\mathcal{A}_{f}^{+} F(\omega)=\frac{1}{2} \sum_{i} \sum_{m=1}^{m_{i}-1} \beta\left(m, m_{i}-m\right) \int V^{\varepsilon}\left(x_{i}-y ; m_{i}-m, m\right) F\left(S_{i}^{y, m} \omega\right) d y .
$$

Here,

$$
V^{\varepsilon}(a ; m, n)=\varepsilon^{-d} V\left(\frac{a}{\varepsilon} ; m, n\right),
$$

and $S_{i}^{y, m}$ is that configuration formed from $\omega$ by replacing $\left(x_{i}, m_{i}\right)$ with a pair of clusters of positions $x_{i}$ and $y$ and sizes $m$ and $m_{i}-m$.

The central object to study is the cluster density of a given size. Microscopically we are interested in the empirical measures

$$
g_{n}^{\varepsilon}(d x, t)=K_{\varepsilon}^{-1} \sum_{i} \delta_{x_{i}(t)}(d x) 11\left(m_{i}(t)=n\right),
$$

where $K_{\varepsilon}$ was defined by (1.1). If for example we select $\left(x_{1}(0), m_{1}(0)\right), \ldots,\left(x_{N}(0)\right.$, $\left.m_{N}(0)\right)$ randomly and independently with the law

$$
\mathbb{P}\left(x_{i}(0) \in A, m_{i}(0)=n\right)=\frac{1}{Z} \int_{A} f_{n}^{0}(x) d x,
$$

with $Z=\sum_{n} \int f_{n}^{0} d x$, then by the law of large numbers, $g_{n}^{\varepsilon}(d x, 0)$ converges weakly to $f_{n}^{0}(x) d x$ provided that $N=K_{\varepsilon} Z$ and $\varepsilon \rightarrow 0$. Note that such a choice of initial condition implies that on average there are $K_{\varepsilon} \int f_{n}^{0}(x) d x$ many particles of size $n$. A Wiener Sausage calculation reveals that in average, each particle in our model experiences finitely many coagulations per unit time. This explains our reason for choosing 
$K_{\varepsilon}$ as above. The main result of [HR1,HR2 and R1] states that if $\chi<\frac{1}{d-2}$, there is no fragmentation, and $\alpha$ satisfies some technical conditions, then the empirical density $g_{n}^{\varepsilon}(d x, t)$ converges to $f_{n}(x, t) d x$ where $f_{n}$ is a solution to Smoluchowski's equation, subject to the initial condition $f_{n}(x, 0)=f_{n}^{0}(x)$. It is shown in [HR3] that this solution is unique.

Smoluchowski's equation has the form

$$
\frac{\partial f_{n}}{\partial t}(x, t)=d(n) \Delta_{x} f_{n}(x, t)+Q_{n}^{+, c}(\mathbf{f})-Q_{n}^{-, c}(\mathbf{f})+Q_{n}^{+, f}(\mathbf{f})-Q_{n}^{-, f}(\mathbf{f}),
$$

where $\mathbf{f}=\left(f_{n}: n \in \mathbb{N}\right)$, and

$$
\begin{aligned}
& Q_{n}^{+, c}(\mathbf{f})=\frac{1}{2} \sum_{m=1}^{n} \hat{\alpha}(m, n-m) f_{m} f_{n-m}, \quad Q_{n}^{-, c}(\mathbf{f})=\sum_{m=1}^{\infty} \hat{\alpha}(m, n) f_{m} f_{n}, \\
& Q_{n}^{+, f}(\mathbf{f})=\sum_{m=1}^{\infty} \hat{\beta}(m, n) f_{n+m}, \quad Q_{n}^{-, f}(\mathbf{f})=\frac{1}{2} \sum_{m=1}^{n-1} \hat{\beta}(m, n-m) f_{n},
\end{aligned}
$$

with

$$
\hat{\alpha}(m, n)=\eta(m, n) \alpha(m, n), \quad \hat{\beta}(m, n)=\eta(m, n) \beta(m, n) .
$$

The function $\eta(m, n)$ is calculated in terms of the microscopic details of the model. We start with the case $d=2$. In this case $\eta$ is independent of the function $V$ and the parameter $\chi$, and is simply given by

$$
\eta(m, n)=\frac{2 \pi(d(m)+d(n))}{2 \pi(d(m)+d(n))+\alpha(m, n)} .
$$

The formula for $\eta(m, n)$ is more complicated when $d \geq 3$ and does depend on both $V$ and $\chi$. Here is the recipe for $\eta$ : First we find the unique solution to the equation

$$
(d(m)+d(n)) \Delta u_{m, n}(x)=\alpha(m, n) V(x ; m, n)\left(1+u_{m, n}(x)\right)
$$

with $u(x ; m, n)=u_{m, n}(x)$ satisfying $u_{m, n}(x) \rightarrow 0$, as $|x| \rightarrow \infty$. Then we set

$$
\eta(m, n)=\int V(x ; m, n)\left(1+u_{m, n}(x)\right) d x .
$$

Remark 2.1. - For the purposes of this section, we have assumed that $\sum_{n} \int f_{n}^{0} d x<\infty$, which implies that there are finitely many particles almost surely. However in Sect. 3 when the main result of this article is discussed, the density $f_{n}^{0}$ is constant and the system involves infinitely many particles. The existence of such a particle system is no longer obvious, and in Remark 3.5 we will explain how such a particle system is constructed.

- Note that we deliberately choose a mechanism for the fragmentation that is, in some sense, dual to the coagulation mechanism. This allows us to easily construct reversible invariant measures for the process $\omega(t)$. In other words the fragmentation is defined in such a way that if we reverse time after a coagulation, we obtain a fragmentation. For the kinetic limit however, we can use a kernel $W$ for the fragmentation that is different from $V$, or even choose two new locations $y_{1}$ and $y_{2}$ near $x_{i}$ for the locations of new clusters of a fragmented cluster. However, for this fragmentation mechanism, the macroscopic coagulation and fragmentation rates read $\hat{\alpha}=\alpha \eta, \hat{\beta}=\beta \eta^{\prime}$ with possibly $\eta \neq \eta^{\prime}$. 
- Let us write $Q_{n}=Q_{n}^{+, c}-Q_{n}^{-, c}+Q_{n}^{+, f}-Q_{n}^{-, f}$. We then have the following useful formula: For any sequence $\left(J_{n}: n \in \mathbb{N}\right)$,

$$
\sum_{n} J_{n} Q_{n}=\frac{1}{2} \sum_{n, m}\left(\hat{\alpha}(m, n) f_{m} f_{n}-\hat{\beta}(m, n) f_{m+n}\right)\left(J_{m+n}-J_{m}-J_{n}\right) .
$$

The main goal of this article is to derive an equation for the evolution of the density fluctuations about the solution to Smoluchowski's equation.

To this end, recall the fluctuation fields $\xi_{n}^{\varepsilon}(d x, t)$ that was defined by (1.2). Let us assume that $\chi<(d-2)^{-1}$ and that the total mass $\int \sum_{n} n f_{n}^{0} d x$ is finite.

Conjecture 2.1. As $\varepsilon \rightarrow 0$, the process $\xi_{n}^{\varepsilon}$ converges to $\xi_{n}$, where $\xi_{n}$ is the unique solution to the Uhlenbeck-Ornstein equation

$$
\begin{aligned}
\frac{\partial \xi_{n}}{\partial t} & =d(n) \Delta_{x} \xi_{n}+\mathcal{L}_{n}^{c} \boldsymbol{\xi}+\mathcal{L}_{n}^{f} \boldsymbol{\xi}+\gamma_{n}, \\
\xi_{n}(x, 0) & =\bar{\xi}_{n}(x),
\end{aligned}
$$

where $\boldsymbol{\xi}=\left(\xi_{n}: n \in \mathbb{N}\right)$, and

$$
\mathcal{L}_{n}^{c}=\mathcal{L}_{n}^{+, c}-\mathcal{L}_{n}^{-, c}, \quad \mathcal{L}_{n}^{f}=\mathcal{L}_{n}^{+, f}-\mathcal{L}_{n}^{-, f},
$$

with

$$
\begin{aligned}
\mathcal{L}_{n}^{+, c} \boldsymbol{\xi} & =\sum_{m=1}^{n-1} \hat{\alpha}(m, n-m) f_{m} \xi_{n-m}, \quad \mathcal{L}_{n}^{-, c} \boldsymbol{\xi}=2 \sum_{m=1}^{\infty} \hat{\alpha}(m, n)\left(f_{m} \xi_{n}+f_{n} \xi_{m}\right), \\
\mathcal{L}_{n}^{+, f} \boldsymbol{\xi} & =\sum_{m=1}^{\infty} \hat{\beta}(m, n) \xi_{n+m}, \quad \mathcal{L}_{n}^{-, f} \boldsymbol{\xi}=\frac{1}{2} \sum_{m=1}^{n-1} \hat{\beta}(m, n-m) \xi_{n},
\end{aligned}
$$

and $\gamma_{n}$ is a space-time white noise with variance given by

$$
\begin{aligned}
\left\langle\left(\sum_{n} \iint J_{n} \gamma_{n} d x d t\right)^{2}\right\rangle= & 2 \iint \sum_{n} d(n) f_{n}\left|\nabla J_{n}\right|^{2} d x d t \\
& +\frac{1}{2} \iint \sum_{m, n} \hat{\alpha}(m, n) f_{n} f_{m}\left(J_{n+m}-J_{n}-J_{m}\right)^{2} d x d t \\
& +\frac{1}{2} \iint \sum_{m, n} \hat{\beta}(m, n) f_{n+m}\left(J_{n+m}-J_{n}-J_{m}\right)^{2} d x d t
\end{aligned}
$$

for any smooth test function $J=\left(J_{n}: n \in \mathbb{N}\right)$ of compact support in $\mathbb{R}^{d} \times(0, \infty)$.

In fact $\gamma$ belongs to a suitable negative Sobolev space and the integral of $J_{n} \gamma_{n}$ must be understood as the value of the distribution $\gamma_{n}$ at the smooth test function $J_{n}$. See the next section or the beginning of Sect. 8 for the precise definition of $\xi$ and $\gamma$ and the meaning of Eq. (2.11).

The main result of this paper asserts that Conjecture 2.1 is valid if the initial distribution of the cluster is chosen according to a reversible equilibrium state and $d=2$. 


\section{Equilibrium Fluctuations}

We start with constructing reversible invariant measures for the process $\omega(t)$. For this we take a collection of positive numbers $\lambda=\left(\lambda_{n}: n \in \mathbb{N}\right)$ such that $\sum_{n} \lambda_{n}<\infty$, and

$$
\alpha(m, n) \lambda_{n} \lambda_{m}=\beta(m, n) \lambda_{n+m}
$$

for every $m, n \in \mathbb{N}$. Note that for such a collection, the functions $f_{n}(x, t) \equiv \lambda_{n}$ do solve Smoluchowski's equation because by (2.7) and (3.1),

$$
\hat{\alpha}(m, n) \lambda_{n} \lambda_{m}=\hat{\beta}(m, n) \lambda_{n+m},
$$

and this in turn implies

$$
Q_{n}^{+, c}(\boldsymbol{\lambda})=Q_{n}^{-, f}(\boldsymbol{\lambda}), \quad Q_{n}^{-, c}(\boldsymbol{\lambda})=Q_{n}^{+, f}(\boldsymbol{\lambda}) .
$$

Given such $\lambda$, we construct a reversible invariant measure $\mu_{\lambda}$ for our process $\omega(t)$ : Let $\mathbf{x}^{n}$ be a Poisson point process with intensity $K_{\varepsilon} \lambda_{n}$. Assume that $\left(\mathbf{x}^{n}, n \in \mathbb{N}\right)$ are independent and define $\omega=(\mathbf{x}, \mathbf{m})$ by $\mathbf{x}=\bigcup_{n=1}^{\infty} \mathbf{x}^{n}$ and $\mathbf{m}(a)=n$ for $a \in \mathbf{x}^{n}$. In words, particles of size $n$ form a Poisson point process of intensity of $K_{\varepsilon} \lambda_{n}$ and these processes are independent for different choices of $n$. We note that if $\Lambda$ is a bounded subset of $\mathbb{R}^{d}$, then

$$
\int M_{\Lambda} d \mu_{\lambda}=|\Lambda| K_{\varepsilon} \sum_{n} \lambda_{n}
$$

where

$$
M_{\Lambda}^{n}(\omega)=M_{\Lambda}^{n}(\mathbf{x}, \mathbf{m})=\#\{a \in \mathbf{x}: a \in \Lambda, \mathbf{m}(a)=n\}, \quad M_{\Lambda}=\sum_{n=1}^{\infty} M_{\Lambda}^{n} .
$$

Hence, if we assume that $\sum_{n} \lambda_{n}<\infty$, then there are finitely many clusters in a bounded domain almost surely with respect to $\mu_{\lambda}$.

We now assert that $\mu_{\lambda}$ is indeed reversible. To explain this, let us take two bounded local $C^{2}$ functions $F, G: \Omega \rightarrow \mathbb{R}$. By a local function $F$ we mean that there exists a positive constant $c_{0}$ such that $F$ depends only on particles $\left(x_{i}, m_{i}\right)$ such that $\left|x_{i}\right|, m_{i} \leq c_{0}$. We then have

$$
\int G \mathcal{A} F d \mu_{\lambda}=\int F \mathcal{A} G d \mu_{\lambda}
$$

Indeed,

$$
\begin{aligned}
& \int G \mathcal{A}_{0} F d \mu_{\lambda}=-\int \sum_{i} d\left(m_{i}\right) \nabla_{x_{i}} F \cdot \nabla_{x_{i}} G d \mu_{\lambda}, \\
& \int G \mathcal{A}_{c}^{+} F d \mu_{\lambda}=\int F \mathcal{A}_{f}^{-} G d \mu_{\lambda}, \quad \int G \mathcal{A}_{c}^{-} F d \mu_{\lambda}=\int F \mathcal{A}_{f}^{+} G d \mu_{\lambda} .
\end{aligned}
$$

Note that (3.6) is the microscopic analog of (3.3), and together with (3.5) imply (3.4). The proof of (3.5) follows from an integration by parts. As for (3.6), observe that for any bounded set $\Lambda$,

$$
\mu_{\lambda}^{\Lambda}\left(d \omega_{\Lambda}\right)=\sum_{L_{1}, L_{2}, \ldots} \prod_{n=1}^{\infty}\left[\prod_{i=1}^{L_{n}} \mathbb{1}\left(m_{n i}=n, x_{n i} \in \Lambda\right) d x_{n i}\right] \frac{\left(\lambda_{n} K_{\varepsilon}\right)^{L_{n}}}{L_{n} !} e^{-\lambda_{n} K_{\varepsilon}|\Lambda|},
$$


where $\omega_{\Lambda}$ is the configuration in the set $\Lambda$ and $\mu_{\lambda}^{\Lambda}$ is the law of $\omega_{\Lambda}$ under $\mu_{\lambda}$. Here we have labeled particles of size $n$ by $n 1, n 2, \ldots, n L_{n}$ and $L_{n}=M_{\Lambda}^{n}$ is the number of such particles. Using the representation (3.7), one can readily verify (3.6).

Let us write $\mathbb{P}_{\varepsilon}^{\omega}$ and $\mathbb{E}_{\varepsilon}^{\omega}$ for the probability and the expectation with respect to the process $\omega(\cdot)$ subject to the initial condition $\omega(0)=\omega$. When $\omega(0)$ is distributed according to an invariant measure $\mu_{\lambda}$, we write $\mathbb{P}_{\varepsilon}^{e q}$ and $\mathbb{E}_{\varepsilon}^{e q}$ instead. Given $\omega(\cdot)$, we define

$$
\xi_{n}^{\varepsilon}(t, J)=\sqrt{K_{\varepsilon}}\left(\frac{1}{K_{\varepsilon}} \sum_{i} J\left(x_{i}(t)\right) \mathbb{1}\left(m_{i}(t)=n\right)-\lambda_{n} \int J(x) d x\right)
$$

for every smooth $J: \mathbb{R}^{d} \rightarrow \mathbb{R}$ of compact support. Let $\mathcal{D}$ denote the space of smooth functions of compact support and let $\mathcal{D}^{\prime}$ denote the space of distributions (the dual of $\mathcal{D})$. We regard $\xi_{n}^{\varepsilon}$ as an element of the Skorohod space $\mathbb{D}=D\left([0, T],\left(\mathcal{D}^{\prime}\right)^{\mathbb{N}}\right)$. The transformation $\omega(\cdot) \mapsto \xi^{\varepsilon}$ induces a probability measure $\mathcal{P}^{\varepsilon}$ on $\mathbb{D}$. We regard $\xi_{n}^{\varepsilon}(t, J)$ as the value of the distribution $\xi_{n}^{\varepsilon}(t)$ at $J$.

To state our assumptions, take a nondecreasing function $a \geq 1$, such that $\alpha^{\prime}(m, n)=$ $\alpha(m, n) /(d(m)+d(n)) \leq a(n)+a(m)$, and set $\beta^{\prime}(n)=\sum_{m=1}^{n-1} \beta(n-m, m)$.

Hypothesis 3.1. The function $d(\cdot)$ is bounded. Moreover for some $\theta>1 / 2$,

$$
\lim _{\varepsilon \rightarrow 0} \tau(\varepsilon):=\lim _{\varepsilon \rightarrow 0} K_{\varepsilon}^{1 / 2} \sum_{2 \varepsilon r(n)>\delta(\varepsilon)} a(n) \lambda_{n}=0,
$$

where $\delta(\varepsilon)=|\log \varepsilon|^{-\theta}$, and

$$
\sum_{n}\left[a(n)\left(r(n)+\beta^{\prime}(n) \log n\right)+a(n)^{2}(a(n)+\log n)\right] \lambda_{n}<\infty .
$$

Remark 3.1. Note that by detailed balance, we have that $\beta(n, m)=\alpha(m, n) \lambda_{n} \lambda_{m} / \lambda_{m+n}$. Hence, if $\alpha$ and $\lambda$ are known, then $\beta$ is determined. As an example, consider the case with $\lambda_{n}$ decaying like $e^{-c n}$, as $n \rightarrow \infty$. In this case, we can readily see that if $a(n)$ is growing at most like a polynomial as $n$ gets large, then both (3.9) and (3.10) are satisfied.

Theorem 3.1. Assume Hypothesis 3.1 and that the dimension $d=2$. Then the finite dimensional marginals of the sequence $\mathcal{P}^{\varepsilon}$ converges to the finite dimensional marginals of $\mathcal{P}$, where $\mathcal{P}$ is the distribution of a stationary Ornstein-Uhlenbeck Gaussian process with covariance

$$
\iint \sum_{n=1}^{\infty} \xi_{n}\left(t, J_{n}\right) \xi_{n}\left(0, H_{n}\right) \mathcal{P}(d \xi)=\int \sum_{n=1}^{\infty}\left(T_{t} J_{n}\right)(x) H_{n}(x) \lambda_{n} d x .
$$

Here $J_{n}, H_{n} \in \mathcal{D}$ for $n \in \mathbb{N}$ and $T_{t}$ is the semigroup generated by the linear Smoluchowski's operator

$$
\begin{aligned}
(\Gamma \mathbf{J})_{n}=d & (n) \Delta_{x} J_{n}+\sum_{m=1}^{\infty} \hat{\beta}(m, n) J_{n+m}-\frac{1}{2} \sum_{m=1}^{n-1} \hat{\beta}(m, n-m) J_{n} \\
& +\sum_{m=1}^{n-1} \hat{\alpha}(m, n-m) J_{n-m} \lambda_{m}-\sum_{m=1}^{\infty} \hat{\alpha}(m, n)\left(J_{n} \lambda_{m}+J_{m} \lambda_{n}\right) .
\end{aligned}
$$


Remark 3.2. Note that the macroscopic coagulation and fragmentation rates $\hat{\alpha}$ and $\hat{\beta}$ are strictly smaller than their microscopic counterparts $\alpha$ and $\beta$. We refer the reader to Sect. 4 for a heuristic explanation and how a fundamental auxiliary function $u^{\varepsilon}$ would allow us to switch from the microscopic rates $\alpha$ and $\beta$ to macroscopic rates $\hat{\alpha}$ and $\hat{\beta}$. Note also that even though the "strengths" of the noises associated with the coagulation and fragmentation are given by $\alpha$ and $\beta$, the corresponding macroscopic "strengths" are given by $\hat{\alpha}$ and $\hat{\beta}$ as the expressions (2.15) and (2.15) indicate. In fact this reduction in the strength happens in a very curious way:

- The auxiliary function $u^{\varepsilon}$ corrects the original noises coming from the coagulation and fragmention by reducing their strengths to $\tilde{\alpha}=\alpha \eta^{2}$ and $\tilde{\beta}=\beta \eta^{2}$. (See formulas (8.33) and (8.37) and the definitions of $A_{c 0}$ and $A_{f 0}$ which are given right after (8.26) and (8.34).)

- The Brownian part of the dynamics uses the corrector $u^{\varepsilon}$ and produces some noise which enhances the reduced strengths $\tilde{\alpha}$ and $\tilde{\beta}$ to their final values $\hat{\alpha}$ and $\hat{\beta}$. (See formula (8.24), expression $A_{02121111}$ which is defined right before (8.23), and the final step of the proof of (8.4).)

Remark 3.3. In fact what we can prove is somewhat stronger than what has appeared in the statement of Theorem 3.1. We will show that the process $\xi^{\varepsilon}=\xi^{\prime}-\xi^{\prime \prime}$ with both $\xi^{\prime}$ and $\xi^{\prime \prime}$ stationary processes in time, where the law of $\xi^{\prime}$ under $\mathbb{P}_{\varepsilon}^{e q}$ converges to $\mathcal{P}$, and

$$
\lim _{\varepsilon \rightarrow 0} \mathbb{E}_{\varepsilon}^{e q}\left|\xi^{\prime \prime}(t, J)\right|=0
$$

for every $t, n \in \mathbb{N}$, and test function $J$. We refer the reader to Sect. 9 for the details.

An alternative description of the law $\mathcal{P}$ is the martingale formulation of Holley and Stroock [HS] that will be defined in Sect. 8. It is this formulation which we use for the proof of Theorem 3.1 .

Remark 3.4. The intuition behind (3.11) is the standard dissipation-fluctuation principle. This principle is used to predict the form of the diffusion coefficient once the drift and the invariant measure for the fluctuation fields are known. In fact (3.11) is equivalent to saying that the process $\xi$ is a solution to the stochastic differential equation

$$
d \xi=\Gamma \xi d t+\mathcal{B} d \mathbf{W}_{t},
$$

where $d \mathbf{W}_{t}=\left(d W_{1, t}, \ldots, d W_{n, t}, \ldots\right)$ with $\left(d W_{n}: n \in \mathbb{N}\right)$ independent space-time white noises and the operator $\mathcal{B}$ is determined by

$$
\begin{aligned}
& \int \sum_{n=1}^{\infty}(\mathcal{B} \zeta)_{n}(\mathcal{B} \mathbf{H})_{n} d x=2 \int \sum_{n=1}^{\infty} \lambda_{n} \nabla_{x} J_{n} \cdot \nabla_{x} H_{n} d x \\
& +\frac{1}{2} \int \sum_{m, n=1}^{\infty} \hat{\alpha}(m, n) \lambda_{n} \lambda_{m}\left(J_{n+m}-J_{n}-J_{m}\right)\left(H_{n+m}-H_{n}-H_{m}\right) d x \\
& +\frac{1}{2} \int \sum_{m, n=1}^{\infty} \hat{\beta}(m, n) \lambda_{n+m}\left(J_{n+m}-J_{n}-J_{m}\right)\left(H_{n+m}-H_{n}-H_{m}\right) d x .
\end{aligned}
$$


Indeed if we start with the ansatz that $\xi$ satisfies an Ornstein-Uhlenbeck equation of the form (3.13), then we have an obvious guess for the linear drift $\Gamma \xi$, namely the linearization of the right-hand side of the macroscopic equation (2.6). We also have a candidate for its invariant measure, namely the measure $\mathcal{P}^{0}$ given by (3.11) at $t=0$;

$$
\iint \sum_{n=1}^{\infty} \xi_{n}^{0}\left(J_{n}\right) \xi_{n}^{0}\left(H_{n}\right) \mathcal{P}^{0}\left(d \xi^{0}\right)=\int \sum_{n=1}^{\infty} J_{n}(x) H_{n}(x) \lambda_{n} d x .
$$

We then select the diffusion operator $\mathcal{B}$ to be compatible with what we have for the drift and the invariant measure of the process $\xi$.

Remark 3.5. As our final remark, we comment that it is not obvious that our Markov process $\omega(\cdot)$ exists because we are dealing with infinitely many interacting diffusions. However, since we are only interested in the process $\omega(\cdot)$ at equilibrium, its existence can be shown by rather standard arguments which we now sketch.

(i) Observe that if initial macroscopic densities $\left(f_{n}^{0}: n \in \mathbb{N}\right)$ satisfy $\sum_{n} \int f_{n}^{0}$ $d x<\infty$, then we can construct our process by starting from $N$ independent particles $\left(x_{1}, m_{1}\right), \ldots,\left(x_{N}, m_{N}\right)$ satisfying (2.5), where $N$ and $\varepsilon$ are related by the equation $N=K_{\varepsilon} \sum_{n} \int f_{n}^{0} d x$. In other words, if the total density is finite macroscopically, then initially we are dealing with finitely many particles almost surely and the existence of the process $\omega(\cdot)$ is obvious. However, since at equilibrium $f_{n}^{0} \equiv \lambda_{n}$ is not integrable, we need to consider a Poisson point process with infinitely many particles.

(ii) We now argue that we can construct our process if we make two assumptions:

$$
\begin{aligned}
& \sum_{n} \int_{|x| \leq r} n f_{n}^{0}(x) d x<\infty, \\
& \alpha(m, n)=\beta(m, n)=0 \text { if } m+n>\ell,
\end{aligned}
$$

for every $r>0$ and some $\ell>0$. In other words, we assume that locally the total mass is finite macroscopically but now we assume that no interaction occurs if particles are large. To construct $\omega(\cdot)$ in this case, we first replace $f^{0}$ with $f^{0} 11(|x| \leq k)$. Our process exists for such an initial macroscopic density by $(i)$. The corresponding process is denoted by $\omega_{k}(\cdot)$. We now want to send $k$ to infinity and show that the sequence $\left(\omega_{k}: k \in \mathbb{N}\right)$ is tight and that any of its limit point $\omega$ is a solution to the martingale problem associated with the generator $\mathcal{A}$. That is, $F(\omega(t))-\int_{0}^{t} \mathcal{A} F\left(\omega(s) d s\right.$, is a martingale for every $C^{2}$ local function $F$. This can be readily achieved by establishing a control on the total number of particles in a ball $\{x:|x| \leq r\}$. Here is a way of establishing such a control uniformly in $k$ : Pick a positive smooth function $J$ which equals to $\exp (-|x|)$ for large $x$, and set $H(x)=-\int_{|y| \leq 1} \log |y| J(x-y) d y$. We can readily show that $H>0$ and that $\Delta H \leq c_{0} H$ for a constant $c_{0}$. Then use the martingale $M(t)=F\left(\omega_{k}(t)\right)-\int_{0}^{t} \mathcal{A} F\left(\omega_{k}(s) d s\right.$ for $F(\omega)=\sum_{i} H\left(x_{i}\right) m_{i}$ to show

$$
\sup _{k} \mathbb{E} \sup _{t \in[0, T]} F\left(\omega_{k}(t)\right)^{2}<\infty,
$$

for every $T$. This can be used to establish the tightness of $\omega_{k}$ and the existence of our process provided that (3.14) and (3.15) are true. 
(iii) It remains to relax the restriction (3.15). We now would like to take advantage of the fact that we only need to consider $f_{n}^{0} \equiv \lambda_{n}$. More precisely, by $(i i)$, we know that $\mathbb{P}^{e q}$ exists if we assume that (3.15) is true. Let us write $\omega^{\ell}$ for our process when $\alpha$ and $\beta$ are replaced with $\alpha_{\ell}(m, n)=\alpha(m, n) 11(m+n \leq \ell)$, $\left.\beta_{\ell}(m, n)=\beta(m, n) \mathbb{1 1}(m+n) \leq \ell\right)$. Again, we need to show the tightness of $\omega^{\ell}$ and pass to the limit in the martingale formulation of our process. For this, we need to show something like (3.16) for the sequence $\omega^{\ell}$. This can be readily achieved by bounding various terms that appear in the martingale $M(\cdot)$, using the fact that the process $\omega^{\ell}$ is stationary in time.

\section{A Sketch of the Proof}

We aim to show that the expression

$$
X_{\varepsilon}(\omega(t))=K_{\varepsilon}^{-1 / 2} \sum_{i} J\left(x_{i}(t), m_{i}(t)\right)
$$

with $J(x, n)=J_{n}(x)$ satisfying $\int J_{n} d x=0$, is close to $\sum_{n} \xi_{n}\left(t, J_{n}\right)$, with the distributions $\left(\xi_{n}: n \in \mathbb{N}\right)$ solving (3.13) in the weak sense. To derive (3.13), we use Markov property of the process $\omega(t)$ to write

$$
\begin{aligned}
X_{\varepsilon}(\omega(t))= & X_{\varepsilon}(\omega(0))+\sum_{n} \int_{0}^{t} \mathcal{A}_{0} X_{\varepsilon}(\omega(s)) d s+\int_{0}^{t} \mathcal{A}_{c} X_{\varepsilon}(\omega(s)) d s \\
& +\int_{0}^{t} \mathcal{A}_{f} X_{\varepsilon}(\omega(s)) d s+M_{\varepsilon}(t) \\
= & : Y_{\varepsilon}^{1}+Y_{\varepsilon}^{2}(t)+Y_{\varepsilon}^{3}(t)+Y_{\varepsilon}^{4}(t)+M_{\varepsilon}(t),
\end{aligned}
$$

with $M_{\varepsilon}$ a martingale for which

$$
N_{\varepsilon}(t)=M_{\varepsilon}(t)^{2}-\int_{0}^{t}\left(\mathcal{A} X_{\varepsilon}^{2}-2 X_{\varepsilon} \mathcal{A} X_{\varepsilon}\right)(\omega(s)) d s,
$$

is a martingale.

The identity (4.2) should be compared to what we have as the weak form of (3.13), namely

$$
\begin{aligned}
\sum_{n} \xi_{n}\left(J_{n}, t\right)= & \sum_{n} \xi_{n}\left(J_{n}, 0\right)+\int_{0}^{t} \sum_{n} d(n) \xi_{n}\left(\Delta J_{n}, s\right) d s \\
& +\int_{0}^{t} \sum_{m, n} \hat{\alpha}(m, n) \lambda_{m} \xi_{n}\left(J_{n+m}-J_{m}-J_{n}, s\right) d s \\
& +\int_{0}^{t} \sum_{m, n} \hat{\beta}(m, n) \xi_{n+m}\left(J_{n+m}-J_{m}-J_{n}, s\right) d s+M(t) \\
= & : Y^{1}+Y^{2}(t)+Y^{3}(t)+Y^{4}(t)+M(t),
\end{aligned}
$$


where the process $M(t)$ is a martingale for which

$$
\begin{aligned}
N(t)= & M(t)^{2}-t \int 2 \sum_{n} d(n) \lambda_{n}\left|\nabla J_{n}(x)\right|^{2} d x \\
& -\frac{t}{2} \int \sum_{m, n} \hat{\alpha}(m, n) \lambda_{m} \lambda_{n}\left(J_{n+m}-J_{n}-J_{m}\right)^{2}(x) d x \\
& -\frac{t}{2} \int \sum_{m, n} \hat{\beta}(m, n) \lambda_{n+m}\left(J_{n+m}-J_{n}-J_{m}\right)^{2}(x) d x,
\end{aligned}
$$

is a martingale.

To establish Theorem 3.1, we may try to show

$$
Y_{\varepsilon}^{j} \rightarrow Y^{j}, \quad M_{\varepsilon} \rightarrow M
$$

for $i=1, \ldots, 4$. It turns out that this is not what is going on! Firstly, it is rather straightforward to show that $Y_{\varepsilon}^{1} \rightarrow Y^{1}$ by the classical central limit theorem with $Y^{1}$ a Gaussian random variable with variance $\sum_{n} \lambda_{n} \int J_{n}^{2} d x$. Also, virtually by definition, we have that if $\boldsymbol{\xi}^{\varepsilon}$ converges to $\boldsymbol{\xi}$, then $Y_{\varepsilon}^{2} \rightarrow \sum_{n} d(n) \int_{0}^{t} \xi_{n}\left(\Delta J_{n}, s\right) d s$. This stems from the fact that $Y_{\varepsilon}^{2}$ corresponds to the "non-interacting" part of the evolution, namely the Laplacian operator $\Delta$. However we need to split the "interacting" part of the microscopic evolution into 3 distinct parts of completely different natures. Indeed, we have a decomposition

$$
Y_{\varepsilon}^{3}=Y_{\varepsilon}^{3,1}+Y_{\varepsilon}^{3,2}+Y_{\varepsilon}^{3,3},
$$

where $Y_{\varepsilon}^{3,1} \rightarrow Y^{3}$ as $\varepsilon \rightarrow 0$, the term $Y_{\varepsilon}^{3,2}$ contributes to the fragmentation term so that $Y_{\varepsilon}^{3,2}+Y_{\varepsilon}^{4} \rightarrow Y^{4}$, and $Y_{\varepsilon}^{3,3}$ contributes to the martingale part. That is, $Y_{\varepsilon}^{3,3}+M_{\varepsilon} \rightarrow M$. It is as if a part of the microscopic "drift" becomes some type of "white noise" as $\varepsilon \rightarrow 0$. Perhaps this is the most surprising aspect of the present work and is in complete contrast with some earlier works on the equilibrium and non-equilibrium fluctuations on models with diffusive scaling [CY,C] and a stochastic model with kinetic scaling [R1]. This ramification of the diffusion coefficient by the "drift" is reminiscent of a similar phenomenon for the tagged particles in the exclusion processes (see Kipnis-Vardhan [KV]). In our setting however, the ramification of the noise happens in a rather curious way as we explained in Remark 3.2.

To explain the decomposition (4.5), and sketch our method of proof further, we need to recall how Smoluchowski's equation has been derived from our microscopic model in the articles [HR1,HR2,R2 and HRY]. For this derivation, we need to understand how the microscopic coagulation (respectively fragmentation) rate $\alpha(m, n)$ (respectively $\beta(m, n)$ ) leads to the macroscopic coagulation rate $\hat{\alpha}(m, n)$ (respectively $\hat{\beta}(m, n)$ ).

For the derivation of (2.6), we start from the expression

$$
\hat{X}_{\varepsilon}(\omega(t))=K_{\varepsilon}^{-1 / 2} X_{\varepsilon}(\omega(t))=K_{\varepsilon}^{-1} \sum_{i} J\left(x_{i}(t), m_{i}(t)\right),
$$

and study the corresponding (4.2) which we obtain by multiplying both sides of (4.2) by $K_{\varepsilon}^{-1 / 2}$. Since $K_{\varepsilon}^{-1 / 2} M_{\varepsilon} \rightarrow 0$, we only need to concentrate on $K_{\varepsilon}^{-1 / 2} Y_{\varepsilon}^{3}$ and $K_{\varepsilon}^{-1 / 2} Y_{\varepsilon}^{4}$. The term $K_{\varepsilon}^{-1 / 2} Y_{\varepsilon}^{4}$ is in some sense linear and all challenges come from $K_{\varepsilon}^{-1 / 2} Y_{\varepsilon}^{3}$. It turns out that there is a splitting $K_{\varepsilon}^{-1 / 2} Y_{\varepsilon}^{3}=Z_{\varepsilon}^{1}+Z_{\varepsilon}^{2}$ with 
$Z_{\varepsilon}^{1}$ converging to $\int_{0}^{t} \int \sum_{n} J_{n}(x) Q_{n}^{c}(\mathbf{f})(x, t) d x$ and $Z_{\varepsilon}^{2}+K_{\varepsilon}^{-1 / 2} Y_{\varepsilon}^{4}$ converging to $\int_{0}^{t} \int \sum_{n} J_{n}(x) Q_{n}^{f}(\mathbf{f})(x, t) d x$. This splitting is not hard to justify; when a fragmentation occurs, a pair of particles are produced which are within a distance of order $O(\varepsilon)$ and prone to coagulate. Of course such a coagulation undoes the fragmentation that has just been occurred. Indeed $Z_{\varepsilon}^{2}$ is negative which results in a macroscopic fragmentation $\hat{\beta}$ strictly less than $\beta$.

To describe the decomposition (4.5), let us observe

$$
\begin{aligned}
Y_{\varepsilon}^{3} & =\frac{1}{2} K_{\varepsilon}^{-1 / 2} \sum_{i, j} \alpha\left(m_{i}, m_{j}\right) V_{\varepsilon}\left(x_{i}-x_{j} ; m_{i}, m_{j}\right) \tilde{J}\left(x_{i}, m_{i}, x_{j}, m_{j}\right) \\
& =\frac{1}{2} K_{\varepsilon}^{-3 / 2} \sum_{i, j} \alpha\left(m_{i}, m_{j}\right) V^{\varepsilon}\left(x_{i}-x_{j} ; m_{i}, m_{j}\right) \tilde{J}\left(x_{i}, m_{i}, x_{j}, m_{j}\right),
\end{aligned}
$$

where $V^{\varepsilon}=K_{\varepsilon} V_{\varepsilon}$ and $\tilde{J}\left(x_{i}, m_{i}, x_{j}, m_{j}\right)$ is given by

$$
\frac{m_{i}}{m_{i}+m_{j}} J\left(x_{i}, m_{i}+m_{j}\right)+\frac{m_{j}}{m_{i}+m_{j}} J\left(x_{j}, m_{i}+m_{j}\right)-J\left(x_{i}, m_{i}\right)-J\left(x_{j}, m_{j}\right) \text {. }
$$

Our goal would be a decomposition of the form

$$
\int_{0}^{t} Y_{\varepsilon}^{3}(s) d s=\int_{0}^{t} B_{\varepsilon}^{z}(\omega(s)) d s+\int_{0}^{t} C_{\varepsilon}(\omega(s)) d s+D_{\varepsilon}(t)+\text { Error },
$$

where

$$
B_{\varepsilon}^{z}(\omega)=\frac{1}{2} K_{\varepsilon}^{-3 / 2} \sum_{i, j} \alpha\left(m_{i}, m_{j}\right) W^{\varepsilon}\left(x_{i}-x_{j}+z ; m, n\right) \tilde{J}\left(x_{i}, m_{i}, x_{j}, m_{j}\right),
$$

for a suitable function $W^{\varepsilon}$ which will be defined shortly, and Error represents a term that will go to zero as $\varepsilon \rightarrow 0$ and $|z| \rightarrow 0$. The form of $W^{\varepsilon}$ would allow us to replace $\alpha$ with its macroscopic counterpart $\hat{\alpha}$. The term $C_{\varepsilon}$ is given by

$$
\begin{gathered}
\int K_{\varepsilon}^{-3 / 2} \sum_{i} \sum_{m=1}^{m_{i}-1} \beta\left(m, m_{i}-m\right) V^{\varepsilon}\left(x_{i}-y ; m, m_{i}-m\right) \\
u^{\varepsilon}\left(x_{i}-y ; m, m_{i}-m\right) \tilde{J}\left(x_{i}, m, y, m_{i}-m\right) d y
\end{gathered}
$$

and the term $D_{\varepsilon}(t)$ is a martingale. It is the decomposition (4.8) that leads to the decomposition (4.5).

To achieve the decomposition (4.8), fix $z$ and start from the expression

$$
G_{\varepsilon}(\omega)=K_{\varepsilon}^{-3 / 2} \sum_{i, j} \hat{u}^{\varepsilon}\left(x_{i}-x_{j} ; m_{i}, m_{j}\right) \tilde{J}\left(x_{i}, m_{i}, x_{j}, m_{j}\right),
$$

where $\hat{u}^{\varepsilon}(a ; m, n)=u^{\varepsilon}(a+z ; m, n)-u^{\varepsilon}(a ; m, n)$, with $u^{\varepsilon}(a ; m, n)$ satisfying the equation

$$
(d(m)+d(n)) \Delta u^{\varepsilon}(x ; m, n)=\alpha(m, n)\left[V_{\varepsilon}(x ; m, n) u^{\varepsilon}(x ; m, n)+V^{\varepsilon}(x ; m, n)\right] .
$$


(The functions $V^{\varepsilon}$ and $V_{\varepsilon}$ were defined by (2.4) and right before (2.2) respectively.) We then apply the martingale decomposition as in (4.2) to assert

$$
G_{\varepsilon}(\omega(t))=G_{\varepsilon}(\omega(0))+\int_{0}^{t} \mathcal{A} G_{\varepsilon}(\omega(s))+E_{\varepsilon}(t),
$$

with $E_{\varepsilon}(t)$ a martingale. This involves various terms as we apply the operators $\mathcal{A}_{0}, \mathcal{A}_{c}$ and $\mathcal{A}_{f}$ on $G_{\varepsilon}$. As it turns out, the first term $G_{\varepsilon}(\omega(0))$ and many other terms on the right-hand side of (4.12) are small if $|z|$ is sufficiently small. However, the choice of $u^{\varepsilon}$ results in a component in $\left(\mathcal{A}_{0}+\mathcal{A}_{c}\right) G_{\varepsilon}$, which is exactly our $2\left(B_{\varepsilon}^{z}-Y_{\varepsilon}^{3}\right)$ in (4.9), and a component in $\mathcal{A}_{f} G_{\varepsilon}$ which is exactly $C_{\varepsilon}$. The function $W^{\varepsilon}$ in (4.9) is given by

$$
W^{\varepsilon}(a ; m, n)=V^{\varepsilon}(a ; m, n)\left(1+K_{\varepsilon}^{-1} u^{\varepsilon}(a ; m, n)\right) .
$$

Of course we need to show that all other components in $\left(\mathcal{A}_{0}+\mathcal{A}_{c}\right) G_{\varepsilon}$, and $\mathcal{A}_{f} G_{\varepsilon}$ are small if $\varepsilon$ and $|z|$ are small. This can be achieved by rather straightforward reasoning if we require

$$
K_{\varepsilon}^{1 / 2}|z||\log | z|| \rightarrow 0, \quad K_{\varepsilon}^{-1 / 2}|\log | z|| \rightarrow 0 .
$$

(In higher dimension, the second condition is replaced with $K_{\varepsilon}^{-1 / 2}|z|^{2-d} \rightarrow 0$, which is inconsistent with the first condition if $d \geq 3$.) These two conditions are satisfied if $|z|=|\log \varepsilon|^{-\theta}$, for some $\theta>1 / 2$. At this stage, we simply use the smallness of $\hat{u}^{\varepsilon}$ for $z$ satisfying $\varepsilon<<|z|<<1$. In other words, we do not take advantage of the fact that $J$ is of 0 average and do not apply any central limit-type arguments. (For higher dimensions, this line of reasoning is not applicable and we really need to establish a central limit-type theorem to show that the error term in (4.8) is small.) Of course we may try to square the error term and take advantage of the fact that $J$ is of 0 average and that particles are independent at equilibrium. This turns out to be rather technical and challenging and will be dealt with in a future publication.

So far we have learned that the expression $Y_{\varepsilon}^{3}$ can be replaced with the right hand-side of (4.8). Once this is achieved, we take a smooth function $\zeta$ of compact support, set $\zeta^{\delta}(a)=\delta^{-d} \zeta\left(\frac{a}{\delta}\right)$, and define

$$
f_{n}^{\delta}(x, t)=K_{\varepsilon}^{-1} \sum_{i} \zeta^{\delta}\left(x_{i}(t)-x\right) \mathbb{1}\left(m_{i}(t)=n\right) .
$$

We think of this as an approximation of the density of particles of cluster size $n$. We choose $\delta=\delta(\varepsilon)=|\log \varepsilon|^{-\theta}$ with $\theta>1 / 2$. The outcome $\tilde{f}_{n}^{\varepsilon}(x, t)=f_{n}^{\delta(\varepsilon)}(x, t)$ converges weakly to $\lambda_{n}$ as $\varepsilon \rightarrow 0$. So far we have not carried out any CLT. We know that if $\left|z_{2}-z_{1}\right| \leq \delta(\varepsilon)$, then

$$
\int_{0}^{t} Y_{\varepsilon}^{3}(s) d s=\int_{0}^{t} B_{\varepsilon}^{z_{2}-z_{1}}(\omega(s)) d s+\int_{0}^{t} C_{\varepsilon}(\omega(s)) d s+D_{\varepsilon}(t)+\operatorname{Error}^{1}(\varepsilon)
$$

with $\lim _{\varepsilon \rightarrow 0} \operatorname{Error}^{1}(\varepsilon)=0$ and $D_{\varepsilon}(t)$ a martingale. We multiply both sides of (4.15) by $\zeta^{\delta}\left(z_{1}\right) \zeta^{\delta}\left(z_{2}\right)$ and integrate with respect to $z_{1}$ and $z_{2}$. After a change of variables $z_{1} \mapsto z_{1}-x_{i}, z_{2} \mapsto z_{2}-x_{j}$, we obtain

$$
\begin{aligned}
& \frac{1}{2} \int_{0}^{t} \iint K_{\varepsilon}^{-3 / 2} \sum_{i, j} \alpha\left(m_{i}, m_{j}\right) W^{\varepsilon}\left(z_{1}-z_{2} ; m_{i}, m_{j}\right) \tilde{J}\left(x_{i}, m_{i}, x_{j}, m_{j}\right) \\
& \quad \zeta^{\delta(\varepsilon)}\left(x_{i}-z_{1}\right) \zeta^{\delta(\varepsilon)}\left(x_{j}-z_{2}\right) d z_{1} d z_{2} d s
\end{aligned}
$$


for the first term of the right-hand side of (4.15). Since $J$ is smooth and $\zeta$ is of compact support, we may replace $\tilde{J}\left(x_{i}, m_{i}, x_{j}, m_{j}\right)$ with $\tilde{J}\left(z_{1}, m_{i}, z_{2}, m_{j}\right)$ for an error of order $O(\delta(\varepsilon))$. We then carry out the summation over $i$ and $j$ to obtain

$$
\begin{gathered}
\frac{1}{2} \sum_{m, n} \alpha(m, n) \int_{0}^{t} \iint K_{\varepsilon}^{1 / 2} f_{n}^{\delta(\varepsilon)}\left(z_{1}, s\right) f_{m}^{\delta(\varepsilon)}\left(z_{2}, s\right) \\
W^{\varepsilon}\left(z_{1}-z_{2} ; m, n\right) \tilde{J}\left(z_{1}, m, z_{2}, n\right) d z_{1} d z_{2} d s .
\end{gathered}
$$

Since $J$ is of zero average, the integrand

$$
\Lambda:=K_{\varepsilon}^{1 / 2}\left(f_{n}^{\delta(\varepsilon)}\left(z_{1}, s\right) f_{m}^{\delta(\varepsilon)}\left(z_{2}, s\right)-\lambda_{n} \lambda_{m}\right) W^{\varepsilon}\left(z_{1}-z_{2} ; m, n\right) \tilde{J}\left(z_{1}, m, z_{2}, n\right),
$$

can be written as

$$
\Lambda=\Lambda_{1}+\Lambda_{2}+\Lambda_{3}
$$

where

$$
\begin{aligned}
& \Lambda_{1}=K_{\varepsilon}^{1 / 2}\left(f_{n}^{\delta(\varepsilon)}\left(z_{1}, s\right)-\lambda_{n}\right) \lambda_{m} W^{\varepsilon}\left(z_{1}-z_{2} ; m, n\right) \tilde{J}\left(z_{1}, m, z_{2}, n\right), \\
& \Lambda_{2}=K_{\varepsilon}^{1 / 2}\left(f_{m}^{\delta(\varepsilon)}\left(z_{1}, s\right)-\lambda_{m}\right) \lambda_{n} W^{\varepsilon}\left(z_{1}-z_{2} ; m, n\right) \tilde{J}\left(z_{1}, m, z_{2}, n\right), \\
& \Lambda_{3}=K_{\varepsilon}^{1 / 2}\left[\left(f_{n}^{\delta(\varepsilon)}\left(z_{1}, s\right)-\lambda_{n}\right)\left(f_{m}^{\delta(\varepsilon)}\left(z_{2}, s\right)-\lambda_{m}\right)\right] W^{\varepsilon}\left(z_{1}-z_{2} ; m, n\right) \tilde{J}\left(z_{1}, m, z_{2}, n\right) .
\end{aligned}
$$

To achieve our goals, we wish to show that $\Lambda_{3}$ is small in average. Formally, if $\Lambda_{1}$ is a bounded quantity, then $\Lambda_{3}$ is smaller than $\Lambda_{1}$ because of the additional small term $f_{m}^{\delta(\varepsilon)}-\lambda_{m}$. This turns out to be wrong; the term $f_{m}^{\delta}-\lambda_{m}$ is small only in a weak sense and its product with $f_{n}^{\delta}-\lambda_{n}$ is no longer small. This is not surprising at all because $\delta=\delta(\varepsilon)$ is not sufficiently large enough for a central limit theorem to take place. Indeed the support of $\zeta^{\delta}$ is a set of volume $O\left(\delta^{d}\right)$ and as a result, the particle density $f_{n}^{\delta}$ involves $O\left(K_{\varepsilon} \delta^{d}\right)$ many particles in average. For a CLT taking place, we need a density which deals with a large number of particles. In other words, we expect $\Lambda_{3}$ to be small only when $K_{\varepsilon} \delta^{d} \rightarrow \infty$ as $\varepsilon \rightarrow \infty$. This would not be the case if $\delta=|\log \varepsilon|^{-\theta}$ for a $\theta>1 / 2$.

In order to figure out a successful way of going beyond $|\log \varepsilon|^{-\theta}$ and reach a density $f^{\delta}$ with $\delta$ satisfying $K_{\varepsilon} \delta^{d} \rightarrow \infty$, we need to review what has been achieved so far and what to learn from it.

Basically our goal is a central limit theorem (CLT) for the particle density (4.1) and for this we need to perform some type of CLT for the time average of (4.6). Note that $Y_{\varepsilon}^{3}$ is in some sense singular because the function $V^{\varepsilon}$ is a delta-type expression. That is, in a region of volume $O\left(\varepsilon^{d}\right), V^{\varepsilon}$ is of order $O\left(\varepsilon^{-d}\right)$. In fact if we calculate $\mathbb{E}_{\varepsilon}^{e q} Y_{\varepsilon}^{3}(\omega)^{2}$, we get an expression that blows up as $\varepsilon \rightarrow 0$. All this ultimately stems from the fact that the coagulation occurs when particles are microscopically close. We wish to replace $V^{\varepsilon}$ with a smoother kernel and this is exactly what purpose (4.8) serves. We try to replace $x_{i}-x_{j}$, the argument of $V^{\varepsilon}$, with $x_{i}-x_{j}+z$. That is, we try to figure out the coagulation rate when particles $x_{i}$ and $x_{j}$ are not microscopically close but only macroscopically close, i.e., $x_{i}-x_{j}=z+O(\varepsilon)$ with $|z| \rightarrow 0$ after sending $\varepsilon \rightarrow 0$. (For example $|z|$ could be as "large" as $|\log \varepsilon|^{-\theta}$.) However there is a price to pay for such a replacement; we need to replace $V^{\varepsilon}$ with $W^{\varepsilon}$ and modify the fragmentation term (we are referring to the term $C_{\varepsilon}$ ), and even the noise is modified (the term $D_{\varepsilon}$ ). To carry this out, we encountered various additional terms which are presumably small. We have a relatively easy ride, if $|z|<<|\log \varepsilon|^{-1 / 2}$. Even though we have not reached our ultimate goal $|z|>>|\log \varepsilon|^{-1 / 2}$, we have already achieved three important tasks: 
(i) The correctors $C_{\varepsilon}$ and $D_{\varepsilon}$ would modify the fragmentation and martingle terms as required in the proof of the main result Theorem 3.1.

(ii) The term $W^{\varepsilon}$ would allow us to replace $\alpha$ with $\hat{\alpha}$ because $\lim \int W^{\varepsilon}=\eta$ as $\varepsilon \rightarrow 0$.

(iii) We have been able to replace the singular term $V^{\varepsilon}(a ; m, n)$ with a less singular term $\bar{W}^{\varepsilon}(a ; m, n)=\int W^{\varepsilon}(a+z ; m, n) \zeta^{\delta(\varepsilon)}(z) d z$, where $\delta(\varepsilon)=|\log \varepsilon|^{-\theta}$ for some $\theta>1 / 2$.

We are now in a position to explain the central role of Eq. (4.11). Because of the time average in $\int_{0}^{t} Y_{\varepsilon}^{3}(s) d s$, we are dealing with an expression which is almost as smooth as $\mathcal{A}^{-1} Y_{\varepsilon}^{3}$. Of course $\mathcal{A}^{-1}$ is too complicated to use. The message behind Eq. (4.11) and its use is that we only need to consider 2-particles dynamics. Namely, the fact that $x_{i}-x_{j}$ is a diffusion with generator $\left(d\left(m_{i}\right)+d\left(m_{j}\right)\right) \Delta$, and that once a coagulation occurs with rate $\alpha\left(m_{i}, m_{j}\right)$ between the $i^{\text {th }}$ and $j^{\text {th }}$ particles, $\left(x_{i}, x_{j}\right)$ as a pair no longer exists and hence the dynamics of $x_{i}-x_{j}$ has an infinitesimal generator of a killed Brownian motion:

$$
\Gamma^{\varepsilon}=(d(m)+d(n)) \Delta-\alpha(m, n) V_{\varepsilon}(\cdot ; m, n),
$$

with $m=m_{i}$ and $n=m_{j}$. Now the function $u^{\varepsilon}=\Gamma_{\varepsilon}^{-1} V^{\varepsilon}$ is smoother than $V^{\varepsilon}$ and this allows us to perturb its argument by a small vector $z$ and obtain (4.8). By (iii), we are now dealing $\bar{W}^{\varepsilon}$ in place of $V^{\varepsilon}$. We note that $\bar{W}^{\varepsilon}=O\left(\delta(\varepsilon)^{-d}\right)$, and has a support of diameter $O(\delta(\varepsilon))$. To replace $\bar{W}^{\varepsilon}$ with $\tilde{W}^{\varepsilon}(a ; m, n)=\int W^{\varepsilon}(a+z ; m, n) \zeta^{\delta^{\prime}(\varepsilon)}(z) d z$, for some $\delta^{\prime}(\varepsilon)>>|\log \varepsilon|^{-1 / 2}$, we almost repeat the formula (4.12) where $V^{\varepsilon}$ is replaced with $\bar{W}^{\varepsilon}$, and $u^{\varepsilon}$ is replaced with $v^{\varepsilon}$ which now solves

$$
(d(m)+d(n)) \Delta v^{\varepsilon}(x ; m, n)=\alpha(m, n) \bar{W}^{\varepsilon}(x ; m, n) .
$$

This time we can show that various terms that appear in $\mathcal{A} G_{\varepsilon}$ are small provided that $|z| \leq \delta^{\prime}(\varepsilon)$ for $\delta^{\prime}$, that is, now can be chosen as large as $|\log \log \varepsilon|^{-\theta^{\prime}}$ for any $\theta^{\prime}>1 / 2$. For this step of the proof we show that all the error terms have small second moments, in other words, a CLT is taking place and the errors have small variances. (see Sect.7).

As a consequence of the main result of Sect.7, we have the decomposition (4.15) where $\delta(\varepsilon)$ is replaced with $\delta^{\prime}(\varepsilon)$. We can now rigorously show that $\Lambda_{3}$ is small by ignoring the time integration and showing that the integrand has a small second moment with respect to the equilibrium measure. As for $\Lambda_{1}$, we first carry out $d z_{2}$ integration and use the fact that $\lim \int W^{\varepsilon}(a ; m, n) d a=\eta(m, n)$, as $\varepsilon \rightarrow 0$. (This was proved as Theorem 3.2 in [HR2].) After some straightforward manipulations,

$$
\int_{0}^{t} \iint \Lambda_{1} d z_{1} d z_{2} d s=\left[\int_{0}^{t} \xi_{n}(t, J) d s\right] \eta(m, n) \lambda_{m}+\operatorname{Error}^{2}(\varepsilon) .
$$

As for $\Lambda_{2}$, we first replace $J\left(z_{1}\right)$ with $J\left(z_{2}\right)$ for a small error because $\left|z_{1}-z_{2}\right|=O(\varepsilon)$. We then integrate with respect to $z_{1}$ and repeat our reasoning for $\Lambda_{1}$ to obtain

$$
\int_{0}^{t} \iint \Lambda_{2} d z_{1} d z_{2} d s=\left[\int_{0}^{t} \xi_{m}(s, J) d s\right] \eta(m, n) \lambda_{n}+\operatorname{Error}^{3}(\varepsilon) .
$$

In summary

$$
\begin{aligned}
\int_{0}^{t} Y_{\varepsilon}^{3}(s) d s= & \frac{1}{2} \sum_{m, n} \hat{\alpha}(m, n) \int_{0}^{t}\left(\xi_{m}(s, J) \lambda_{n}+\xi_{n}(s, J) \lambda_{m}\right) d s \\
& +D_{\varepsilon}(t)+\operatorname{Error}(\varepsilon)
\end{aligned}
$$

for an error $\operatorname{Error}(\varepsilon)$ that goes to 0 on $\varepsilon \rightarrow 0$. 


\section{Regularity of the Coagulation Term, Part I}

As we mentioned in Sect.4, the main ingredient for the proof of Theorem 3.1 is the statement (4.18). In this section this statement is partially established and the full proof of (4.18) will be achieved in Sect. 7.

We now prepare for the main result of this section, which will appear as Theorem 5.1 at the end of the section. The proof of Theorem 5.1 will be given in Sect. 6. Note that the function $J$ in (4.1) is of compact support and satisfies $\int J(x, n) d x=0$, for every $n$. In fact we only need to consider $J(x, n)=\bar{J}(x) \mathbb{1}(n=\bar{m})$ with $\int \bar{J}(x) d x=0$. Evidently for such a function $J$, we have $\int J(x, n) d x=0$ for every $n$. Note that $\tilde{J}$ of (4.7) is not of compact support. However, for some positive $l$, we have that $\tilde{J}(x, m, y, n)=0$, if either $m, n \geq l$ or $\left|x_{i}\right|,\left|x_{j}\right| \geq l$. Because of the $V_{\varepsilon}$ term in the definition of $Y_{\varepsilon}^{3}$, we may replace $\tilde{J}$ with

$$
\hat{J}\left(x_{i}, m_{i}, x_{j}, m_{j}\right)=\tilde{J}\left(x_{i}, m_{i}, x_{j}, m_{j}\right) K\left(x_{i}-x_{j}\right),
$$

for a smooth symmetric function $K(a)$ of compact support which is 1 whenever $|a| \leq 1$. The advantage of $\hat{J}$ to $\tilde{J}$ is that the former is of compact support in the spatial variables. Note however, the term $V_{\varepsilon}$ only implies that $\left|x_{i}-x_{j}\right| \leq c_{0} \varepsilon r\left(m_{i}, m_{j}\right)$ for a constant $c_{0}$. Hence such a replacement is valid only if $c_{0} \varepsilon r\left(m_{i}, m_{j}\right) \leq 1$. This causes an error that can be readily handled with the aid of our hypothesis (3.9). (See the first step of the proof of Theorem 8.1 in Sect. 8.)

Recall that $u^{\varepsilon}(x ; m, n)$ solves

$$
(d(m)+d(n)) \Delta u^{\varepsilon}(x ; m, n)=\alpha(m, n)\left[V_{\varepsilon}(x ; m, n) u^{\varepsilon}(x ; m, n)+V^{\varepsilon}(x ; m, n)\right],
$$

where $V^{\varepsilon}(x ; m, n)=\varepsilon^{-2} V(x / \varepsilon ; m, n)$, and $V_{\varepsilon}(x ; m, n)=K_{\varepsilon}^{-1} \varepsilon^{-2} V(x / \varepsilon ; m, n)$. Given such a function $u^{\varepsilon}$, we define

$$
G(\omega ; z)=G(\omega)=K_{\varepsilon}^{-3 / 2} \sum_{i, j} \hat{u}^{\varepsilon}\left(x_{i}-x_{j} ; m_{i}, m_{j}\right) \hat{J}\left(x_{i}, m_{i}, x_{j}, m_{j}\right)
$$

where $\hat{u}^{\varepsilon}(a ; m, n)=u^{\varepsilon}(a+z ; m, n)-u^{\varepsilon}(a ; m, n)$. We have

$$
G(\omega(t))=G(\omega(0))+\int_{0}^{t} \mathcal{A} G(\omega(s)) d s+M_{t}
$$

where $M_{t}$ is a martingale. We write

$$
\mathcal{A} G=\mathcal{A}_{0} G+\mathcal{A}_{c} G+\mathcal{A}_{f} G=: H_{1}+H_{2}+H_{3}
$$

We now study various terms which appeared on the right-hand side. We write $\hat{J}_{x}$ and $\hat{J}_{y}$ for the derivatives of $\hat{J}$ with respect to its first and second spatial arguments. We then write

$$
H_{1}=H_{11}+H_{12}+H_{13},
$$


with

$$
\begin{aligned}
H_{11}(\omega)= & K_{\varepsilon}^{-3 / 2} \sum_{i, j} \hat{u}^{\varepsilon}\left(x_{i}-x_{j} ; m_{i}, m_{j}\right)\left[\left(d\left(m_{i}\right) \Delta_{x_{i}}+d\left(m_{j}\right) \Delta_{x_{j}}\right) \hat{J}\left(x_{i}, m_{i}, x_{j}, m_{j}\right)\right], \\
H_{12}(\omega)= & K_{\varepsilon}^{-3 / 2} \sum_{i, j} d\left(m_{i}\right) \hat{u}_{x}^{\varepsilon}\left(x_{i}-x_{j} ; m_{i}, m_{j}\right) \cdot \hat{J}_{x}\left(x_{i}, m_{i}, x_{j}, m_{j}\right) \\
& -K_{\varepsilon}^{-3 / 2} \sum_{i, j} d\left(m_{j}\right) \hat{u}_{x}^{\varepsilon}\left(x_{i}-x_{j} ; m_{i}, m_{j}\right) \cdot \hat{J}_{y}\left(x_{i}, m_{i}, x_{j}, m_{j}\right) \\
=: & H_{121}(\omega)-H_{122}(\omega), \\
H_{13}(\omega)= & K_{\varepsilon}^{-3 / 2} \sum_{i, j}\left(d\left(m_{i}\right)+d\left(m_{j}\right)\right) \Delta \hat{u}^{\varepsilon}\left(x_{i}-x_{j} ; m_{i}, m_{j}\right) \hat{J}\left(x_{i}, m_{i}, x_{j}, m_{j}\right) \\
=: & H_{13}^{z}(\omega)-H_{131}^{0}(\omega)-H_{132}^{0}(\omega),
\end{aligned}
$$

where

$$
H_{13}^{z}(\omega)=K_{\varepsilon}^{-3 / 2} \sum_{i, j} \alpha\left(m_{i}, m_{j}\right) W^{\varepsilon}\left(x_{i}-x_{j}+z ; m_{i}, m_{j}\right) \hat{J}\left(x_{i}, m_{i}, x_{j}, m_{j}\right)
$$

with $W^{\varepsilon}(a ; m, n)=u^{\varepsilon}(a ; m, n) V_{\varepsilon}(a ; m, n)+V^{\varepsilon}(a ; m, n)$, and

$$
\begin{aligned}
H_{131}^{0}(\omega)= & K_{\varepsilon}^{-3 / 2} \sum_{i, j} \alpha\left(m_{i}, m_{j}\right) u^{\varepsilon}\left(x_{i}-x_{j} ; m_{i}, m_{j}\right) \\
& V_{\varepsilon}\left(x_{i}-x_{j} ; m_{i}, m_{j}\right) \hat{J}\left(x_{i}, m_{i}, x_{j}, m_{j}\right), \\
H_{132}^{0}(\omega)= & K_{\varepsilon}^{-3 / 2} \sum_{i, j} \alpha\left(m_{i}, m_{j}\right) V^{\varepsilon}\left(x_{i}-x_{j} ; m_{i}, m_{j}\right) \hat{J}\left(x_{i}, m_{i}, x_{j}, m_{j}\right) .
\end{aligned}
$$

We also write

$$
H_{2}=H_{21}+H_{22}, \quad H_{21}=H_{21}^{z}-H_{21}^{0},
$$

with $H_{21}^{z}(\omega)$ given by

$$
\begin{gathered}
-\frac{1}{2} K_{\varepsilon}^{-3 / 2} \sum_{i, j} \alpha\left(m_{i}, m_{j}\right) V_{\varepsilon}\left(x_{i}-x_{j} ; m_{i}, m_{j}\right) \\
{\left[u^{\varepsilon}\left(x_{i}-x_{j}+z ; m_{i}, m_{j}\right) \hat{J}\left(x_{i}, m_{i}, x_{j}, m_{j}\right)\right.} \\
\left.+u^{\varepsilon}\left(x_{j}-x_{i}+z ; m_{i}, m_{j}\right) \hat{J}\left(x_{j}, m_{j}, x_{i}, m_{i}\right)\right] \\
=-K_{\varepsilon}^{-3 / 2} \sum_{i, j} \alpha\left(m_{i}, m_{j}\right) V_{\varepsilon}\left(x_{i}-x_{j} ; m_{i}, m_{j}\right) \\
u^{\varepsilon}\left(x_{i}-x_{j}+z ; m_{i}, m_{j}\right) \hat{J}\left(x_{i}, m_{i}, x_{j}, m_{j}\right) .
\end{gathered}
$$


Moreover,

$$
\begin{aligned}
H_{22}(\omega)= & \frac{1}{2} \sum_{i, j} \alpha\left(m_{i}, m_{j}\right) V_{\varepsilon}\left(x_{i}-x_{j} ; m_{i}, m_{j}\right) K_{\varepsilon}^{-3 / 2} \\
& \sum_{k}\left\{\frac { m _ { i } } { m _ { i } + m _ { j } } \left[\hat{u}^{\varepsilon}\left(x_{i}-x_{k} ; m_{i}+m_{j}, m_{k}\right) \hat{J}\left(x_{i}, m_{i}+m_{j}, x_{k}, m_{k}\right)\right.\right. \\
& \left.+\hat{u}^{\varepsilon}\left(x_{k}-x_{i}, m_{k}, m_{i}+m_{j}\right) \hat{J}\left(x_{k}, m_{k}, x_{i}, m_{i}+m_{j}\right)\right] \\
& +\frac{m_{i}}{m_{i}+m_{j}}\left[\hat{u}^{\varepsilon}\left(x_{j}-x_{k} ; m_{i}+m_{j}, m_{k}\right) \hat{J}\left(x_{j}, m_{i}+m_{j}, x_{k}, m_{k}\right)\right. \\
& \left.+\hat{u}^{\varepsilon}\left(x_{k}-x_{j} ; m_{k}, m_{i}+m_{j}\right) \hat{J}\left(x_{k}, m_{k}, x_{j}, m_{i}+m_{j}\right)\right] \\
& -\left[\hat{u}^{\varepsilon}\left(x_{i}-x_{k} ; m_{i}, m_{n}\right) \hat{J}\left(x_{i}, m_{i}, x_{k}, m_{k}\right)\right. \\
& \left.+\hat{u}^{\varepsilon}\left(x_{k}-x_{i} ; m_{k}, m_{i}\right) \hat{J}\left(x_{k}, m_{k}, x_{i}, m_{i}\right)\right] \\
& -\left[\hat{u}^{\varepsilon}\left(x_{j}-x_{k} ; m_{j}, m_{k}\right) \hat{J}\left(x_{j}, m_{j}, x_{k}, m_{k}\right)\right. \\
& \left.\left.+\hat{u}^{\varepsilon}\left(x_{k}-x_{j} ; m_{k}, m_{j}\right) \hat{J}\left(x_{k}, m_{k}, x_{j}, m_{j}\right)\right]\right\}
\end{aligned}
$$

The expression $H_{22}$ arises from the changes in the function $G$ when a coagulation occurs due to the influence of the appearance and disappearance of particles on other particles that are not directly involved. The expression $H_{21}$ represents those terms in $G$ that are absent after a coagulation. Note that for our formula for $H_{12}$, we used the fact that $K$ is symmetric and since $V$ is symmetric, the function $u^{\varepsilon}$ is also symmetric.

As for the fragmentation part of dynamics, we have

$$
H_{3}=H_{31}+H_{32}+H_{33},
$$

where $H_{31}=H_{311}+H_{312}$, with

$$
\begin{aligned}
H_{311}(\omega)= & \frac{1}{2} \int K_{\varepsilon}^{-3 / 2} \sum_{i, j} \sum_{m=1}^{m_{i}-1} \beta\left(m, m_{i}-m\right) V^{\varepsilon}\left(x_{i}-y ; m, m_{i}-m\right) \\
& \quad\left[\hat{u}^{\varepsilon}\left(x_{i}-x_{j} ; m, m_{j}\right) \hat{J}\left(x_{i}, m, x_{j}, m_{j}\right)\right. \\
& \left.\quad-\hat{u}^{\varepsilon}\left(x_{i}-x_{j} ; m_{i}, m_{j}\right) \hat{J}\left(x_{i}, m_{i}, x_{j}, m_{j}\right)\right] d y, \\
H_{312}(\omega)= & \frac{1}{2} \int K_{\varepsilon}^{-3 / 2} \sum_{i, j} \sum_{m=1}^{m_{j}-1} \beta\left(m, m_{j}-m\right) V^{\varepsilon}\left(x_{j}-y ; m, m_{j}-m\right) \\
& {\left[\hat{u}^{\varepsilon}\left(x_{i}-x_{j} ; m_{i}, m\right) \hat{J}\left(x_{i}, m_{i}, x_{j}, m\right)\right.} \\
& \left.\quad-\hat{u}^{\varepsilon}\left(x_{i}-x_{j} ; m_{i}, m_{j}\right) \hat{J}\left(x_{i}, m_{i}, x_{j}, m_{j}\right)\right] d y .
\end{aligned}
$$


We carry out $d y$ integration and use symmetry to obtain that $H_{31}=2 H_{311}$, where

$$
\begin{aligned}
& H_{311}(\omega)=\frac{1}{2} \int K_{\varepsilon}^{-3 / 2} \sum_{i, j} \sum_{m=1}^{m_{i}-1} \beta\left(m, m_{i}-m\right) \\
& {\left[\hat{u}^{\varepsilon}\left(x_{i}-x_{j} ; m, m_{j}\right) \hat{J}\left(x_{i}, m, x_{j}, m_{j}\right)\right.} \\
& \left.-\hat{u}^{\varepsilon}\left(x_{i}-x_{j} ; m_{i}, m_{j}\right) \hat{J}\left(x_{i}, m_{i}, x_{j}, m_{j}\right)\right] .
\end{aligned}
$$

Also, $H_{32}=H_{321}+H_{322}$, with

$$
\begin{aligned}
H_{321}(\omega)= & \frac{1}{2} \int K_{\varepsilon}^{-3 / 2} \sum_{i, j} \sum_{m=1}^{m_{i}-1} \beta\left(m, m_{i}-m\right) V^{\varepsilon}\left(x_{i}-y ; m, m_{i}-m\right) \\
& \hat{u}^{\varepsilon}\left(y-x_{j} ; m, m_{j}\right) \hat{J}\left(y, m, x_{j}, m_{j}\right) d y, \\
H_{322}(\omega)= & \frac{1}{2} \int K_{\varepsilon}^{-3 / 2} \sum_{i, j} \sum_{m=1}^{m_{j}-1} \beta\left(m, m_{j}-m\right) V^{\varepsilon}\left(x_{j}-y ; m, m_{j}-m\right) \\
& \hat{u}^{\varepsilon}\left(x_{i}-y ; m_{i}, m\right) \hat{J}\left(x_{i}, m_{i}, y, m\right) d y,
\end{aligned}
$$

and $H_{33}=H_{33}^{z}-H_{33}^{0}$, with

$$
\begin{aligned}
H_{33}^{z}(\omega)= & \int K_{\varepsilon}^{-3 / 2} \sum_{i} \sum_{m=1}^{m_{i}-1} \beta\left(m, m_{i}-m\right) V^{\varepsilon}\left(x_{i}-y ; m, m_{i}-m\right) \\
& u^{\varepsilon}\left(x_{i}-y+z ; m, m_{i}-m\right) \hat{J}\left(x_{i}, m, y, m_{i}-m\right) d y .
\end{aligned}
$$

Note that $H_{131}^{0}+H_{21}^{0}=0$. We may rewrite (5.2) as

$$
\mathcal{A} G+\left[H_{132}^{0}-H_{13}^{z}+H_{33}^{0}\right]=\left(H_{11}+H_{12}\right)+H_{21}^{z}+\left(H_{22}+H_{31}+H_{32}\right)+H_{33}^{z} .
$$

We are now ready to state the main result of this section.

Theorem 5.1. Let $\hat{J}$ be as above and assume that $\sqrt{\varepsilon}<|z|<1$. Then

$$
\begin{aligned}
& \mathbb{E}_{\varepsilon}^{e q}\left|\int_{0}^{t} \mathcal{A} G(\omega(s)) d s+\int_{0}^{t}\left[H_{132}^{0}(\omega(s))-H_{13}^{z}(\omega(s))+H_{33}^{0}(\omega(s))\right] d s\right| \\
& \quad \leq C_{0} t\left[K_{\varepsilon}^{1 / 2}|z||\log | z||+K_{\varepsilon}^{-1 / 2}|\log | z||\right] .
\end{aligned}
$$

We establish Theorem 5.1 by examining various terms that appeared on the right-hand side of (5.3). Indeed we show

$$
\begin{gathered}
\mathbb{E}_{\varepsilon}^{e q}|G(\omega(t))||G(\omega(0))| \leq C_{0}^{\prime} K_{\varepsilon}^{1 / 2}|z|, \\
\mathbb{E}_{\varepsilon}^{e q}\left|H_{11}(\omega(s))\right| \leq C_{0}^{\prime} K_{\varepsilon}^{1 / 2}|z|, \\
\mathbb{E}_{\varepsilon}^{e q}\left|H_{12}(\omega(s))\right| \leq C_{0}^{\prime} K_{\varepsilon}^{1 / 2}|z||\log | z||,
\end{gathered}
$$




$$
\begin{gathered}
\mathbb{E}_{\varepsilon}^{e q}\left|H_{22}(\omega(s))\right| \leq C_{0}^{\prime} K_{\varepsilon}^{1 / 2}|z|, \\
\mathbb{E}_{\varepsilon}^{e q}\left|H_{31}(\omega(s))\right| \leq C_{0}^{\prime} K_{\varepsilon}^{1 / 2}|z|, \\
\mathbb{E}_{\varepsilon}^{e q}\left|H_{32}(\omega(s))\right| \leq C_{0}^{\prime} K_{\varepsilon}^{1 / 2}|z|, \\
\mathbb{E}_{\varepsilon}^{e q}\left|H_{21}^{z}(\omega(s))\right| \leq C_{0}^{\prime} K_{\varepsilon}^{-1 / 2}|\log | z||, \\
\mathbb{E}_{\varepsilon}^{e q}\left|H_{33}^{z}(\omega(s))\right| \leq C_{0}^{\prime} K_{\varepsilon}^{-1 / 2}|\log | z|| .
\end{gathered}
$$

Theorem 5.1 is an immediate consequence of (5.5-11). The bound (5.5) will be used for the proof of Theorem 3.1.

As we mentioned in Sect.4, our method of proof can be used to establish a law of large number (LLN) for the expression $\int_{0}^{t} K_{\varepsilon}^{1 / 2} Y_{\varepsilon}^{3}(s) d s$ with $Y_{\varepsilon}^{3}$ as in (4.4). This can be achieved as in [HR2] by using the regularity of the coagulation term and this time $z$ can be chosen to be any small vector. Moreover for $\tilde{J}$, we may choose any smooth function of compact support. Note that since we are at equilibrium, the proof of LLN is much easier than what we have in [HR2] because all the correlation bounds needed for the proof are trivially true. This would allow us to find the limit of $\int_{0}^{t} K_{\varepsilon}^{1 / 2} Y_{\varepsilon}^{3}(s) d s$ as $\varepsilon \rightarrow 0$. Since this limit is not random, the limit can be calculated by passing to the limit in $\mathbb{E}_{\varepsilon}^{e q} \int_{0}^{t} K_{\varepsilon}^{1 / 2} Y_{\varepsilon}^{3}(s) d s=t \mathbb{E}_{\varepsilon}^{e q} K_{\varepsilon}^{1 / 2} Y_{\varepsilon}^{3}(0)$. In summary,

Lemma 5.1. Let $K(x, m, y, n)$ by any smooth function of compact support. Then

$$
\lim _{\varepsilon \rightarrow 0} \mathbb{E}_{\varepsilon}^{e q}\left|\int_{0}^{t} Z^{\varepsilon}(\omega(s)) d s-t \bar{Z}\right|=0,
$$

where

$$
\begin{aligned}
Z^{\varepsilon}(\omega) & =K_{\varepsilon}^{-2} \sum_{i, j} \alpha\left(m_{i}, m_{j}\right) V^{\varepsilon}\left(x_{i}-x_{j} ; m_{i}, m_{j}\right) K\left(x_{i}, m_{i}, x_{j}, m_{j}\right), \\
\bar{Z} & =\sum_{m, n} \lambda_{m} \lambda_{n} \alpha(m, n) \int K(x, m, x, n) d x
\end{aligned}
$$

Lemma 5.1 will be needed in Sect. 8. In Sect. 8 we also need another LLN which can be established with a similar argument. This time our $Z^{\varepsilon}(\omega)$ is given by

$$
\begin{gathered}
K_{\varepsilon}^{-2} \sum_{i, j} d\left(m_{i}\right)\left(K_{\varepsilon}^{-1}\left|\nabla u^{\varepsilon}\left(x_{i}-x_{j} ; m_{i}, m_{j}\right)\right|^{2}\right) \\
\tilde{J}\left(x_{i}, m_{i}, x_{i}, m_{j}\right)^{2} \mathbb{1}\left(\left|x_{i}-x_{j}\right| \leq 1\right) .
\end{gathered}
$$

As we will see in Lemma 6.1 of Sect. 6, the function

$$
W^{\varepsilon}(a ; m, n)=K_{\varepsilon}^{-1}\left|\nabla u^{\varepsilon}(a ; m, n)\right|^{2} 11(|a| \leq 1),
$$

is almost as singular as $V^{\varepsilon}(a ; m, n)$ because $W^{\varepsilon}(a ; m, n)=O\left(\varepsilon^{-2} K_{\varepsilon}^{-1}\right)$ when $|a| \leq \varepsilon$. However $\int W^{\varepsilon} d a$ stays bounded as $\varepsilon \rightarrow 0$. We will calculate $\gamma=\lim _{\varepsilon \rightarrow 0} \int W^{\varepsilon} d \bar{a}$ in Sect. 8 (see the final step of the proof of (8.4).) We have, 
Lemma 5.2. Let $Z^{\varepsilon}$ be as in (5.14). Then (5.13) is true for

$$
\bar{Z}=\sum_{m, n} \lambda_{m} \lambda_{n} d(m) \gamma(m, n) \int \tilde{J}(x, m, x, n)^{2} d x
$$

This lemma can be proved in a similar way. This time we start with a function $w^{\varepsilon}(x ; m, n)$ that now solves

$(d(m)+d(n)) \Delta w^{\varepsilon}(x ; m, n)=\alpha(m, n) V_{\varepsilon}(x ; m, n) w^{\varepsilon}(x ; m, n)+d(m) W^{\varepsilon}(x ; m, n)$,

and define

$$
G(\omega)=K_{\varepsilon}^{-2} \sum_{i, j} \hat{w}^{\varepsilon}\left(x_{i}-x_{j} ; m_{i}, m_{j}\right) \tilde{J}\left(x_{i}, m_{i}, x_{i}, m_{j}\right)^{2},
$$

where $\hat{w}^{\varepsilon}(a ; m, n)=w^{\varepsilon}(a+z ; m, n)-w^{\varepsilon}(a ; m, n)$. Again, using the same method of proof as [HR2] we can show that the limit in (5.13) exists and then by taking the expectation of $Z^{\varepsilon}$, we identify the limit.

\section{Proof of Theorem 5.1}

In this section, we establish (5.5) - (5.12). As a preliminary step, we state a lemma about the regularity of the function $u^{\varepsilon}$. Recall that $u^{\varepsilon}$ satisfies (4.11) or equivalently

$$
(d(m)+d(n)) \Delta_{x} u^{\varepsilon}(x ; m, n)=\alpha(m, n) V^{\varepsilon}(x ; m, n)\left[|\log \varepsilon|^{-1} u^{\varepsilon}(x ; m, n)+1\right],
$$

In fact $\log \varepsilon \leq u^{\varepsilon}$ and $u^{\varepsilon}$ is given by

$$
\frac{1}{2 \pi} \alpha^{\prime}(m, n) \int \log |x-y| V^{\varepsilon}(y ; m, n)\left[|\log \varepsilon|^{-1} u^{\varepsilon}(y ; m, n)+1\right] d y,
$$

where $\alpha^{\prime}(m, n)=\alpha(m, n) /(d(m)+d(n))$.

To ease the notation, we do not display the dependence of $\alpha^{\prime}(m, n)$ and $r(m, n)$ on $m$ and $n$.

Lemma 6.1. There exist positive constants $C_{1}$ and $C_{2}$ such that for all $x$,

$$
\begin{gathered}
\left|u^{\varepsilon}(x ; m, n)\right| \leq C_{1} \alpha^{\prime} \min \left\{1+\left|\log \frac{|x|}{r}\right|,|\log \varepsilon|\right\}, \\
\left|\nabla u^{\varepsilon}(x ; m, n)\right| \leq C_{1} \alpha^{\prime} \min \left\{|x|^{-1},(r \varepsilon)^{-1}\right\}
\end{gathered}
$$

and for $|x| \geq 2|z|+C_{2} r \varepsilon$,

$$
\left|\nabla u^{\varepsilon}(x+z ; m, n)-\nabla u^{\varepsilon}(x ; m, n)\right| \leq C_{1} \alpha^{\prime}|x|^{-2}|z| .
$$


Also,

$$
\begin{gathered}
\int_{|a| \leq l}\left|\nabla u^{\varepsilon}(a ; m, n)\right| d a \leq C_{1} \alpha^{\prime} l \\
\int_{|a| \leq l}\left|\hat{u}^{\varepsilon}(a ; m, n)\right| d a \leq C_{1} \alpha^{\prime}(l+|z|)|z| \\
\int_{|a| \leq l}\left|\nabla \hat{u}^{\varepsilon}(a ; m, n)\right| d a \leq C_{1} \alpha^{\prime}\left\{|z|\left[|\log (|z|+r \varepsilon)|+1+\log ^{+} l\right]+r \varepsilon\right\} \\
\int_{|a| \leq l} u^{\varepsilon}(a ; m, n)^{2} d a \leq C_{1} \alpha^{\prime 2}\left[r^{2} \varepsilon^{2}|\log \varepsilon|^{2}+l^{2}\left(\log ^{+} \frac{l}{r}\right)^{2}+1\right] \\
\int_{|a| \leq l}\left|\nabla u^{\varepsilon}(a ; m, n)\right|^{2} d a \leq C_{1} \alpha^{\prime 2}\left[1+\log ^{+} \frac{l}{r \varepsilon}+r^{2}\right] .
\end{gathered}
$$

Proof. The proofs of (6.1), (6.2) and (6.3) are omitted and can be found in Sect. 2.2 of [HR2]. Note however that in [HR2] we are assuming that $\chi=0$ and that we were dealing with $V^{\varepsilon}(x)=\varepsilon^{-2} V(x / \varepsilon)$ instead of $(\varepsilon r)^{-2} V(x /(r \varepsilon))$. Since we have $u^{\varepsilon}(x ; m, n)=$ $v^{\varepsilon}(x / r)$ for $r=r(m, n)$ and $v^{\varepsilon}$ solving

$$
(d(n)+d(m)) \Delta v^{\varepsilon}(x)=\alpha(m, n) V^{\varepsilon}(x)\left[|\log \varepsilon|^{-1} v^{\varepsilon}(x)+1\right],
$$

we can readily use the results of [HR2] to obtain (6.1), (6.2) and (6.3).

As for (6.4), we apply (6.2) to assert

$$
\int_{|a| \leq l}\left|\nabla u^{\varepsilon}(a ; m, n)\right| d a \leq c_{1} \alpha^{\prime} \int_{|a| \leq l} \min \left\{|a|^{-1},(r \varepsilon)^{-1}\right\} d a \leq c_{2} \alpha^{\prime} l .
$$

As for (6.5), we simply write,

$$
\begin{aligned}
\int_{|a| \leq l}\left|\hat{u}^{\varepsilon}(a ; m, n)\right| d a & =\int_{|a| \leq l}\left|\int_{0}^{1} \nabla u^{\varepsilon}(a+t z ; m, n) \cdot z d t\right| d a \\
& \leq|z| \int_{|a| \leq l+|z|}\left|\nabla u^{\varepsilon}(a ; m, n)\right| d a,
\end{aligned}
$$

and apply (6.4).

As for (6.6), we use (6.3) and (6.4) to write

$$
\begin{aligned}
\int\left|\nabla \hat{u}^{\varepsilon}(a ; m, n)\right| d a \leq & \int_{|a| \leq 2|z|+C_{2} r \varepsilon}\left|\nabla \hat{u}^{\varepsilon}(a ; m, n)\right| d a \\
& +C_{1} \int_{2|z|+C_{2} r \varepsilon \leq|a| \leq l} \alpha^{\prime}|a|^{-2}|z| d a \\
\leq & c_{2} \alpha^{\prime}[(|z|+r \varepsilon)+|z||\log (|z|+r \varepsilon)|+|z||\log l|] .
\end{aligned}
$$

For the proof of (6.7), let us write $A(l ; m, n)$ for the left-hand side of (6.7). We use (6.1) to assert that if $l \leq \varepsilon r$, then

$$
A(l ; m, n) \leq c_{2} l^{2} \alpha^{\prime 2}|\log \varepsilon|^{2} \leq c_{2} \alpha^{\prime 2} r^{2} \varepsilon^{2}|\log \varepsilon|^{2},
$$


and if $\varepsilon r<l$, then $A(l ; m, n)$ is bounded above by

$$
\begin{aligned}
& c_{3} \alpha^{2}\left[r^{2} \varepsilon^{2}|\log \varepsilon|^{2}+\int \mathbb{1}(|a| \in(\varepsilon r, l))\left|\log \frac{|a|}{r}\right| d a\right] \\
& \leq c_{4} \alpha^{\prime 2}\left[r^{2} \varepsilon^{2}|\log \varepsilon|^{2}+l^{2}\left|\log \frac{l}{r}\right|^{2}+1\right],
\end{aligned}
$$

completing the proof of (6.7). In the same fashion, we can readily establish (6.8).

Proof of (5.5), (5.6) and (5.7). We omit the proof of (5.6) because its proof is very similar to the proof of (5.5).

Evidently

$$
\begin{aligned}
\mathbb{E}_{\varepsilon}^{e q}|G(\omega(0))| & \leq c_{1} K_{\varepsilon}^{1 / 2} \int_{|a| \leq 1} \sum_{m, n} \lambda_{m} \lambda_{n}\left|\hat{u}^{\varepsilon}(a ; m, n)\right| d a \\
& \leq c_{2} K_{\varepsilon}^{1 / 2}|z| \sum_{m, n} \alpha^{\prime}(m, n) \lambda_{m} \lambda_{n} \leq c_{3} K_{\varepsilon}^{1 / 2}|z|,
\end{aligned}
$$

where we used (6.5) and (3.10) for the the second and third inequalities respectively. This proves (5.5).

We now turn to the proof of (5.7). We certainly have

$$
\begin{aligned}
\mathbb{E}_{\varepsilon}^{e q}\left|H_{12}(\omega(0))\right| & \leq c_{1} K_{\varepsilon}^{1 / 2} \int_{|a| \leq 1} \sum_{m, n} \lambda_{m} \lambda_{n}\left|\nabla \hat{u}^{\varepsilon}(a ; m, n)\right| d a \\
& \leq c_{2} K_{\varepsilon}^{1 / 2} \sum_{m, n} \alpha^{\prime}(m, n)(r(m, n) \varepsilon+|z| \log (|z|+r(m, n) \varepsilon)) \lambda_{m} \lambda_{n} \\
& \leq c_{3} K_{\varepsilon}^{1 / 2}|z||\log | z||,
\end{aligned}
$$

by (6.6) of Lemmas 6.2. We now use (3.10) to deduce (5.7).

Proof of (5.8). Evidently the expression $\mathbb{E}_{\varepsilon}^{e q}\left|H_{22}(\omega(0))\right|$ is bounded by

$$
\begin{aligned}
& c_{1} K_{\varepsilon}^{1 / 2} \iint_{|a| \leq 1} \sum_{m, n, p} \alpha(m, n) V^{\varepsilon}(b ; m, n)\left(\left|\hat{u}^{\varepsilon}(a ; m, p)\right|\right. \\
& \left.\quad+\left|\hat{u}^{\varepsilon}(a ; m+n, p)\right|\right) \lambda_{m} \lambda_{n} \lambda_{p} d a d b \\
& \leq c_{2} K_{\varepsilon}^{1 / 2}|z| \sum_{m, n, p} \alpha(m, n)\left(\alpha^{\prime}(n, p)\right. \\
& \left.\quad+\alpha^{\prime}(m+n, p)\right) \lambda_{m} \lambda_{n} \lambda_{p} \leq c_{3} K_{\varepsilon}^{1 / 2}|z|,
\end{aligned}
$$

where we used (6.5) and (3.10) for the second and third inequalities respectively. This proves (5.8).

Proof of (5.9) and (5.10). We start with the proof (5.9). We have $H_{311}=H_{3111}-H_{3112}$, where

$$
H_{3112}(\omega)=\frac{1}{2} \int K_{\varepsilon}^{-3 / 2} \sum_{i, j} \sum_{m=1}^{m_{i}-1} \beta^{\prime}\left(m_{i}\right) \hat{u}^{\varepsilon}\left(x_{i}-x_{j} ; m_{i}, m_{j}\right) \hat{J}\left(x_{i}, m_{i}, x_{j}, m_{j}\right),
$$


with

$$
\beta^{\prime}\left(m_{i}\right)=\sum_{m=1}^{m_{i}-1} \beta\left(m, m_{i}-m\right)
$$

Repeating the proof of (5.5) yields that $\mathbb{E}_{\varepsilon}^{e q}\left|H_{3112}(\omega(0))\right| \leq c_{1} K_{\varepsilon}^{1 / 2}|z|$. The term $H_{3111}$ is treated in the same fashion:

$$
\mathbb{E}_{\varepsilon}^{e q}\left|H_{3111}(\omega(0))\right| \leq c_{2} K_{\varepsilon}^{1 / 2}|z| \sum_{n, p} \sum_{m=1}^{n-1} \beta(m, n-m)(a(p)+a(m)) \lambda_{p} \lambda_{n} \leq c_{3} K_{\varepsilon}^{1 / 2}|z| .
$$

This completes the proof of (5.9).

We now turn to the proof of (5.10). The terms $H_{321}$ and $H_{322}$ are similar and both can be treated as (5.9). We only treat the latter. We certainly have that $\mathbb{E}_{\varepsilon}^{e q}\left|H_{321}(\omega(0))\right|$ is bounded by

$$
\begin{aligned}
& c_{1} \int K_{\varepsilon}^{1 / 2} \sum_{n, p} \sum_{m=1}^{n-1} \beta(m, n-m) \int V^{\varepsilon}(a-y ; m, n-m)\left|\hat{u}^{\varepsilon}(y-b ; m, p)\right| \\
& \quad 11(|y-b| \leq 1,|y|,|b| \leq l) d a d b d y \\
& =c_{1} \int K_{\varepsilon}^{1 / 2} \sum_{n, p} \sum_{m=1}^{n-1} \beta(m, n-m) \int\left|\hat{u}^{\varepsilon}(y-b ; m, p)\right| 11(|y-b| \leq 1,|y| \leq l) d b d y \\
& \leq c_{2} K_{\varepsilon}^{1 / 2}|z| \sum_{n, p} \sum_{m=1}^{n-1} \beta(m, n-m)(a(p)+a(m)) \lambda_{p} \lambda_{n} \leq c_{3} K_{\varepsilon}^{1 / 2}|z|,
\end{aligned}
$$

completing proof of (5.10).

Proof of (5.11) and (5.12).. We certainly have that the term $\mathbb{E}_{\varepsilon}^{e q}\left|H_{21}^{z}(\omega)\right|$ is bounded above by

$$
\begin{aligned}
& c_{1} \mathbb{E}_{\varepsilon}^{e q} K_{\varepsilon}^{-3 / 2} \sum_{i, j} \alpha\left(m_{i}, m_{j}\right) V_{\varepsilon}\left(x_{i}-x_{j} ; m_{i}, m_{j}\right)\left|u^{\varepsilon}\left(x_{i}-x_{j}+z ; m_{i}, m_{j}\right)\right| \mathbb{1}\left(\left|x_{i}\right| \leq l\right) \\
& \leq c_{2} K_{\varepsilon}^{-1 / 2} \sum_{m, n} \alpha(m, n) \alpha^{\prime}(m, n)\left|\log \frac{c_{2}|z|}{r(m, n)}\right| \mathbb{1}(\varepsilon r(m, n) \leq|z|) \lambda_{m} \lambda_{n} \\
& \quad+c_{2} K_{\varepsilon}^{1 / 2} \sum_{m, n} \alpha(m, n) \alpha^{\prime}(m, n) \lambda_{m} \lambda_{n} \mathbb{1}(\varepsilon r(m, n)>|z|) \\
& \leq c_{3} K_{\varepsilon}^{-1 / 2}|\log | z||+c_{3} K_{\varepsilon}^{-1 / 2} \sum_{m, n} \alpha(m, n) \alpha^{\prime}(m, n) \log r(m, n) \lambda_{m} \lambda_{n} \\
& \quad+c_{3} K_{\varepsilon}^{1 / 2} \sum_{n} a(n)^{2} \lambda_{n} \mathbb{1}(\varepsilon r(n)>|z|)
\end{aligned}
$$




$$
\begin{aligned}
& \leq c_{3} K_{\varepsilon}^{-1 / 2}|\log | z||+c_{4} K_{\varepsilon}^{-1 / 2} \sum_{n} a(n)^{2} \log n \lambda_{n}+c_{4} K_{\varepsilon}^{1 / 2} \sum_{n} a(n)^{2} \mathbb{1}(\varepsilon r(n)>|z|) \lambda_{n} \\
& \leq c_{3} K_{\varepsilon}^{-1 / 2}|\log | z||+c_{5} K_{\varepsilon}^{1 / 2}\left(\log \frac{|z|}{\varepsilon}\right)^{-1} \sum_{n} a(n)^{2} \log n \lambda_{n} \\
& \leq c_{3} K_{\varepsilon}^{-1 / 2}|\log | z||+c_{5} K_{\varepsilon}^{-1},
\end{aligned}
$$

where we used Lemma 6.1 for the first inequality. This completes the proof of (5.11).

Similarly the term $\mathbb{E}_{\varepsilon}^{e q}\left|H_{33}^{z}(\omega)\right|$ is bounded above by

$$
\begin{aligned}
& c_{1} \mathbb{E}_{\varepsilon}^{e q} \int K_{\varepsilon}^{-3 / 2} \sum_{i} \sum_{m=1}^{m_{i}-1} \beta\left(m, m_{i}-m\right) V^{\varepsilon}\left(x_{i}-y ; m, m_{i}-m\right) \\
& \quad\left|u^{\varepsilon}\left(x_{i}-y+z ; m, m_{i}-m\right)\right| 11\left(\left|x_{i}\right| \leq l\right) d y \\
& \leq c_{2} K_{\varepsilon}^{-1 / 2} \sum_{n} \sum_{m=1}^{n-1} \beta(m, n-m) \alpha^{\prime}(m, n-m) \\
& \quad\left|\log \frac{c_{2}|z|}{r(m, n-m)}\right| \mathbb{1}(\varepsilon r(m, n-m) \leq|z|) \lambda_{n} \\
& \quad+c_{2} K_{\varepsilon}^{1 / 2} \sum_{n} \sum_{m=1}^{n-1} \beta(m, n-m) \alpha^{\prime}(m, n-m) \mathbb{1}(\varepsilon r(m, n-m)>|z|) \lambda_{n} \\
& \leq c_{3} K_{\varepsilon}^{-1 / 2}|\log | z||+c_{3} K_{\varepsilon}^{-1} .
\end{aligned}
$$

This completes the proof of (5.12).

\section{Regularity of the Coagulation Term, Part II}

As we explained in Sect.4, one of the main steps of the proof of Theorem 3.1 is the replacement of the expression $V^{\varepsilon}(\cdot)$ in the collision term $H_{132}^{0}$ with a more manageable expression $W^{\varepsilon}(\cdot+z)$ for small $z$. Ultimately we average out $W^{\varepsilon}(\cdot+z)$ over $z$ and apply a CLT. For this to succeed, we need to make sure that we can afford a small $z$ which is as big as $|\log \varepsilon|^{-a}$ for some $a<1 / 2$. In Sect. 5, we used the auxiliary function $G$ in order to relate $H_{132}$ to $H_{13}^{z}$ provided that $|z|$ is of order $\delta(\varepsilon)=|\log \varepsilon|^{-\theta}$ for $\theta>1 / 2$. In this section, we would like to fill the gap by showing that in fact $z$ can be chosen so that $|z|$ is as large as $\delta^{\prime}(\varepsilon)=|\log \log \varepsilon|^{-\theta^{\prime}}$, provided that $\theta^{\prime} \in(0,1 / 2)$. To achieve this, we fix a $\theta \in(0,1 / 2)$ and set $\bar{H}_{13}(\omega)$ to be equal

$$
\int H_{13}^{z}(\omega) \zeta^{\delta(\varepsilon)}(z) d z=K_{\varepsilon}^{-3 / 2} \sum_{i, j} \alpha\left(m_{i}, m_{j}\right) \bar{W}^{\varepsilon}\left(x_{i}-x_{j} ; m_{i}, m_{j}\right) \hat{J}\left(x_{i}, m_{i}, x_{j}, m_{j}\right),
$$

where $\bar{W}^{\varepsilon}(a ; m, n)=\int W(a+z ; m, n) \zeta^{\delta(\varepsilon)}(z) d z$ and $W^{\varepsilon}$ was defined by (4.13). First observe that there exists a constant $c_{1}$ such that the function $\bar{W}^{\varepsilon}$ has a support that is contained in a ball of center 0 and radius $\delta(\varepsilon ; m, n)=c_{1} \delta(\varepsilon)+r(m, n) \varepsilon$. For our purposes, it is more convenient to assume that $r(m, n) \varepsilon \leq \delta(\varepsilon)$ so that for a constant $c_{2}$, the support $\bar{W}^{\varepsilon}$ is contained in a ball of center 0 and radius $c_{2} \delta(\varepsilon)$, with $c_{2}=c_{1}+1$, and that $\left|\bar{W}^{\varepsilon}\right| \leq c_{2} \delta(\varepsilon)^{-2}$. Such a restriction causes a small error. Indeed, if we set

$$
\bar{H}_{13}^{\prime}(\omega):=K_{\varepsilon}^{-3 / 2} \sum_{i, j} \alpha\left(m_{i}, m_{j}\right) \bar{W}^{\varepsilon}\left(x_{i}-x_{j} ; m_{i}, m_{j}\right) \bar{J}\left(x_{i}, m_{i}, x_{j}, m_{j}\right),
$$


with

$$
\bar{J}\left(x_{i}, m_{i}, x_{j}, m_{j}\right)=\tilde{J}\left(x_{i}, m_{i}, x_{j}, m_{j}\right) \mathbb{1}(r(m, n) \varepsilon \leq \delta(\varepsilon)),
$$

then

$$
\begin{aligned}
\mathbb{E}_{\varepsilon}^{e q}\left|\bar{H}_{13}^{\prime}(\omega)-\bar{H}_{13}(\omega)\right| & \leq c_{1} K_{\varepsilon}^{1 / 2} \sum_{m, n} \alpha(m, n) \lambda_{m} \lambda_{n} \mathbb{1}(r(m, n) \varepsilon>\delta(\varepsilon)) \\
& \leq c_{2} K_{\varepsilon}^{1 / 2} \sum_{n} a(n) \lambda_{n} \mathbb{1}(2 r(n) \varepsilon>\delta(\varepsilon)),
\end{aligned}
$$

which goes to 0 by our assumption (3.9).

Define $v^{\varepsilon}$ by

$$
v^{\varepsilon}(x ; m, n)=\frac{1}{2 \pi} \int \log |x-y| \bar{W}^{\varepsilon}(y ; m, n) d y .
$$

We then set

$$
G^{\prime}(\omega ; z)=G^{\prime}(\omega)=K_{\varepsilon}^{-3 / 2} \sum_{i, j} \hat{q}^{\varepsilon}\left(x_{i}-x_{j} ; m_{i}, m_{j}\right) \bar{J}\left(x_{i}, m_{i}, x_{j}, m_{j}\right),
$$

where $\hat{q}^{\varepsilon}(a ; m, n)=v^{\varepsilon}(a+z ; m, n) K(a+z)-v^{\varepsilon}(a ; m, n) K(a)$. We have

$$
G^{\prime}(\omega(t))=G^{\prime}(\omega(0))+\int_{0}^{t} \mathcal{A} G^{\prime}(\omega(s)) d s+M_{t}^{\prime},
$$

where $M_{t}^{\prime}$ is a martingale. Note that $G^{\prime}$ is very similar to $G$ of Sect. $5 ; \hat{u}$ is replaced with $\hat{q}$ and $\bar{J}$ is replaced with $\tilde{J}$. The latter difference has to do with the fact that now the function $K$ appears in the definition of $\hat{q}$ and we no longer need to multiply $\tilde{J}$ with a cut-off function. We write

$$
\mathcal{A} G^{\prime}=\mathcal{A}_{0} G^{\prime}+\mathcal{A}_{c} G^{\prime}+\mathcal{A}_{f} G^{\prime}=: H_{1}^{\prime}+H_{2}^{\prime}+H_{3}^{\prime} .
$$

We now study various terms which appeared on the right-hand side. We write

$$
H_{1}^{\prime}=H_{11}^{\prime}+H_{12}^{\prime}+H_{13}^{\prime}
$$

We do not repeat the definition of various $H^{\prime}$-expressions which all correspond to $H$ expressions of Sect. 5. However, since $v^{\varepsilon}$ satisfies (7.3), we have a different decomposition for $H_{13}^{\prime}$. The decomposition

$$
\begin{aligned}
\Delta \hat{q}^{\varepsilon}(a ; m, n)=\Delta & v^{\varepsilon}(a+z ; m, n) K(a+z)-\Delta v^{\varepsilon}(a ; m, n) K(a) \\
& +\nabla v^{\varepsilon}(a+z ; m, n) \cdot \nabla K(a+z)-\nabla v^{\varepsilon}(a ; m, n) \cdot \nabla K(a) \\
& +v^{\varepsilon}(a+z ; m, n) \Delta K(a+z)-v^{\varepsilon}(a ; m, n) \Delta K(a) \\
=: & q_{1}^{\varepsilon}(a ; m, n)+q_{2}^{\varepsilon}(a ; m, n)+q_{3}^{\varepsilon}(a ; m, n),
\end{aligned}
$$

results in a decomposition

$$
H_{13}^{\prime}=H_{131}^{\prime}+H_{132}^{\prime}+H_{133}^{\prime},
$$


where

$$
H_{13 r}^{\prime}=K_{\varepsilon}^{-3 / 2} \sum_{i, j} \hat{q}_{r}^{\varepsilon}\left(x_{i}-x_{j} ; m_{i}, m_{j}\right) \tilde{J}\left(x_{i}, m_{i}, x_{j}, m_{j}\right) .
$$

We may rewrite (7.5) as

$$
\begin{aligned}
\int_{0}^{t} H_{131}^{\prime}(\omega(s)) d s= & G(\omega(t))-G(\omega(0))-M_{t}^{\prime} \\
& -\int_{0}^{t}\left(H_{11}^{\prime}+H_{12}^{\prime}+H_{132}^{\prime}+H_{133}^{\prime}+H_{2}^{\prime}+H_{3}^{\prime}\right)(\omega(s)) d s .
\end{aligned}
$$

We are now ready to state the main result of this section.

Theorem 7.1. Assume that $\delta(\varepsilon)<|z|<1$. Then

$$
\mathbb{E}_{\varepsilon}^{e q}\left|\int_{0}^{t} H_{131}^{\prime}(\omega(s)) d s\right| \leq C_{0}(t+1)\left[|z|+K_{\varepsilon}^{-1 / 2}\right]|\log \delta(\varepsilon)|^{1 / 2} .
$$

Remark 7.1. With the aid of this theorem, we can readily improve the $z$-average from $|z|=O(\delta(\varepsilon))$ to $|z|=O\left(\delta^{\prime}(\varepsilon)\right)$. Indeed $H_{131}^{\prime}$ is given by

$$
K_{\varepsilon}^{-3 / 2} \sum_{i, j} \bar{W}^{\varepsilon}\left(x_{i}-x_{j}+z ; m_{i}, m_{j}\right) K\left(x_{i}-x_{j}+z\right) \bar{J}\left(x_{i}, m_{i}, x_{j}, m_{j}\right)-\bar{H}_{13}^{\prime},
$$

and by (7.2), the term $\bar{H}_{13}^{\prime}$ can be replaced with $\bar{H}_{13}$, for an error that goes to 0 as $\varepsilon \rightarrow 0$. From this and Theorem 7.1 we deduce that $\bar{H}_{13}=\int H_{13}^{z}(\omega) \zeta^{\delta(\varepsilon)}(z) d z$ can be replaced with

$$
K_{\varepsilon}^{-3 / 2} \sum_{i, j} \bar{W}^{\varepsilon}\left(x_{i}-x_{j}+z ; m_{i}, m_{j}\right) K\left(x_{i}-x_{j}+z\right) \tilde{J}\left(x_{i}, m_{i}, x_{j}, m_{j}\right),
$$

so long as $|z|=\delta^{\prime}(\varepsilon)$.

We establish Theorem 7.1 by examining various terms that appeared on the right-hand side of (7.6). Indeed we show

$$
\begin{gathered}
\mathbb{E}_{\varepsilon}^{e q}\left|G^{\prime}(\omega(t))\right| \leq C_{0}^{\prime}\left(K_{\varepsilon}^{-1 / 2}+|z|\right), \\
\mathbb{E}_{\varepsilon}^{e q}\left|\left(H_{11}^{\prime}+H_{133}^{\prime}\right)(\omega(s))\right| \leq C_{0}^{\prime}\left(K_{\varepsilon}^{-1 / 2}+|z|\right), \\
\mathbb{E}_{\varepsilon}^{e q}\left|\left(H_{12}^{\prime}+H_{132}^{\prime}\right)(\omega(s))\right| \leq C_{0}^{\prime} K_{\varepsilon}^{1 / 2}|z||\log | z||, \\
\mathbb{E}_{\varepsilon}^{e q}\left|H_{22}^{\prime}(\omega(s))\right| \leq C_{0}^{\prime}|z||\log \delta(\varepsilon)|^{1 / 2}, \\
\mathbb{E}_{\varepsilon}^{e q}\left|\left(H_{31}^{\prime}+H_{32}^{\prime}\right)(\omega(s))\right| \leq C_{0}^{\prime}\left(K_{\varepsilon}^{-1 / 2}+|z|\right), \\
\mathbb{E}_{\varepsilon}^{e q}\left|\left(H_{21}^{\prime}+H_{33}^{\prime}\right)(\omega(s))\right| \leq C_{0}^{\prime} K_{\varepsilon}^{-1 / 2}|\log \delta(\varepsilon)|, \\
\mathbb{E}_{\varepsilon}^{e q}\left[M_{t}^{\prime}\right]^{2} \leq C_{0}^{\prime} t\left(K_{\varepsilon}^{-1} \delta(\varepsilon)+|z|^{2}(\log |z|)^{2}\right) .
\end{gathered}
$$

To prepare for the proof of Theorem 7.1, we start with an elementary lemma. 
Lemma 7.1. Assume that $\int G(x, y, m, n) d x d y=0$, for every $m, n \in \mathbb{N}$. Then $\int Y^{2} d v_{\lambda}=Z_{1}+Z_{2}+Z_{3}$, where

$$
\begin{gathered}
Z_{1}=N(N-1)(N-2) \int \sum_{\substack{n_{1}, n_{2}, n_{3} \\
\lambda_{n_{1}} \lambda_{n_{2}} \lambda_{n_{3}} d y_{1} d y_{2} d y_{3},}} G\left(y_{1}, y_{2}, n_{1}, n_{2}\right) G\left(y_{1}, y_{3}, n_{1}, n_{3}\right) \\
Z_{2}=N(N-1)(N-2) \int \sum_{\substack{n_{1}, n_{2}, n_{3} \\
\lambda_{n_{1}} \lambda_{n_{2}} \lambda_{n_{3}} d y_{1} d y_{2} d y_{3},}} G\left(y_{1}, y_{2}, n_{1}, n_{2}\right) G\left(y_{3}, y_{2}, n_{3}, n_{1}\right) \\
Z_{3}=N(N-1) \int \sum_{n_{1}, n_{2}} G\left(y_{1}, y_{2}, n_{1}, n_{2}\right)^{2} d y_{1} d y_{2} \lambda_{n_{1}} \lambda_{n_{2}} .
\end{gathered}
$$

The straightforward proof of Lemma 7.1 is omitted. See also Lemma 3.3 of [R1] where a similar lemma is proved. As our next lemma we state some bounds on the function $v^{\varepsilon}$. The proof of this lemma is omitted because it is identical to the proof of Lemma 6.1.

Lemma 7.2. There exist positive constants $C_{1}$ and $C_{2}$ such that for all $x$,

$$
\begin{gathered}
\left|v^{\varepsilon}(x ; m, n)\right| \leq C_{1} \alpha^{\prime} \min \{1+|\log | x||,|\log \delta(\varepsilon)|\}, \\
\left|\nabla v^{\varepsilon}(x ; m, n)\right| \leq C_{1} \alpha^{\prime} \min \left\{|x|^{-1}, \delta(\varepsilon)^{-1}\right\},
\end{gathered}
$$

and for $|x| \geq 2|z|+C_{2} \delta(\varepsilon)$,

$$
\left|\nabla v^{\varepsilon}(x+z ; m, n)-\nabla v^{\varepsilon}(x ; m, n)\right| \leq C_{1} \gamma(\varepsilon ; m, n) \alpha^{\prime}|x|^{-2}|z| .
$$

Also,

$$
\begin{gathered}
\int\left|\nabla q^{\varepsilon}(a ; m, n)\right| d a \leq C_{1} \alpha^{\prime}, \\
\int\left|\hat{q}^{\varepsilon}(a ; m, n)\right| d a \leq C_{1} \alpha^{\prime}|z|, \\
\int\left|\nabla \hat{q}^{\varepsilon}(a ; m, n)\right| d x \leq C_{1} \alpha^{\prime}\{|z|[|\log (|z|+\delta(\varepsilon))|+1]+\delta(\varepsilon)\}, \\
\int q^{\varepsilon}(a ; m, n)^{2} d a \leq C_{1} \alpha^{\prime 2}, \\
\int\left|\nabla q^{\varepsilon}(a ; m, n)\right|^{2} d a \leq C_{1} \alpha^{\prime}|\log \delta(\varepsilon)| .
\end{gathered}
$$

Proof of (7.7) and (7.8). We only prove (7.7) because (7.8) can be proved by a verbatim argument. To apply Lemma 7.1, we need to check that for every $n_{1}$ and $n_{2}$,

$$
\int \hat{q}^{\varepsilon}\left(y_{1}-y_{2} ; n_{1}, n_{2}\right) \tilde{J}\left(y_{1}, n_{1}, y_{2}, n_{2}\right) d y_{1} d y_{2}=0 .
$$

We certainly have

$$
\int \hat{q}^{\varepsilon}\left(y_{1}-y_{2} ; n_{1}, n_{2}\right) J\left(y_{1}, n_{1}\right) d y_{1} d y_{2}=\int J\left(y, n_{1}\right) d y \int \hat{q}^{\varepsilon}\left(a ; n_{1}, n_{2}\right) d a=0 .
$$


The same is true if we replace $J\left(y_{1}, n_{1}\right)$ with $J\left(y_{1}, n_{1}+n_{2}\right)$. This completes the proof of (7.22). In view of Lemma 7.1,

$$
\mathbb{E}_{\varepsilon}^{e q} G^{\prime 2}(\omega(0))=\int G^{\prime 2}(\omega) \nu_{\lambda}(d \omega)=R_{1}+R_{2}+R_{3},
$$

with

$$
\begin{aligned}
R_{1}= & K_{\varepsilon}\left(K_{\varepsilon}-1\right)\left(K_{\varepsilon}-2\right) K_{\varepsilon}^{-3} \int \sum_{n_{1}, n_{2}, n_{3}} \hat{q}^{\varepsilon}\left(y_{1}-y_{2} ; n_{1}, n_{2}\right) \hat{q}^{\varepsilon}\left(y_{1}-y_{3} ; n_{1}, n_{3}\right) \\
& \bar{J}\left(y_{1}, n_{1}, y_{2}, n_{2}\right) \bar{J}\left(y_{1}, n_{1}, y_{3}, n_{3}\right) \lambda_{n_{1}} \lambda_{n_{2}} \lambda_{n_{3}} d y_{1} d y_{2} d y_{3}, \\
R_{3}= & K_{\varepsilon}\left(K_{\varepsilon}-1\right) K_{\varepsilon}^{-3} \int \sum_{n_{1}, n_{2}} \hat{q}^{\varepsilon}\left(y_{1}-y_{2} ; n_{1}, n_{2}\right)^{2} \bar{J}^{2}\left(y_{1}, n_{1}, y_{2}, n_{2}\right) \lambda_{n_{1}} \lambda_{n_{2}} d y_{1} d y_{2},
\end{aligned}
$$

and $R_{2}$ is given by an expression similar to $R_{1}$.

We start with bounding $R_{3}$. We certainly have

$$
R_{3} \leq c_{1} K_{\varepsilon}^{-1} \int \sum_{n_{1}, n_{2}} \lambda_{n_{1}} \lambda_{n_{2}}\left[q^{\varepsilon}\left(a ; n_{1}, n_{2}\right)^{2}+q^{\varepsilon}\left(a+z ; n_{1}, n_{2}\right)^{2}\right] d a .
$$

By Lemmas 7.2,

$$
R_{3} \leq c_{2} K_{\varepsilon}^{-1} \sum_{n_{1}, n_{2}} \alpha^{\prime}\left(n_{1}, n_{2}\right)^{2} \lambda_{n_{1}} \lambda_{n_{2}} \leq c_{3} K_{\varepsilon}^{-1} \sum_{n} a(n)^{2} \lambda_{n} \leq c_{4} K_{\varepsilon}^{-1} .
$$

We now turn to $R_{1}$. First observe that $R_{1} \leq R_{1}^{\prime}$, where

$$
R_{1}^{\prime}=c_{1} \sum_{n_{1}, n_{2}, n_{3}} \lambda_{n_{1}} \lambda_{n_{2}} \lambda_{n_{3}} \int\left|\hat{q}^{\varepsilon}\left(a ; n_{1}, n_{2}\right)\right| d a \int\left|\hat{q}^{\varepsilon}\left(a ; n_{1}, n_{3}\right)\right| d a .
$$

By Lemma 7.2 we deduce

$$
R_{11} \leq c_{1}|z|^{2} \sum_{n} a(n)^{2} \lambda_{n} \leq c_{2}|z|^{2}
$$

From this and (7.23) we deduce (7.7).

Proof of (7.9). Since $H_{12}^{\prime}$ is very similar to $H_{132}^{\prime}$, we only establish (7.9) for $H_{12}^{\prime}$. In view of Lemma 7.1,

$$
\mathbb{E}_{\varepsilon}^{e q} H_{12}^{\prime 2}(\omega(0))=R_{1}+R_{2}+R_{3},
$$

with

$$
\begin{aligned}
R_{1}= & K_{\varepsilon}\left(K_{\varepsilon}-1\right)\left(K_{\varepsilon}-2\right) K_{\varepsilon}^{-3} \int \sum_{n_{1}, n_{2}, n_{3}} \nabla \hat{q}^{\varepsilon}\left(y_{1}-y_{2} ; n_{1}, n_{2}\right) \cdot \nabla \hat{q}^{\varepsilon}\left(y_{1}-y_{3} ; n_{1}, n_{3}\right) \\
& \bar{J}\left(y_{1}, n_{1}, y_{2}, n_{2}\right) \bar{J}\left(y_{1}, n_{1}, y_{3}, n_{3}\right) \lambda_{n_{1}} \lambda_{n_{2}} \lambda_{n_{3}} d y_{1} d y_{2} d y_{3}, \\
R_{3}= & K_{\varepsilon}\left(K_{\varepsilon}-1\right) K_{\varepsilon}^{-3} \int \sum_{n_{1}, n_{2}}\left|\nabla \hat{q}^{\varepsilon}\left(y_{1}-y_{2} ; n_{1}, n_{2}\right)\right|^{2} \bar{J}^{2}\left(y_{1}, n_{1}, y_{2}, n_{2}\right) \lambda_{n_{1}} \lambda_{n_{2}} d y_{1} d y_{2},
\end{aligned}
$$

and $R_{2}$ is given by an expression similar to $R_{1}$. 
We start with bounding $R_{3}$. We certainly have

$$
R_{3} \leq c_{1} K_{\varepsilon}^{-1} \int \sum_{n_{1}, n_{2}} \lambda_{n_{1}} \lambda_{n_{2}}\left[\left|\nabla q^{\varepsilon}\left(a ; n_{1}, n_{2}\right)\right|^{2}+\left|\nabla q^{\varepsilon}\left(a+z ; n_{1}, n_{2}\right)\right|^{2}\right] d a .
$$

By Lemmas 7.2,

$$
\begin{aligned}
R_{3} & \leq c_{2}|\log \delta(\varepsilon)| K_{\varepsilon}^{-1} \sum_{n_{1}, n_{2}} \alpha^{\prime}\left(n_{1}, n_{2}\right)^{2} \lambda_{n_{1}} \lambda_{n_{2}} \\
& \leq c_{3} K_{\varepsilon}^{-1}|\log \delta(\varepsilon)| \sum_{n} a(n)^{2} \lambda_{n} \leq c_{4} K_{\varepsilon}^{-1}|\log \delta(\varepsilon)| .
\end{aligned}
$$

We now turn to $R_{1}$. First observe that $R_{1} \leq R_{1}^{\prime}$, where

$$
R_{1}^{\prime}=c_{1} \sum_{n_{1}, n_{2}, n_{3}} \lambda_{n_{1}} \lambda_{n_{2}} \lambda_{n_{3}} \int\left|\hat{q}^{\varepsilon}\left(a ; n_{1}, n_{2}\right)\right| d a \int\left|\hat{q}^{\varepsilon}\left(a ; n_{1}, n_{3}\right)\right| d a .
$$

By Lemma 7.2 we deduce

$$
R_{1} \leq c_{1}|z|^{2} \sum_{n_{1}, n_{2}, n_{3}} \alpha^{\prime}\left(n_{1}, n_{2}\right) \alpha^{\prime}\left(n_{1}, n_{3}\right) \lambda_{n_{1}} \lambda_{n_{2}} \lambda_{n_{3}} \leq c_{2}|z|^{2} .
$$

From this and (7.25) we deduce (7.9).

Proof of (7.11). As in the proof of (5.8) and (5.9), we have that $H_{31}^{\prime}=2 H_{311}^{\prime}, H_{311}^{\prime}=$ $H_{3111}^{\prime}-H_{3112}^{\prime}$, where

$$
H_{3112}^{\prime}(\omega)=\frac{1}{2} K_{\varepsilon}^{-3 / 2} \sum_{i, j} \beta^{\prime}\left(m_{i}\right) \hat{q}^{\varepsilon}\left(x_{i}-x_{j} ; m_{i}, m_{j}\right) \tilde{J}\left(x_{i}, m_{i}, x_{j}, m_{j}\right) .
$$

Repeating the proof of (7.9) yields

$$
\mathbb{E}_{e q}^{\varepsilon}\left[H_{3112}^{\prime}(\omega(s))\right]^{2} \leq c_{3}\left(|z|^{2}+K_{\varepsilon}^{-1}\right)
$$

The term $H_{3111}^{\prime}$ is handled in just the same way we handle $H_{32}^{\prime}$ below.

We now turn to $H_{32}^{\prime}$. The terms $H_{321}^{\prime}$ and $H_{322}^{\prime}$ are similar and both can be treated as (7.11). We only treat the latter. We apply Lemma 7.1 for $G\left(x_{i}, x_{j}, m_{i}, m_{j}\right)$ given by

$$
\frac{1}{2} \int \sum_{m=1}^{m_{j}-1} \beta\left(m, m_{j}-m\right) V^{\varepsilon}\left(x_{j}-y ; m, m_{j}-m\right) \hat{q}^{\varepsilon}\left(x_{i}-y ; m_{i}, m\right) \bar{J}\left(x_{i}, m_{i}, y, m\right) d y .
$$

As a result,

$$
\mathbb{E}_{\varepsilon}^{e q}\left[H_{322}(\omega(0))\right]^{2}=R_{1}+R_{2}+R_{3},
$$

with $R_{1}, R_{2}$, and $R_{3}$ corresponding to $Z_{1}, Z_{2}$, and $Z_{3}$ in Lemma 7.1.

We first treat $R_{3}$. For this term we need to bound $\left|G\left(x_{i}, x_{j}, m_{i}, m_{j}\right)\right|$. In this case we simply move the absolute value inside the summation and replace $\left|\hat{q}^{\varepsilon}\left(a ; m_{i}, m\right)\right|$ with a constant multiple of $\left|q^{\varepsilon}\left(a ; m_{i}, m\right)\right|+\left|q^{\varepsilon}\left(a+z ; m_{i}, m\right)\right|$. We then apply Lemma 6.1 to assert

$$
\left|G\left(x_{i}, x_{j}, m_{i}, m_{j}\right)\right| \leq S\left(x_{i}-x_{j}, m_{i}, m_{j}\right)+S\left(x_{i}-x_{j}+z, m_{i}, m_{j}\right),
$$


with $S(a, p, n)$ given by

$c_{2} \int \sum_{0}^{n-1} \beta(m, n-m) V^{\varepsilon}(y ; m, n-m) \alpha^{\prime}(p, m) \min \{|\log \delta(\varepsilon)|,|\log | a+y||\} \mathbb{1}(|a| \leq 2) d y$.

We have that there exists constants $c_{3}$ and $c_{4}$ such that

$S(a, p, n) \leq \begin{cases}c_{3} \sum_{0}^{n-1} \beta(m, n-m) \alpha^{\prime}(p, m)(|\log | a||+1), & \text { if }|a| \geq c_{4}(r(n) \varepsilon+\delta(\varepsilon)), \\ c_{3} \sum_{0}^{n-1} \beta(m, n-m) \alpha^{\prime}(p, m)|\log \delta(\varepsilon)|, & \text { otherwise. }\end{cases}$

From this we can readily deduce that $R_{3} \leq c_{5} K_{\varepsilon}^{-1}$, as in the proof of (7.11). (Note that $\sum_{0}^{n-1} \beta(m, n-m) \alpha^{\prime}(p, m) \leq(a(n)+a(p)) \beta^{\prime}(n)$ because by our choice, the function $a$ is non-decreasing.)

We now turn to $R_{1}$ and $R_{2}$. We certainly have that $\left|G\left(x_{i}, x_{j}, m_{i}, m_{j}\right)\right|$ is bounded above by

$$
\begin{gathered}
|z| \int_{0}^{1} \int \sum_{m=1}^{m_{j}-1} \beta\left(m, m_{j}-m\right) V^{\varepsilon}\left(x_{j}-y ; m, m_{j}-m\right) \\
\left|\nabla q^{\varepsilon}\left(x_{i}-y+t z ; m_{i}, m\right) \bar{J}\left(x_{i}, m_{i}, y, m\right)\right| d y d t .
\end{gathered}
$$

We then apply Lemma 7.2 to assert

$$
\left|G\left(x_{i}, x_{j}, m_{i}, m_{j}\right)\right| \leq|z| \int_{0}^{1} L\left(x_{i}-x_{j}+t z, m_{i}, m_{j}\right) d t,
$$

with $L(a, p, n)$ given by

$$
\begin{aligned}
& c_{5} \int \sum_{0}^{n-1} \beta(m, n-m) V^{\varepsilon}(y ; m, n-m) \alpha^{\prime}(p, m) \\
& \min \left\{\delta(\varepsilon)^{-1},|a+y|^{-1}\right\} \mathbb{1}(|a| \leq 2) d y .
\end{aligned}
$$

Again we can readily show

$$
L(a, n) \leq \begin{cases}c_{3} \sum_{0}^{n-1} \alpha^{\prime}(m, n) \beta(m, n-m)|a|^{-1}, & \text { if }|a| \geq c_{4}(r(n) \varepsilon+\delta(\varepsilon)), \\ c_{3} \sum_{0}^{n-1} \alpha^{\prime}(m, n) \beta(m, n-m) \delta(\varepsilon)^{-1}, & \text { otherwise. }\end{cases}
$$

Repeating the proof of (7.7) yields that $R_{1}+R_{2} \leq c_{7}|z|^{2}$, completing the proof of (7.11).

Proof of (7.12). We only establish (7.12) for $H_{21}^{\prime}$ because $H_{33}^{\prime}$ can be treated by an identical argument. Choose $c_{1}$ so that $V(a)=0$ if $|a|>c_{1}$. We certainly have that the expression $\mathbb{E}_{\mathcal{E}}^{e q}\left|H_{21}^{z}(\omega)\right|$ is bounded above by

$$
\begin{aligned}
& \mathbb{E}_{\varepsilon}^{e q} K_{\varepsilon}^{-3 / 2} \sum_{i, j} \alpha\left(m_{i}, m_{j}\right) V_{\varepsilon}\left(x_{i}-x_{j} ; m_{i}, m_{j}\right)\left|\hat{q}^{\varepsilon}\left(x_{i}-x_{j}+z ; m_{i}, m_{j}\right)\right| \\
& \quad \leq c_{1}|\log \delta(\varepsilon)| K_{\varepsilon}^{-1 / 2} \sum_{m, n} \alpha(m, n) \alpha^{\prime}(m, n) \lambda_{m} \lambda_{n} \\
& \quad \leq c_{2}|\log \delta(\varepsilon)| K_{\varepsilon}^{-1 / 2},
\end{aligned}
$$

where we used Lemma 6.1 for the first inequality. This completes the proof of (7.12). 
Proof of (7.10). We note that $H_{22}^{\prime}$ is a sum of eight terms $H_{22 i}^{\prime}, i=1, \ldots, 8$, and we establish (7.10) by showing the analogous bound for each $H_{22 i}^{\prime}$. Since all the eight terms can be treated in the same way, we only treat the sixth term which is given by

$$
\begin{aligned}
& H_{226}^{\prime}(\omega)=\frac{1}{2} K_{\varepsilon}^{-3 / 2} \sum_{i, j, k} \alpha\left(m_{i}, m_{j}\right) V_{\varepsilon}\left(x_{i}-x_{j} ; m_{i}, m_{j}\right) \\
& q^{\varepsilon}\left(x_{k}-x_{i} ; m_{k}, m_{i}\right) \bar{J}\left(x_{k}, m_{k}, x_{i}, m_{i}\right) .
\end{aligned}
$$

We note that $\bar{J}$ is a sum of 4 terms which yields a decomposition

$$
H_{226}^{\prime}=H_{2261}^{\prime}+H_{2262}^{\prime}-H_{2263}^{\prime}-H_{2264}^{\prime} \text {. }
$$

Again all the 4 terms can be treated in the same way, so we only treat $H_{2264}^{\prime}$ which is given by

$$
\frac{1}{2} K_{\varepsilon}^{-3 / 2} \sum_{i, j, k} \alpha\left(m_{i}, m_{j}\right) V_{\varepsilon}\left(x_{i}-x_{j} ; m_{i}, m_{j}\right) \tilde{q}^{\varepsilon}\left(x_{k}-x_{i} ; m_{k}, m_{i}\right) J\left(x_{i}, m_{i}\right),
$$

where $\tilde{q}\left(a ; m_{k}, m_{i}\right)=\hat{q}\left(a ; m_{k}, m_{i}\right) \mathbb{1}\left(r\left(m_{i}, m_{k}\right) \varepsilon \leq \delta(\varepsilon)\right)$. We use the elementary inequality $|a| \leq \delta+\delta^{-1} a^{2}$ to assert

$$
\left|H_{2264}^{\prime}\right| \leq H_{22641}^{\prime}+H_{22642}^{\prime}
$$

where $H_{22641}^{\prime}$ and $H_{22642}^{\prime}$ are respectively given by

$$
\begin{aligned}
& \frac{\delta}{2} K_{\varepsilon}^{-1} \sum_{i, j} \alpha\left(m_{i}, m_{j}\right) V_{\varepsilon}\left(x_{i}-x_{j} ; m_{i}, m_{j}\right)\left|J\left(x_{i}, m_{i}\right)\right| \\
& \quad \frac{\delta^{-1}}{2} K_{\varepsilon}^{-1} \sum_{i, j} \alpha\left(m_{i}, m_{j}\right) V_{\varepsilon}\left(x_{i}-x_{j} ; m_{i}, m_{j}\right)\left|J\left(x_{i}, m_{i}\right)\right| \\
& {\left[K_{\varepsilon}^{-1 / 2} \sum_{k} \tilde{q}^{\varepsilon}\left(x_{k}-x_{i} ; m_{k}, m_{i}\right)\right]^{2} .}
\end{aligned}
$$

Evidently, $\mathbb{E}_{e q}^{\varepsilon} H_{22641}^{\prime} \leq c_{1} \delta$, for some constant $c_{1}$. Moreover, by squaring the expression in the brackets, we learn that $H_{22642}^{\prime}=H_{226421}^{\prime}+H_{226422}^{\prime}$, where

$$
\begin{aligned}
H_{226421}^{\prime}= & \delta^{-1} K_{\varepsilon}^{-2} \sum_{i, j, k} \alpha\left(m_{i}, m_{j}\right) V_{\varepsilon}\left(x_{i}-x_{j} ; m_{i}, m_{j}\right)\left|J\left(x_{i}, m_{i}\right)\right| \tilde{q}^{\varepsilon}\left(x_{k}-x_{i} ; m_{k}, m_{i}\right)^{2}, \\
H_{226422}^{\prime}= & \delta^{-1} K_{\varepsilon}^{-2} \sum_{i, j, k \neq l} \alpha\left(m_{i}, m_{j}\right) V_{\varepsilon}\left(x_{i}-x_{j} ; m_{i}, m_{j}\right)\left|J\left(x_{i}, m_{i}\right)\right| \\
& \tilde{q}^{\varepsilon}\left(x_{k}-x_{i} ; m_{k}, m_{i}\right) \tilde{q}^{\varepsilon}\left(x_{l}-x_{i} ; m_{l}, m_{i}\right) .
\end{aligned}
$$

Because of our choice of $\tilde{q}$, we have that $\int \tilde{q}(a ; m, n) d a=0$. As a consequence,

$$
\mathbb{E}_{e q}^{\varepsilon} H_{226422}^{\prime}=0 \text {. }
$$


We certainly have that $\mathbb{E}_{e q}^{\varepsilon} H_{226421}^{\prime}$ is bounded above by

$$
\begin{aligned}
& c_{2} \delta^{-1} K_{\varepsilon} \sum_{n_{1}, n_{2}, n_{3}} \alpha\left(n_{1}, n_{2}\right) \lambda_{n_{1}} \lambda_{n_{2}} \lambda_{n_{3}} \int V_{\varepsilon}\left(a ; n_{1}, n_{2}\right) d a \int \hat{q}^{\varepsilon}\left(b ; n_{3}, n_{1}\right)^{2} d b \\
& =c_{2} \delta^{-1} \sum_{n_{1}, n_{2}, n_{3}} \alpha\left(n_{1}, n_{2}\right) \lambda_{n_{1}} \lambda_{n_{2}} \lambda_{n_{3}} \int \hat{q}^{\varepsilon}\left(b ; n_{3}, n_{1}\right)^{2} d b .
\end{aligned}
$$

On the other hand, by Lemma 7.2,

$$
\int \hat{q}^{\varepsilon}\left(b ; n_{3}, n_{1}\right)^{2} d b \leq\left.|z|^{2} \int \nabla q^{\varepsilon}\left(b ; n_{3}, n_{1}\right)\right|^{2} d b \leq c_{3} \alpha^{\prime}\left(n_{3}, n_{1}\right)^{2}|\log \delta(\varepsilon)||z|^{2} .
$$

As a result, $\mathbb{E}_{e q}^{\varepsilon} H_{226421}^{\prime}$ is bounded above by

$$
c_{4} \delta^{-1}|z|^{2}|\log \delta(\varepsilon)| \sum_{n_{1}, n_{2}, n_{3}} \alpha\left(n_{1}, n_{2}\right) \alpha^{\prime}\left(n_{3}, n_{1}\right)^{2} \lambda_{n_{1}} \lambda_{n_{2}} \lambda_{n_{3}} \leq c_{5} \delta^{-1}|z|^{2}|\log \delta(\varepsilon)| .
$$

In summary, from this (7.28), and (7.29) we deduce

$$
\mathbb{E}_{e q}^{\varepsilon}\left|H_{2264}^{\prime}\right| \leq c_{1} \delta+c_{5} \delta^{-1}|z|^{2}|\log \delta(\varepsilon)| .
$$

By choosing $\delta=|z||\log \delta(\varepsilon)|^{1 / 2}$ we deduce (7.10).

Proof of (7.13). As it is well-known,

$$
\mathbb{E}_{\varepsilon}^{e q}\left[M_{t}^{\prime}\right]^{2}=\mathbb{E}_{\varepsilon}^{e q} \int_{0}^{t}\left(\mathcal{A} G^{\prime}-2 G^{\prime} \mathcal{A} G^{\prime}\right)(\omega(s)) d s=t\left(Z_{1}+Z_{2}+Z_{3}\right),
$$

where

$$
\begin{aligned}
& Z_{1}=2 \mathbb{E}_{\varepsilon}^{e q}\left(\mathcal{A}_{0} G^{\prime}-2 G^{\prime} \mathcal{A}_{0} G^{\prime}\right)(\omega), \\
& Z_{2}=\mathbb{E}_{\varepsilon}^{e q}\left(\mathcal{A}_{c} G^{\prime}-2 G^{\prime} \mathcal{A}_{c} G^{\prime}\right)(\omega), \\
& Z_{3}=\mathbb{E}_{\varepsilon}^{e q}\left(\mathcal{A}_{f} G^{\prime}-2 G^{\prime} \mathcal{A}_{f} G^{\prime}\right)(\omega) .
\end{aligned}
$$

We start with bounding $Z_{1}$ :

$$
Z_{1}=K_{\varepsilon}^{-3} \mathbb{E}_{\varepsilon}^{e q} \sum_{i} d\left(m_{i}\right)\left|\nabla_{x_{i}} G^{\prime}(\omega)\right|^{2} \leq Z_{11}+Z_{12}+Z_{13}+Z_{14},
$$

where

$$
\begin{aligned}
& Z_{11}=4 K_{\varepsilon}^{-3} \mathbb{E}_{\varepsilon}^{e q} \sum_{i} d\left(m_{i}\right)\left|\sum_{j} \nabla \hat{q}^{\varepsilon}\left(x_{i}-x_{j} ; m_{i}, m_{j}\right) \bar{J}\left(x_{i}, m_{i}, x_{j}, m_{j}\right)\right|^{2}, \\
& Z_{12}=4 K_{\varepsilon}^{-3} \mathbb{E}_{\varepsilon}^{e q} \sum_{i} d\left(m_{i}\right)\left|\sum_{j} \hat{q}^{\varepsilon}\left(x_{i}-x_{j}, m_{i}, m_{j}\right) \nabla_{x_{i}} \bar{J}\left(x_{i}, m_{i}, x_{j}, m_{j}\right)\right|^{2} .
\end{aligned}
$$


The term $Z_{13}$ and $Z_{14}$ are given by a similar expression; $x_{i}$ and $x_{j}$ are swapped inside the absolute values. We only bound $Z_{11}$ because $Z_{11}$ involves $\nabla \hat{q}^{\varepsilon}$ which is more singular than $\hat{q}^{\varepsilon}$. The remaining $Z_{1 r}$ can be bounded in a similar way. Squaring yields

$$
\begin{aligned}
& Z_{11} \leq 4 K_{\varepsilon}^{-3} \mathbb{E}_{\varepsilon}^{e q} \sum_{i} d\left(m_{i}\right) \sum_{j \neq k} \nabla \hat{q}^{\varepsilon}\left(x_{i}-x_{j} ; m_{i}, m_{j}\right) \cdot \nabla \hat{q}^{\varepsilon}\left(x_{i}-x_{k} ; m_{i}, m_{k}\right) \\
& \quad \bar{J}\left(x_{i}, m_{i}, x_{j}, m_{j}\right) \bar{J}\left(x_{i}, m_{i}, x_{k}, m_{k}\right) \\
& \quad+4 K_{\varepsilon}^{-3} \mathbb{E}_{\varepsilon}^{e q} \sum_{i} d\left(m_{i}\right) \sum_{j}\left|\nabla \hat{q}^{\varepsilon}\left(x_{i}-x_{j} ; m_{i}, m_{j}\right)\right|^{2} \bar{J}\left(x_{i}, m_{i}, x_{j}, m_{j}\right)^{2} \\
& \quad=: Z_{111}+Z_{112} .
\end{aligned}
$$

Use Lemma 7.2 to deduce

$$
Z_{112} \leq c_{1} K_{\varepsilon}^{-1}|\log \delta(\varepsilon)| \sum_{n_{1}, n_{2}} d\left(n_{1}\right) \alpha^{\prime}\left(n_{1}, n_{2}\right)^{2} \lambda_{n_{1}} \lambda_{n_{2}} \leq c_{2} K_{\varepsilon}^{-1}|\log \delta(\varepsilon)| .
$$

We now turn to $Z_{111}$. By Lemma 7.2,

$$
\begin{aligned}
Z_{111} & \leq c_{1} \sum_{n_{1}, n_{2}, n_{3}} d\left(n_{1}\right)[|z||\log | z||+\delta(\varepsilon)]^{2} \alpha^{\prime}\left(n_{1}, n_{2}\right) \alpha^{\prime}\left(n_{1}, n_{3}\right) \lambda_{n_{1}} \lambda_{n_{2}} \lambda_{n_{3}} \\
& \leq c_{2}[|z||\log | z||+\delta(\varepsilon)]^{2} \leq 4 c_{2}[|z||\log | z||]^{2} .
\end{aligned}
$$

In summary

$$
Z_{1} \leq c_{3} K_{\varepsilon}^{-1}|\log \delta(\varepsilon)|+c_{3}[|z||\log | z||]^{2} .
$$

We now look at $Z_{2}$. We have

$$
\begin{aligned}
Z_{2}= & \frac{1}{2} K_{\varepsilon}^{-3} \mathbb{E}_{\varepsilon}^{e q} \sum_{i, j} \alpha\left(m_{i}, m_{j}\right) V_{\varepsilon}\left(x_{i}-x_{j} ; m_{i}, m_{j}\right) \\
& \times\left\{\sum_{k}\left[\Gamma_{i, j}(0)+\sum_{p=1}^{8} \Gamma_{i, j, k}(p)\right]\right\}^{2},
\end{aligned}
$$

where $\sum_{p=1}^{8} \Gamma_{i, j, k}(p)$ represents the eight terms that appeared in the definition of $\bar{H}_{22}^{\prime}$ and $\Gamma_{i, j}(0)=-\hat{q}^{\varepsilon}\left(x_{i}-x_{j} ; m_{i}, m_{j}\right) \bar{J}\left(x_{i}, m_{i}, x_{j}, m_{j}\right)$. An application of the inequality

$$
\left(\sum_{p=0}^{8} a_{p}\right)^{2} \leq 9 \sum_{p=0}^{8} a_{p}^{2}
$$

yields that $Z_{2}$ is bounded by

$$
\begin{aligned}
& \frac{9}{2} K_{\varepsilon}^{-3} \mathbb{E}_{\varepsilon}^{e q} \sum_{i, j} \alpha\left(m_{i}, m_{j}\right) V_{\varepsilon}\left(x_{i}-x_{j} ; m_{i}, m_{j}\right)\left[\Gamma_{i, j}(0)^{2}+\sum_{p=1}^{8}\left(\sum_{k} \Gamma_{i, j, k}(p)\right)^{2}\right] \\
& \quad=: \sum_{p=0}^{8} Z_{2 p}
\end{aligned}
$$


with for example,

$$
\begin{aligned}
Z_{28} \leq & \frac{9}{2} \mathbb{E}_{\varepsilon}^{e q} K_{\varepsilon}^{-3} \sum_{i, j} \alpha\left(m_{i}, m_{j}\right) V_{\varepsilon}\left(x_{i}-x_{j} ; m_{i}, m_{j}\right) \\
& {\left[\sum_{k} \hat{q}^{\varepsilon}\left(x_{k}-x_{j} ; m_{k}, m_{j}\right) \bar{J}\left(x_{k}, m_{k}, x_{j}, m_{j}\right)\right]^{2} . }
\end{aligned}
$$

We only treat $Z_{20}$ and $Z_{28}$ as the other terms $Z_{2 r}$ for $r=1, \ldots, 7$ can be treated as $Z_{28}$. We have $Z_{28}=Z_{281}+Z_{282}$, where

$$
\begin{aligned}
Z_{281}= & \frac{9}{2} \mathbb{E}_{\varepsilon}^{e q} K_{\varepsilon}^{-3} \sum_{i, j} V_{\varepsilon}\left(x_{i}-x_{j} ; m_{i}, m_{j}\right) \alpha\left(m_{i}, m_{j}\right) \\
& \sum_{k \neq l} \hat{q}^{\varepsilon}\left(x_{k}-x_{j} ; m_{k}, m_{j}\right) \hat{q}^{\varepsilon}\left(x_{l}-x_{j} ; m_{l}, m_{j}\right) \\
& \bar{J}\left(x_{k}, m_{k}, x_{j}, m_{j}\right) \bar{J}\left(x_{l}, m_{l}, x_{j}, m_{j}\right), \\
Z_{282}= & \frac{9}{2} \mathbb{E}_{\varepsilon}^{e q} K_{\varepsilon}^{-3} \sum_{i, j} V_{\varepsilon}\left(x_{i}-x_{j} ; m_{i}, m_{j}\right) \alpha\left(m_{i}, m_{j}\right) \\
& \sum_{k} \hat{q}^{\varepsilon}\left(x_{k}-x_{j} ; m_{k}, m_{j}\right)^{2} \bar{J}\left(x_{k}, m_{k}, x_{j}, m_{j}\right)^{2} .
\end{aligned}
$$

We start with the former

$$
\begin{gathered}
Z_{281} \leq c_{1} \sum_{n_{1}, n_{2}, n_{3}, n_{4}} \alpha\left(n_{1}, n_{2}\right) \lambda_{n_{1}} \lambda_{n_{2}} \lambda_{n_{3}} \lambda_{n_{4}} \int\left|\hat{q}^{\varepsilon}\left(a ; n_{3}, n_{2}\right)\right| d a \\
\int\left|\hat{q}^{\varepsilon}\left(a ; n_{4}, n_{2}\right)\right| d a \leq c_{2}|z|^{2} .
\end{gathered}
$$

As for $Z_{282}$ we have

$$
Z_{282} \leq c_{1} K_{\varepsilon}^{-1} \sum_{n_{1}, n_{2}, n_{3}} \alpha\left(n_{1}, n_{2}\right) \int \hat{q}^{\varepsilon}\left(a ; n_{3}, n_{2}\right)^{2} d a \lambda_{n_{1}} \lambda_{n_{2}} \lambda_{n_{3}} \leq c_{2} K_{\varepsilon}^{-1} .
$$

Finally

$$
Z_{280} \leq c_{1} K_{\varepsilon}^{-2} \sum_{n_{1}, n_{2}} \alpha\left(n_{1}, n_{2}\right) \lambda_{n_{1}} \lambda_{n_{2}} \int \hat{q}^{\varepsilon}\left(a ; n_{1}, n_{2}\right)^{2} d a \leq c_{2} K_{\varepsilon}^{-2} .
$$

In summary,

$$
Z_{2} \leq c_{1}\left(K_{\varepsilon}^{-1}+|z|^{2}\right) .
$$

We now turn to $Z_{3}$. We have

$$
\begin{aligned}
Z_{3}=\frac{1}{2} \mathbb{E}_{\varepsilon}^{e q} K_{\varepsilon}^{-3} \sum_{i} \sum_{m=1}^{m_{i}-1} \beta\left(m, m_{i}-m\right) \int V^{\varepsilon}\left(x_{i}-y ; m_{i}-m, m\right) \\
\\
{\left[\Gamma_{i}(y, m)+\sum_{p=1}^{4} \Gamma_{i}(p ; y, m)\right]^{2} d y, }
\end{aligned}
$$


where for example

$$
\begin{aligned}
\Gamma_{i}(y, m) & =\hat{q}^{\varepsilon}\left(x_{i}-y ; m, m_{i}-m\right) \bar{J}\left(x_{i}, m, m_{i}-m, y\right), \\
\Gamma_{i}(3 ; y, m) & =\sum_{j} \bar{q}^{\varepsilon}\left(y-x_{j} ; m, m_{j}\right) \bar{J}\left(y, m, x_{j}, m_{j}\right) .
\end{aligned}
$$

Again $Z_{3} \leq 5 \sum_{0}^{4} Z_{3 r}$ with for example

$$
Z_{33}=\frac{5}{2} \mathbb{E}_{\varepsilon}^{e q} K_{\varepsilon}^{-3} \sum_{i} \sum_{m=1}^{m_{i}-1} \beta\left(m, m_{i}-m\right) \int V^{\varepsilon}\left(x_{i}-y ; m_{i}-m, m\right) \Gamma_{i}(3 ; y, m)^{2} d y .
$$

We can now repeat the line of argument we had for $Z_{2}$ by squaring out $\Gamma_{i}$ and use Lemma 7.2 to get

$$
Z_{3} \leq c_{1}\left(K_{\varepsilon}^{-1}+|z|^{2}\right)
$$

From (7.30), (7.31) and (7.32) we deduce (7.13).

\section{Kinetic Limit}

In this section we establish the main claim of Theorem 3.1. We now state the martingale formulation of the Ornstein-Uhlenbeck diffusion which uniquely determines the solution of Eq. (3.13).

Definition 8.1. We say $\xi$ is a solution of (3.13) iffor any smooth function $J$ of compact support with $\int J=0$, the following processes are martingales:

$$
\begin{aligned}
M_{J}(t) & =M(t)=\xi(t, J)-\xi(0, J)-\int_{0}^{t} \Gamma \xi(s, J) d s, \\
N_{J}(t) & =N(t)=M(t)^{2}-t A(J) .
\end{aligned}
$$

Here $J=\left(J_{n}: n \in \mathbb{N}\right)$ with $J_{n}: \mathbb{R}^{d} \rightarrow \mathbb{R}$ and $\int J_{n}(x) d x=0, \Gamma=\Gamma_{0}+\Gamma_{c}+\Gamma_{f}$, and

$$
\begin{aligned}
\xi(t, J)= & \sum_{n} \xi_{n}\left(t, J_{n}\right), \\
\Gamma_{0} \xi(t, J)= & \sum_{n} d(n) \xi_{n}\left(t, \Delta_{x} J_{n}\right), \\
\Gamma_{c} \xi(t, J)= & \sum_{m, n} \hat{\alpha}(m, n) \lambda_{n} \xi_{n}\left(t, J_{n+m}-J_{n}-J_{m}\right), \\
\Gamma_{f}(\xi, J)= & \sum_{m, n} \hat{\beta}(m, n) \xi_{n}\left(t, J_{n}+J_{m}-J_{n+m}\right), \\
A(J)= & 2 \int \sum_{n} d(n) \lambda_{n}\left|\nabla_{x} J_{n}\right|^{2} d x+\frac{1}{2} \int \sum_{m, n} \hat{\alpha}(m, n) \lambda_{n} \lambda_{m}\left(J_{n+m}-J_{n}-J_{m}\right)^{2} d x \\
& +\frac{1}{2} \int \sum_{m, n} \hat{\beta}(m, n) \lambda_{n+m}\left(J_{n+m}-J_{n}-J_{m}\right)^{2} d x .
\end{aligned}
$$


We note that the last two terms in the definition of $A(J)$ are equal by the detailed balance assumption.

Ideally, we would like to show that the family $\mathcal{P}^{\varepsilon}$ is tight as $\varepsilon \rightarrow 0$ and that any limit point solves (3.13). Unfortunately we have not been able to establish the tightness and the difficulty comes from two error terms which go to 0 as $\varepsilon \rightarrow 0$ for each $t$. More precisely, let us define $\xi^{\prime}(t, J)=\xi(t, J)+\xi^{\prime \prime}(t, J)$, where

$$
\xi(t, J)=K_{\varepsilon}^{1 / 2} \sum_{i} J\left(x_{i}(t), m_{i}(t)\right),
$$

and $\xi^{\prime \prime}(t, J)=\frac{1}{2} \bar{G}_{\varepsilon}(\omega(t))$, with

$$
\bar{G}_{\varepsilon}(\omega)=\int G(\omega ; z) \zeta^{\delta(\varepsilon)}(z) d z+\iint G^{\prime}\left(\omega ; z_{2}-z_{1}\right) \zeta^{\delta^{\prime}(\varepsilon)}\left(z_{2}\right) \zeta^{\delta^{\prime}(\varepsilon)}\left(z_{1}\right) d z_{1} d z_{2} .
$$

Here $G$ and $G^{\prime}$ are as in (5.1) and (7.3), and $\zeta^{\delta}(a)=\delta^{-2} \zeta(a / \delta)$ with $\zeta$ a smooth non-negative symmetric function of compact support satisfying $\int \zeta(a) d a=1$. We take a countable dense subset $\mathcal{D}_{0}$ of smooth functions of compact support and write $\mathcal{H}=L^{1}([0, T] ; \mathbb{R})^{\mathcal{D}_{0}}$. The transformation $\omega(\cdot) \mapsto\left(\xi^{\prime}(\cdot, J): J \in \mathcal{D}_{0}\right)$ induces a probability measure $\hat{\mathcal{P}}^{\varepsilon}$ on $\mathcal{H}$. Let us write $\mathcal{P}$ for the distribution of a process $\xi$ which solves (3.13) and is subject to the following initial condition: $\xi(0, J)$ is a Gaussian random variable with variance

$$
\int \xi(0, J)^{2} \mathcal{P}(d \xi)=\sum_{n} \lambda_{n} \int J^{2}(x, n) d x .
$$

Note that $\xi(\cdot, J)$ is stationary under $\mathcal{P}$. Note also that $\mathcal{P}$ can be regarded as a probability measure on $\mathcal{H}$. It turns out that the tightness of the sequence $\hat{\mathcal{P}}^{\varepsilon}$ can be shown by standard arguments.

Theorem 8.1. The sequence $\hat{\mathcal{P}}^{\varepsilon}$ converges to $\mathcal{P}$ as $\varepsilon \rightarrow 0$. Moreover,

$$
\lim _{\varepsilon} \mathbb{E}_{\varepsilon}^{e q} \xi^{\prime \prime}(t, J)=0
$$

for every $t$.

We note that (8.2) is an immediate consequence of (5.5) and (7.7). The proof of the convergence of $\hat{\mathcal{P}}^{\varepsilon}$ is naturally divided into two steps. The first step is devoted to the proof of the tightness of the family $\hat{\mathcal{P}}^{\varepsilon}$. This step will be carried out in Sect.9. For the second step, we show that any limit point solves (3.13). This is a rather straight forward consequence of Theorem 8.2 below. This theorem is also the main ingredient for the proof of Theorem 3.1. We note that by a celebrated result of Holley and Stroock [HS], (3.13) has a unique solution in the sense of Definition 8.1.

Theorem 8.2. There exist martingales $M_{\varepsilon}$ and $N_{\varepsilon}$, and processes $\operatorname{Err}^{1, \varepsilon}$ and $\operatorname{Err}^{2, \varepsilon}$ such that

$$
\begin{gathered}
\xi^{\prime}(t, J)-\xi^{\prime}(0, J)-\int_{0}^{t} \Gamma \xi^{\prime}(s, J) d s=M_{\varepsilon}(t)+\operatorname{Err}^{1, \varepsilon}(t), \\
M_{\varepsilon}(t)^{2}-t A(J)=N_{\varepsilon}(t)+\operatorname{Err}^{2, \varepsilon}
\end{gathered}
$$


where $A(J)$ was defined in Definition 8.1, and

$$
\lim _{\varepsilon \rightarrow 0} \mathbb{E}_{\varepsilon}^{e q}\left|\operatorname{Err}^{1, \varepsilon}(t)\right|=\lim _{\varepsilon \rightarrow 0} \mathbb{E}_{\varepsilon}^{e q}\left|\operatorname{Err}^{2, \varepsilon}(t)\right|=0
$$

The proof of Theorem 8.2 is naturally divided into two parts.

Proof of (8.3). Step 1: Let us write $\bar{X}_{\varepsilon}(\omega)=X_{\varepsilon}(\omega)+\frac{1}{2} \bar{G}_{\varepsilon}(\omega)$, where $X_{\varepsilon}(\omega)$ and $\bar{G}^{\varepsilon}(\omega)$ were defined by (4.1) and (8.1) respectively. As it is a well-known fact for Markov processes, the following process is a martingale:

$$
\bar{M}_{\varepsilon}(t):=\bar{X}_{\varepsilon}(\omega(t))-\bar{X}_{\varepsilon}(\omega(0))-\int_{0}^{t} \mathcal{A} \bar{X}_{\varepsilon}(\omega(s)) d s .
$$

Note that by definition, $\bar{X}_{\varepsilon}(\omega(t))=\xi^{\prime}(t, J)$.

Let us study the term $\mathcal{A} \bar{X}_{\varepsilon}$. We certainly have

$$
\mathcal{A} \bar{X}_{\varepsilon}=\mathcal{A}_{0} X_{\varepsilon}+\mathcal{A}_{c} X_{\varepsilon}+\mathcal{A}_{f} X_{\varepsilon}+\frac{1}{2} \mathcal{A} \bar{G}_{\varepsilon}
$$

Note that the term $\mathcal{A} X_{\varepsilon}$ involves $\tilde{J}$ whereas $\mathcal{A} \bar{G}_{\varepsilon}$ involves $\hat{J}$. We replace $\tilde{J}$ of $\mathcal{A} X_{\varepsilon}$ with $\hat{J}$. This causes an error $\operatorname{Err}_{0}$ which is small because $\mathbb{E}_{\varepsilon}^{e q}\left|\operatorname{Err}_{0}\right|$ is bounded above by

$$
c_{1} K_{\varepsilon}^{1 / 2} \sum_{n, m} \alpha(m, n) \mathbb{1}\left(c_{0} \varepsilon r(m, n) \geq 1\right) \lambda_{m} \lambda_{n} \leq c_{2} K_{\varepsilon}^{1 / 2} \sum_{n} a(n) \mathbb{1}\left(2 c_{0} \varepsilon r(n) \geq 1\right) \lambda_{n} .
$$

As a result, we may use (3.9) to deduce

$$
\lim _{\varepsilon \rightarrow 0} \mathbb{E}_{\varepsilon}^{e q}\left|\operatorname{Err}_{0}\right|=0
$$

As a consequence of Theorems 5.1 and 7.1 (see Remark 7.1), we have

$\int_{0}^{t}\left(\mathcal{A}_{c} X_{\varepsilon}+\frac{1}{2} \mathcal{A} \bar{G}_{\varepsilon}\right)(\omega(s)) d s=\int_{0}^{t} Q_{\varepsilon}(\omega(s)) d s-\int_{0}^{t} H_{33}^{0}(\omega(s)) d s+\int_{0}^{t} \operatorname{Err}_{1} d s$, where $Q_{\varepsilon}(\omega)$ equals

$$
\begin{gathered}
\frac{1}{2} \int K_{\varepsilon}^{-3 / 2} \sum_{i, j} \alpha\left(m_{i}, m_{j}\right) \bar{W}^{\varepsilon}\left(x_{i}-x_{j}+z_{2}-z_{1} ; m_{i}, m_{j}\right) \\
\hat{J}\left(x_{i}, m_{i}, x_{j}, m_{j}\right) \zeta^{\delta}\left(z_{1}\right) \zeta^{\delta}\left(z_{2}\right) d z_{1} d z_{2},
\end{gathered}
$$

and

$$
\mathbb{E}_{\varepsilon}^{e q}\left|\operatorname{Err}_{1}\right| \leq c_{1}\left[K_{\varepsilon}^{1 / 2} \delta(\varepsilon)+K_{\varepsilon}^{-1 / 2}\right]|\log \delta(\varepsilon)|+c_{1}\left[\delta^{\prime}(\varepsilon)+K_{\varepsilon}^{-1 / 2}\right]|\log \delta(\varepsilon)|^{1 / 2},
$$

which goes to 0 in small $\varepsilon$ limit.

Step 2: Recall that the summation is over distinct $i$ and $j$ by our overall convention. However, one can readily check that if we allow $i=j$ in the summation, then the discrepancy is of order $O\left(K_{\varepsilon}^{-1 / 2}\right)$. Also, if we replace $\hat{J}$ with $\tilde{J}$, the error is of order $O(\tau(\varepsilon))$. The sum of these two errors is denoted by $E r r_{2}$, and we have

$$
\mathbb{E}_{\varepsilon}^{e q}\left|\operatorname{Err}_{2}\right| \leq c_{1}\left(K_{\varepsilon}^{-1 / 2}+\tau(\varepsilon)\right),
$$


which goes to 0 in small $\varepsilon$ limit. Because of the form of $\tilde{J}$, we may write

$$
Q_{\varepsilon}(\omega)=Q_{\varepsilon}^{1}+Q_{\varepsilon}^{2}-Q_{\varepsilon}^{3}-Q_{\varepsilon}^{4}+E r r_{2},
$$

where for example $Q_{\varepsilon}^{4}(\omega)$, given by

$$
\begin{gathered}
\frac{1}{2} \sum_{i, j} \int K_{\varepsilon}^{-3 / 2} \alpha\left(m_{i}, m_{j}\right) \bar{W}^{\varepsilon}\left(x_{i}-x_{j}+z_{2}-z_{1} ; m_{i}, m_{j}\right) \\
J\left(x_{j}, m_{j}\right) \zeta^{\delta^{\prime}(\varepsilon)}\left(z_{1}\right) \zeta^{\delta^{\prime}(\varepsilon)}\left(z_{2}\right) d z_{1} d z_{2},
\end{gathered}
$$

with the summation over all $i$ and $j$. We make a change of variables $x_{i}-z_{1}=a_{1}$, $x_{j}-z_{2}=a_{2}$ to write that $Q_{\varepsilon}^{4}(\omega)$ equals

$$
\begin{aligned}
\frac{1}{2} \sum_{i, j} K_{\varepsilon}^{-3 / 2} \int \alpha\left(m_{i}, m_{j}\right) \bar{W}^{\varepsilon}\left(a_{1}-a_{2} ; m_{i}, m_{j}\right) \\
J\left(x_{j}, m_{j}\right) \zeta^{\delta^{\prime}(\varepsilon)}\left(x_{i}-a_{1}\right) \zeta^{\delta^{\prime}(\varepsilon)}\left(x_{j}-a_{2}\right) d a_{1} d a_{2} \\
\quad=K_{\varepsilon}^{1 / 2} \sum_{m, n} \int \alpha(m, n) \bar{W}^{\varepsilon}\left(a_{1}-a_{2} ; m, n\right) f^{\varepsilon}\left(a_{1}, m ; \omega\right) f^{\varepsilon}\left(a_{2}, n ; \omega ; J\right) d a_{1} d a_{2}
\end{aligned}
$$

where

$$
\begin{aligned}
f^{\varepsilon}(a, m ; \omega) & =K_{\varepsilon}^{-1} \sum_{i} \zeta^{\delta^{\prime}(\varepsilon)}\left(x_{i}-a\right) \mathbb{1}\left(m_{i}=m\right), \\
f^{\varepsilon}(a, m ; \omega ; J) & =K_{\varepsilon}^{-1} \sum_{i} \zeta^{\delta^{\prime}(\varepsilon)}\left(x_{i}-a\right) J\left(x_{i}, m\right) \mathbb{1}\left(m_{i}=m\right) .
\end{aligned}
$$

We then have that $Q_{\varepsilon}^{4}(\omega)=Q_{\varepsilon}^{41}(\omega)+Q_{\varepsilon}^{42}(\omega)+\operatorname{Err}_{3}$, where

$$
\begin{aligned}
& Q_{\varepsilon}^{41}(\omega)=\frac{1}{2} \int K_{\varepsilon}^{1 / 2} \sum_{m, n} \alpha(m, n) \bar{W}^{\varepsilon}\left(a_{1}-a_{2}, m, n\right) \lambda_{m} f^{\varepsilon}\left(a_{2}, n ; \omega ; J\right) d a_{1} d a_{2}, \\
& Q_{\varepsilon}^{42}(\omega)=\frac{1}{2} \int K_{\varepsilon}^{1 / 2} \sum_{m, n} \alpha(m, n) \bar{W}^{\varepsilon}\left(a_{1}-a_{2} ; m, n\right) \lambda_{n} f^{\varepsilon}\left(a_{1}, m ; \omega\right) \bar{J}^{\varepsilon}\left(a_{2}, n\right) d a_{1} d a_{2},
\end{aligned}
$$

where $\bar{J}^{\varepsilon}(a, n)=\int \zeta^{\delta^{\prime}(\varepsilon)}(x-a) J(x, n) d x$ and $\operatorname{Err}_{3}$ is given by

$$
\begin{aligned}
\frac{1}{2} \int & K_{\varepsilon}^{1 / 2} \sum_{m, n} \alpha(m, n) \bar{W}^{\varepsilon}\left(a_{1}-a_{2} ; m, n\right) \\
& \left(f^{\varepsilon}\left(a_{1}, m ; \omega\right)-\lambda_{m}\right)\left(f^{\varepsilon}\left(a_{2}, n ; \omega ; J\right)-\lambda_{n} \bar{J}^{\varepsilon}\left(a_{2}, m\right)\right) d a_{1} d a_{2} \\
= & \frac{1}{2} \int K_{\varepsilon}^{-3 / 2} \sum_{i, j} \alpha\left(m_{i}, m_{j}\right) \bar{W}^{\varepsilon}\left(a_{1}-a_{2} ; m_{i}, m_{j}\right) \\
& \left(\zeta^{\delta^{\prime}(\varepsilon)}\left(x_{i}-a_{1}\right)-\lambda_{m_{i}}\right)\left(\zeta^{\delta^{\prime}(\varepsilon)}\left(x_{j}-a_{2}\right) J\left(x_{j}, m_{j}\right)-\lambda_{m_{j}} \bar{J}^{\varepsilon}\left(a_{2}, m_{j}\right)\right) d a_{1} d a_{2} .
\end{aligned}
$$

Here we have used the assumption $\int J=0$. We wish to show that $\operatorname{Err}_{3}$ is small. We first observe that we can write $\operatorname{Err}_{3}=E r r_{31}+\operatorname{Err}_{32}$, where $\operatorname{Err}_{31}$ is what we obtain 
by restricting the summation to indices $i \neq j$, and $\operatorname{Err}_{32}$ corresponds to the case $i=j$. It is not hard to show that $\operatorname{Err}_{32}$ is of order $O\left(K_{\varepsilon}^{-1 / 2}\right)$. On the other hand,

$$
\mathbb{E}_{\varepsilon}^{e q} \operatorname{Err}_{31}^{2} \leq c_{1} K_{\varepsilon}^{-1} \delta^{\prime}(\varepsilon)^{-2} \text {. }
$$

To see this, observe that $\operatorname{Err}_{31}^{2}$ equals

$$
\begin{aligned}
\frac{1}{2} \int & d a_{1} d a_{2} d b_{1} d b_{2} K_{\varepsilon}^{-3} \sum_{i, j, p, q} \alpha\left(m_{i}, m_{j}\right) \alpha\left(m_{p}, m_{q}\right) \\
& \bar{W}^{\varepsilon}\left(a_{1}-a_{2} ; m_{i}, m_{j}\right) \bar{W}^{\varepsilon}\left(b_{1}-b_{2} ; m_{p}, m_{q}\right) \\
& \left(\zeta^{\delta^{\prime}(\varepsilon)}\left(x_{i}-a_{1}\right)-\lambda_{m_{i}}\right)\left(\zeta^{\delta^{\prime}(\varepsilon)}\left(x_{p}-b_{1}\right)-\lambda_{m_{p}}\right) \\
& \left(\zeta^{\delta^{\prime}(\varepsilon)}\left(x_{j}-a_{2}\right) J\left(x_{j}, m_{j}\right)-\lambda_{m_{j}} \bar{J}^{\varepsilon}\left(a_{2}, m_{j}\right)\right) \\
& \left(\zeta^{\delta^{\prime}(\varepsilon)}\left(x_{q}-b_{2}\right) J\left(x_{q}, m_{q}\right)-\lambda_{m_{q}} \bar{J}^{\varepsilon}\left(b_{2}, m_{q}\right)\right) \\
= & : E_{1}+E_{2}+E_{3},
\end{aligned}
$$

where $E_{s}$ represents the above summation with $(i, j, p, q) \in I(s)$ with $I(1)$ corresponding to the cases $i \neq p, q$ or $p \neq i, j$ or $j \neq p, q$ or $q \neq i, j, I$ (2) corresponds to the case $i=p$ and $q=j$, and $I$ (3) corresponding to the case $i=q$ and $p=j$. (Recall that the summation in our expression for $\operatorname{Err}_{31}^{2}$ is over $i \neq j$ and $q \neq p$.) We can readily check

$$
\mathbb{E}_{\varepsilon}^{e q} E_{1}=0
$$

On the other hand $\mathbb{E}_{\varepsilon}^{e q} E_{2}$ equals

$$
\begin{aligned}
\frac{1}{2} & K_{\varepsilon}^{-1} \sum_{m, n} \int \bar{W}^{\varepsilon}\left(a_{1}-a_{2} ; m, n\right) \bar{W}^{\varepsilon}\left(b_{1}-b_{2} ; m, n\right) \alpha(m, n)^{2}\left(\lambda_{m} \gamma^{\delta^{\prime}(\varepsilon)}\left(a_{1}-b_{1}\right)-\lambda_{m}^{2}\right) \\
& \left(\lambda_{n} \zeta^{\delta^{\prime}(\varepsilon)}\left(y-a_{2}\right) \zeta^{\delta^{\prime}(\varepsilon)}\left(y-b_{2}\right) J^{2}(y, n)-\lambda_{n}^{2} \bar{J}^{\varepsilon}\left(a_{2}, n\right) \bar{J}^{\varepsilon}\left(b_{2}, n\right)\right) d y d a_{1} d a_{2} d b_{1} d b_{2} \\
& =\frac{1}{2}\left(E_{21}+E_{22}+E_{23}+E_{24}\right),
\end{aligned}
$$

where $\gamma^{\delta^{\prime}(\varepsilon)}(a)=\delta^{\prime}(\varepsilon)^{-2} \gamma\left(a / \delta^{\prime}(\varepsilon)\right)$, for $\gamma(a)=\int \zeta(a+b) \zeta(b) d b$, and $E_{2 r}$ for $r=1, \ldots, 4$, are given by

$$
\begin{aligned}
E_{21}= & K_{\varepsilon}^{-1} \sum_{m, n} \lambda_{m} \lambda_{n} \int \bar{W}^{\varepsilon}\left(a_{1}-a_{2} ; m, n\right) \bar{W}^{\varepsilon}\left(b_{1}-b_{2} ; m, n\right) \alpha(m, n)^{2} \gamma^{\delta^{\prime}(\varepsilon)}\left(a_{1}-b_{1}\right) \\
& \zeta^{\delta^{\prime}(\varepsilon)}\left(y-a_{2}\right) \zeta^{\delta^{\prime}(\varepsilon)}\left(y-b_{2}\right) J^{2}(y, n) d y d a_{1} d a_{2} d b_{1} d b_{2}, \\
E_{22}= & K_{\varepsilon}^{-1} \sum_{m, n} \lambda_{m}^{2} \lambda_{n} \int \bar{W}^{\varepsilon}\left(a_{1}-a_{2} ; m, n\right) \bar{W}^{\varepsilon}\left(b_{1}-b_{2} ; m, n\right) \alpha(m, n)^{2} \\
& \zeta^{\delta^{\prime}(\varepsilon)}\left(y-a_{2}\right) \zeta^{\delta^{\prime}(\varepsilon)}\left(y-b_{2}\right) J^{2}(y, n) d y d a_{1} d a_{2} d b_{1} d b_{2}, \\
E_{23}= & K_{\varepsilon}^{-1} \sum_{m, n} \lambda_{m} \lambda_{n}^{2} \int \bar{W}^{\varepsilon}\left(a_{1}-a_{2} ; m, n\right) \bar{W}^{\varepsilon}\left(b_{1}-b_{2} ; m, n\right) \alpha(m, n)^{2} \gamma^{\delta^{\prime}(\varepsilon)}\left(a_{1}-b_{1}\right) \\
& \bar{J}^{\varepsilon}\left(a_{2}, n\right) \bar{J}^{\varepsilon}\left(b_{2}, n\right) d y d a_{1} d a_{2} d b_{1} d b_{2}, \\
E_{24}= & K_{\varepsilon}^{-1} \sum_{m, n} \lambda_{m}^{2} \lambda_{n}^{2} \int \bar{W}^{\varepsilon}\left(a_{1}-a_{2} ; m, n\right) \bar{W}^{\varepsilon}\left(b_{1}-b_{2} ; m, n\right) \alpha(m, n)^{2} \\
& \bar{J}^{\varepsilon}\left(a_{2}, n\right) \bar{J}^{\varepsilon}\left(b_{2}, n\right) d y d a_{1} d a_{2} d b_{1} d b_{2} .
\end{aligned}
$$


We can readily see

$$
\left|E_{22}\right|+\left|E_{23}\right|+\left|E_{24}\right| \leq c_{1} K_{\varepsilon}^{-1}
$$

for a constant $c_{1}$. As for $E_{21}$ we have,

$$
\begin{aligned}
E_{21} \leq & c_{2} K_{\varepsilon}^{-1} \delta^{\prime}(\varepsilon)^{-2} \sum_{m, n} \lambda_{m} \lambda_{n} \int \bar{W}^{\varepsilon}\left(a_{1}-a_{2} ; m, n\right) \bar{W}^{\varepsilon} \\
& \left(b_{1}-b_{2} ; m, n\right) \alpha(m, n)^{2} \gamma^{\delta^{\prime}(\varepsilon)}\left(a_{1}-b_{1}\right) \\
& \zeta^{\delta^{\prime}(\varepsilon)}\left(y-a_{2}\right) J^{2}(y, n) d y d a_{1} d a_{2} d b_{1} d b_{2} \\
\leq & c_{3} K_{\varepsilon}^{-1} \delta^{\prime}(\varepsilon)^{-2} .
\end{aligned}
$$

Hence $\mathbb{E}_{\varepsilon}^{e q} E_{2} \leq c_{4} K_{\varepsilon}^{-1} \delta^{\prime}(\varepsilon)^{-2}$. Similarly $\mathbb{E}_{\varepsilon}^{e q} E_{3} \leq c_{2} K_{\varepsilon}^{-1 / 2} \delta^{\prime}(\varepsilon)^{-2}$ for a constant $c_{5}$. This and (8.8) yield (8.7).

Step 3: Note that $\hat{\alpha}=\alpha \lim _{\varepsilon \rightarrow 0} \int W^{\varepsilon}$ (this was proved in [HR1] as Theorem 3.2), and $\int \bar{W}^{\varepsilon}=\int W^{\varepsilon}$. Hence

$$
\begin{aligned}
Q_{\varepsilon}^{41}(\omega) & =\frac{1}{2} \int K_{\varepsilon}^{1 / 2} \sum_{m, n} \hat{\alpha}(m, n) \lambda_{m} f^{\varepsilon}\left(a_{2}, n ; \omega, J\right) d a_{2}+E r_{4} \\
& =\frac{1}{2} K_{\varepsilon}^{-1 / 2} \sum_{j} \sum_{m, n} \hat{\alpha}(m, n) \lambda_{m} J\left(x_{j}, n\right) \mathbb{1}\left(m_{j}=n\right)+E_{r} r_{41},
\end{aligned}
$$

where $E r r_{4}$ is the error we get by replacing $\int \bar{W}^{\varepsilon}=\int W^{\varepsilon}$ with its limit as $\varepsilon \rightarrow 0$. Since

$$
\mathbb{E}_{\varepsilon}^{e q}\left[K_{\varepsilon}^{-1 / 2} \sum_{j} \sum_{m, n} \hat{\alpha}(m, n) \lambda_{m} J\left(x_{j}, n\right) \mathbb{1}\left(m_{j}=n\right)\right] \leq c_{1},
$$

for a constant $c_{1}$ independent of $\varepsilon$, we deduce

$$
\lim _{\varepsilon \rightarrow 0} \mathbb{E}_{\varepsilon}^{e q}\left|E r r_{41}\right|^{2}=0
$$

Moreover, since $\bar{J}^{\varepsilon}(a, n)=\lambda_{n} J(a, n)+O\left(\delta^{\prime}(\varepsilon)\right)$, we have that $Q_{\varepsilon}^{42}(\omega)$ equals

$$
\begin{aligned}
& \frac{1}{2} \int K_{\varepsilon}^{1 / 2} \sum_{m, n} \alpha(m, n) \bar{W}^{\varepsilon}\left(a_{1}-a_{2} ; m, n\right) J\left(a_{2}, m\right) f^{\delta}\left(a_{1}, n ; \omega\right) d a_{1} d a_{2}+O(\delta) \\
& \quad=\frac{1}{2} \int K_{\varepsilon}^{1 / 2} \sum_{m, n} \hat{\alpha}(m, n) \lambda_{n} J\left(a_{1}, n\right) f^{\delta}\left(a_{1}, m ; \omega\right) d a_{1}+\operatorname{Err}_{42} \\
& \quad=\frac{1}{2} \int K_{\varepsilon}^{-1 / 2} \sum_{i} \sum_{m, n} \hat{\alpha}(m, n) \lambda_{n} J\left(x_{i}, n\right) \mathbb{1}\left(m_{i}=m\right)+\operatorname{Err}_{42}
\end{aligned}
$$

with

$$
\lim _{\varepsilon \rightarrow 0} \mathbb{E}_{\varepsilon}^{e q}\left|E r r_{42}\right|^{2}=0
$$


The terms $Q_{\varepsilon}^{j}$ for $j=1,2,3$ can be treated likewise. From (8.6), (8.7), (8.9), (8.10), (8.11), (8.12) and (8.2) we deduce

$$
\int_{0}^{t} Q_{\varepsilon}^{\delta}(\omega(s)) d s=\int_{0}^{t} \Gamma_{c} \xi(s, J) d s+\int_{0}^{t} \operatorname{Err}_{5} d s
$$

with

$$
\lim _{\varepsilon \rightarrow 0} \mathbb{E}_{\varepsilon}^{e q}\left|E r r_{5}\right|=0
$$

Step 4: We now study the term $H_{33}^{0}$. Recall

$$
\begin{aligned}
H_{33}^{0}(\omega)= & \frac{1}{2} K_{\varepsilon}^{-3 / 2} \sum_{i} \sum_{m=1}^{m_{i}-1} \beta\left(m, m_{i}-m\right) \\
& \int V^{\varepsilon}\left(x_{i}-y ; m, m_{i}-m\right) u^{\varepsilon}\left(x_{i}-y ; m, m_{i}-m\right) \hat{J}\left(x_{i}, m, y, m_{i}-m\right) d y .
\end{aligned}
$$

As we discussed in Step 1, we have replaced $\hat{J}$ with $\tilde{J}$ for an error which vanishes in small $\varepsilon$ limit. Moreover

$$
\tilde{J}\left(x_{i}, m, y, m_{i}-m\right)=\tilde{J}\left(x_{i}, m, x_{i}, m_{i}-m\right)+O\left(r\left(m, m_{i}-m\right) \varepsilon\right),
$$

whenever $V^{\varepsilon}\left(x_{i}-y ; m, m_{i}-m\right) \neq 0$. Hence $-H_{33}^{0}(\omega)+\mathcal{A}_{f} X_{\varepsilon}$ equals

$$
\begin{aligned}
& \frac{-1}{2} K_{\varepsilon}^{-1 / 2} \sum_{i} \sum_{m=1}^{m_{i}-1} \beta\left(m, m_{i}-m\right) \\
& \quad \times \int W^{\varepsilon}\left(x_{i}-y ; m, m_{i}-m\right) \tilde{J}\left(x_{i}, m, x_{i}, m_{i}-m\right) d y+\operatorname{Err}_{6} \\
& =\frac{-1}{2} K_{\varepsilon}^{-1 / 2} \sum_{i} \sum_{m=1}^{m_{i}-1} \hat{\beta}\left(m, m_{i}-m\right) \tilde{J}\left(x_{i}, m, x_{i}, m_{i}-m\right)+\operatorname{Err}_{7} \\
& =\Gamma_{f}(\xi, J)+\operatorname{Err}_{7},
\end{aligned}
$$

with

$$
\lim _{\varepsilon \rightarrow 0} \mathbb{E}_{\varepsilon}^{e q}\left|E r r_{7}\right|=0
$$

This is proved as in the previous step. For example, we have used

$$
\begin{aligned}
& \mathbb{E}_{\varepsilon}^{e q}\left[K_{\varepsilon}^{-1 / 2} \sum_{i} \sum_{m=1}^{m_{i}-1} \beta\left(m, m_{i}-m\right) \int W^{\varepsilon}\left(x_{i}-y ; m, m_{i}-m\right)\right. \\
& \left.\nabla \hat{J}\left(x_{i}, m, x_{i}+\theta\left(y-x_{i}\right), m_{i}-m\right) \cdot\left(y-x_{i}\right) d \theta d y\right]^{2}=O\left(\varepsilon^{2}\right),
\end{aligned}
$$

where $\nabla$ denotes the derivative with respect to the second spatial variable. 
Final Step: From (8.4-6) and (8.13-15) we deduce that the martingale $\bar{M}_{\varepsilon}(t)$ satisfies

$$
\begin{aligned}
\bar{M}_{\varepsilon}(t)= & \xi^{\prime}(t, J)-\xi^{\prime}(0, J)-\int_{0}^{t} \Gamma \xi(s, J) d s+\int_{0}^{t} \operatorname{Err}_{8}(\omega(s)) d s \\
= & \xi^{\prime}(t, J)-\xi^{\prime}(0, J)-\int_{0}^{t} \Gamma \xi^{\prime}(s, J) d s+\int_{0}^{t} \operatorname{Err}_{9}(\omega(s)) d s \\
& \lim _{\varepsilon \rightarrow 0} \mathbb{E}_{\varepsilon}^{e q}\left|\operatorname{Err}_{8}(\omega(0))\right|=\lim _{\varepsilon \rightarrow 0} \mathbb{E}_{\varepsilon}^{e q}\left|\operatorname{Err}_{9}(\omega(0))\right|=0 .
\end{aligned}
$$

Proof of (8.4). Step 1: Define $\hat{G}_{\varepsilon}(\omega)=\int G(\omega ; z) \zeta^{\delta(\varepsilon)}(z) d z$ and let us write $\hat{X}_{\varepsilon}(\omega)=$ $X_{\varepsilon}(\omega)+\frac{1}{2} \hat{G}_{\varepsilon}(\omega)$. Set,

$$
\hat{M}_{\varepsilon}(t):=\hat{X}_{\varepsilon}(\omega(t))-\hat{X}_{\varepsilon}(\omega(0))-\int_{0}^{t} \mathcal{A} \hat{X}_{\varepsilon}(\omega(s)) d s .
$$

Note that $\hat{M}_{\varepsilon}(t)=\bar{M}_{\varepsilon}(t)+M_{t}^{\prime}$, where $M_{t}^{\prime}$ was defined in Sect. 7. By (7.13),

$$
\lim _{\varepsilon \rightarrow 0} \mathbb{E}_{\varepsilon}^{e q}\left[\bar{M}_{\varepsilon}(t)-\hat{M}_{\varepsilon}(t)\right]^{2}=0 .
$$

As it is a well-known fact for Markov processes, the following process is also a martingale:

$$
\hat{N}_{\varepsilon}(t)=\hat{M}_{\varepsilon}(t)^{2}-\int_{0}^{t}\left(\mathcal{A}\left(\hat{X}_{\varepsilon}\right)^{2}-2 \hat{X}_{\varepsilon} \mathcal{A} \hat{X}_{\varepsilon}\right)(\omega(s)) d s .
$$

We certainly have

$$
A:=\mathcal{A}\left(\hat{X}_{\varepsilon}\right)^{2}-2 \hat{X}_{\varepsilon} \mathcal{A} \hat{X}_{\varepsilon}=A_{0}+A_{c}+A_{f},
$$

with

$$
A_{0}=2 K_{\varepsilon}^{-1} \sum_{i} d\left(m_{i}\right)\left|\nabla_{x} J\left(x_{i}, m_{i}\right)+B_{i}(\omega)\right|^{2},
$$

where

$$
\begin{aligned}
B_{i}(\omega)= & \frac{1}{2} K_{\varepsilon}^{-1} \nabla_{x_{i}} \sum_{j} \bar{u}^{\varepsilon}\left(x_{i}-x_{j} ; m_{i}, m_{j}\right) \hat{J}\left(x_{i}, m_{i}, x_{j}, m_{j}\right), \\
& +\frac{1}{2} K_{\varepsilon}^{-1} \nabla_{x_{i}} \sum_{j} \bar{u}^{\varepsilon}\left(x_{j}-x_{i} ; m_{j}, m_{i}\right) \hat{J}\left(x_{j}, m_{j}, x_{i}, m_{i}\right),
\end{aligned}
$$

with $\bar{u}^{\varepsilon}=\tilde{u}^{\varepsilon}-u^{\varepsilon}$, where $\tilde{u}^{\varepsilon}(a ; m, n)=\int u^{\varepsilon}(a+z ; m, n) \zeta^{\delta(\varepsilon)}(z) d z$. The exact form of $A_{c}$ and $A_{f}$ will be given in Steps 2 and 4 respectively. We have $B_{i}=B_{i 1}+B_{i 2}$, where 
$B_{i 1}(\omega)$ and $B_{i 2}(\omega)$ are given by

$$
\begin{gathered}
\frac{1}{2} K_{\varepsilon}^{-1} \sum_{j}\left(\bar{u}_{x}^{\varepsilon}\left(x_{i}-x_{j} ; m_{i}, m_{j}\right) \hat{J}\left(x_{j}, m_{j}, x_{i}, m_{i}\right)\right. \\
\left.-\bar{u}_{x}^{\varepsilon}\left(x_{j}-x_{i} ; m_{j}, m_{i}\right) \hat{J}\left(x_{i}, m_{i}, x_{j}, m_{j}\right)\right), \\
\frac{1}{2} K_{\varepsilon}^{-1} \sum_{j}\left(\bar{u}^{\varepsilon}\left(x_{i}-x_{j} ; m_{i}, m_{j}\right) \hat{J}_{x}\left(x_{i}, m_{i}, x_{j}, m_{j}\right)\right. \\
\left.-\bar{u}^{\varepsilon}\left(x_{j}-x_{i} ; m_{j}, m_{i}\right) \hat{J}_{y}\left(x_{j}, m_{j}, x_{i}, m_{i}\right)\right),
\end{gathered}
$$

respectively. We have

$$
A_{0}=2 A_{00}+2 A_{01}+2 A_{02},
$$

with

$$
\begin{aligned}
& A_{00}=K_{\varepsilon}^{-1} \sum_{i} d\left(m_{i}\right)\left|\nabla_{x} J\left(x_{i}, m_{i}\right)\right|^{2}, \\
& A_{01}=2 K_{\varepsilon}^{-1} \sum_{i} d\left(m_{i}\right)\left(B_{i 1}(\omega)+B_{i 2}(\omega)\right) \cdot \nabla_{x} J\left(x_{i}, m_{i}\right), \\
& A_{02}=K_{\varepsilon}^{-1} \sum_{i} d\left(m_{i}\right)\left(B_{i 1}(\omega)+B_{i 2}(\omega)\right)^{2} .
\end{aligned}
$$

We first show that the term $A_{01}$ is small. Note that $\left|A_{01}\right| \leq A_{011}+A_{012}$, for

$$
A_{01 r}=c_{1} K_{\varepsilon}^{-1} \sum_{i} d\left(m_{i}\right)\left|B_{i r}(\omega)\right|,
$$

with $c_{1}=2\|\nabla J\|_{\infty}$. By Lemma 6.1,

$$
\begin{aligned}
& \mathbb{E}_{e q}^{\varepsilon} A_{011} \leq c_{2} \sum_{m, n} \lambda_{m} \lambda_{n} \int\left|\nabla \bar{u}^{\varepsilon}(a ; m, n)\right| d a \leq c_{3} \delta(\varepsilon) \log \delta(\varepsilon), \\
& \mathbb{E}_{e q}^{\varepsilon} A_{012} \leq c_{2} \sum_{m, n} \lambda_{m} \lambda_{n} \int_{|a| \leq 1}\left|\bar{u}^{\varepsilon}(a ; m, n)\right| d a \leq c_{3} \delta(\varepsilon),
\end{aligned}
$$

as in the proof of (5.5) and (5.7). As a result,

$$
\left|A_{01}\right| \leq 2 c_{3} \delta(\varepsilon) \log \delta(\varepsilon) .
$$

We now turn to $A_{02}$. We may write $A_{02}=A_{021}+A_{022}+A_{023}$, with

$$
\begin{aligned}
& A_{021}=K_{\varepsilon}^{-1} \sum_{i} d\left(m_{i}\right) B_{i 1}^{2}, \\
& A_{022}=K_{\varepsilon}^{-1} \sum_{i} d\left(m_{i}\right) B_{i 2}^{2}, \\
& A_{023}=2 K_{\varepsilon}^{-1} \sum_{i} d\left(m_{i}\right) B_{i 1} B_{i 2} .
\end{aligned}
$$


We now use Lemma 6.2 to show that $A_{022}$ and $A_{023}$ are small. Indeed after squaring,

$$
\begin{aligned}
\mathbb{E}_{e q}^{\varepsilon} A_{022} & \leq c_{4} \mathbb{E}_{e q}^{\varepsilon} K_{\varepsilon}^{-3} \sum_{i, j, k} d\left(m_{i}\right)\left|\bar{u}^{\varepsilon}\left(x_{i}-x_{j} ; m_{i}, m_{j}\right)\right|\left|\bar{u}^{\varepsilon}\left(x_{i}-x_{k} ; m_{i}, m_{k}\right)\right| \\
& =A_{0221}+A_{0222},
\end{aligned}
$$

where $A_{0221}$ and $A_{0222}$ correspond to the cases $k \neq j$ and $k=j$ respectively. By Lemma 6.2 and (3.10),

$$
\begin{aligned}
& \mathbb{E}_{e q}^{\varepsilon} A_{0221} \leq c_{5} \sum_{m, n, p} \lambda_{m} \lambda_{n} \lambda_{p} \int_{|a| \leq 1}\left|\bar{u}^{\varepsilon}(a ; m, n)\right| d a \int_{|a| \leq 1}\left|\bar{u}^{\varepsilon}(a ; m, p)\right| d a \leq c_{6} \delta(\varepsilon)^{2}, \\
& \mathbb{E}_{e q}^{\varepsilon} A_{0222} \leq c_{5} K_{\varepsilon}^{-1} \sum_{m, n} \lambda_{m} \lambda_{n} \int_{|a| \leq 1}\left|\bar{u}^{\varepsilon}(a ; m, n)\right|^{2} d a \leq c_{6} K_{\varepsilon}^{-1} .
\end{aligned}
$$

In the same fashion,

$$
\begin{aligned}
\mathbb{E}_{e q}^{\varepsilon} A_{023} & \leq c_{7} \mathbb{E}_{e q}^{\varepsilon} K_{\varepsilon}^{-3} \sum_{i, j, k} d\left(m_{i}\right)\left|\bar{u}^{\varepsilon}\left(x_{i}-x_{j} ; m_{i}, m_{j}\right)\right|\left|\nabla \bar{u}^{\varepsilon}\left(x_{i}-x_{k} ; m_{i}, m_{k}\right)\right| \\
& =A_{0231}+A_{0232}
\end{aligned}
$$

where $A_{0231}$ and $A_{0232}$ correspond to the cases $k \neq j$ and $k=j$ respectively. By Lemma 6.2 and (3.10),

$$
\begin{aligned}
\mathbb{E}_{e q}^{\varepsilon} A_{0231} \leq & c_{8} \sum_{m, n, p} \lambda_{m} \lambda_{n} \lambda_{p} \int_{|a| \leq 1}\left|\bar{u}^{\varepsilon}(a ; m, n)\right| d a \\
& \times \int_{|a| \leq 1}\left|\nabla \bar{u}^{\varepsilon}(a ; m, p)\right| d a \leq c_{9} \delta(\varepsilon)^{2}|\log \delta(\varepsilon)|, \\
\mathbb{E}_{e q}^{\varepsilon} A_{0232} \leq & c_{10} K_{\varepsilon}^{-1} \sum_{m, n} \lambda_{m} \lambda_{n} \\
& \times \int_{|a| \leq 1}\left|\bar{u}^{\varepsilon}(a ; m, n)\right|\left|\nabla \bar{u}^{\varepsilon}(a ; m, n)\right| d a \\
\leq & c_{10} K_{\varepsilon}^{-1} \sum_{m, n} \lambda_{m} \lambda_{n}\left(\int_{|a| \leq 1}\left|\bar{u}^{\varepsilon}(a ; m, n)\right|^{2} d a\right)^{1 / 2} \\
& \times\left(\int_{|a| \leq 1}\left|\nabla \bar{u}^{\varepsilon}(a ; m, n)\right|^{2} d a\right)^{1 / 2} \\
\leq & c_{11} K_{\varepsilon}^{-1} \delta(\varepsilon) K_{\varepsilon}^{1 / 2} K_{\varepsilon}^{1 / 2}=c_{11} \delta(\varepsilon) .
\end{aligned}
$$

As a result,

$$
\left|A_{022}+A_{023}\right| \leq c_{12}\left(\delta(\varepsilon)+K_{\varepsilon}^{-1}\right) .
$$

We now concentrate on $A_{021}$. First observe that since $V$ and $\zeta$ are symmetric, we learn that $\bar{u}^{\varepsilon}$ is symmetric. From this and symmetry of $\tilde{J}$ and $K$ we learn

$$
B_{i 1}=K_{\varepsilon}^{-1} \sum_{j} \bar{u}_{x}^{\varepsilon}\left(x_{i}-x_{j} ; m_{i}, m_{j}\right) \hat{J}\left(x_{i}, m_{i}, x_{j}, m_{j}\right) .
$$


After squaring, we obtain $A_{021}=A_{0211}+A_{0212}$, where

$$
\begin{aligned}
A_{0211}= & 2 K_{\varepsilon}^{-3} \sum_{i, j, k} d\left(m_{i}\right) \bar{u}_{x}^{\varepsilon}\left(x_{i}-x_{j} ; m_{i}, m_{j}\right) \cdot \bar{u}_{x}^{\varepsilon}\left(x_{i}-x_{k} ; m_{i}, m_{k}\right) \\
& \hat{J}\left(x_{i}, m_{i}, x_{j}, m_{j}\right) \hat{J}\left(x_{i}, m_{i}, x_{k}, m_{k}\right), \\
A_{0212}= & K_{\varepsilon}^{-3} \sum_{i, j} d\left(m_{i}\right)\left|\bar{u}_{x}^{\varepsilon}\left(x_{i}-x_{j} ; m_{i}, m_{j}\right)\right|^{2} \hat{J}\left(x_{i}, m_{i}, x_{j}, m_{j}\right)^{2},
\end{aligned}
$$

where $j \neq k$ in $A_{0211}$. Using Lemma 6.1 we deduce

$$
\begin{aligned}
& \mathbb{E}_{e q}^{\varepsilon}\left|A_{0211}\right| \leq c_{1} \sum_{m, n, p} \lambda_{m} \lambda_{n} \lambda_{p} \int_{|a| \leq 1}\left|\nabla \bar{u}^{\varepsilon}(a ; m, n)\right| d a \\
& \quad \times \int_{|a| \leq 1}\left|\nabla \bar{u}^{\varepsilon}(a ; m, p)\right| d a \leq c_{2} \delta(\varepsilon)^{2}(\log \delta(\varepsilon))^{2} .
\end{aligned}
$$

As for $A_{0212}$, we first write, $A_{0212}=A_{02121}+A_{02122}+A_{02123}$, where

$$
\begin{aligned}
& A_{02121}=K_{\varepsilon}^{-3} \sum_{i, j} d\left(m_{i}\right)\left|u_{x}^{\varepsilon}\left(x_{i}-x_{j} ; m_{i}, m_{j}\right)\right|^{2} \hat{J}\left(x_{i}, m_{i}, x_{j}, m_{j}\right)^{2}, \\
& A_{02122}=K_{\varepsilon}^{-3} \sum_{i, j} d\left(m_{i}\right)\left|\tilde{u}_{x}^{\varepsilon}\left(x_{i}-x_{j} ; m_{i}, m_{j}\right)\right|^{2} \hat{J}\left(x_{i}, m_{i}, x_{j}, m_{j}\right)^{2}, \\
& A_{02123}=-2 K_{\varepsilon}^{-3} \sum_{i, j} d\left(m_{i}\right)\left(\tilde{u}_{x}^{\varepsilon} \cdot u_{x}^{\varepsilon}\right)\left(x_{i}-x_{j} ; m_{i}, m_{j}\right) \hat{J}\left(x_{i}, m_{i}, x_{j}, m_{j}\right)^{2} .
\end{aligned}
$$

We now argue that $A_{02122}$ and $A_{02123}$ are small. To see this, first observe that by Lemma 6.2,

$$
\begin{aligned}
\left|\nabla \tilde{u}^{\varepsilon}(a ; m, n)\right| & =\left|\iint \nabla u^{\varepsilon}(a+z ; m, n) \zeta^{\delta(\varepsilon)}(z) d z\right| \\
& \leq c_{3} \delta(\varepsilon)^{-2} \int_{|z| \leq c_{3} \delta(\varepsilon)}\left|\nabla u^{\varepsilon}(a+z ; m, n)\right| d z \\
& \leq c_{4} \delta(\varepsilon)^{-2} \alpha^{\prime}(m, n) \int_{|z| \leq c_{3} \delta(\varepsilon)}|a+z|^{-1} d z \\
& \leq c_{5} \alpha^{\prime}(m, n) \min \left\{|a|^{-1}, \delta(\varepsilon)^{-1}\right\}
\end{aligned}
$$

As a result,

$$
\begin{gathered}
\int_{|a| \leq 1}\left|\nabla \tilde{u}^{\varepsilon}(a ; m, n)\right|^{2} d a \leq c_{6} \alpha^{\prime}(m, n)^{2}|\log \delta(\varepsilon)|, \\
\int_{|a| \leq 1}\left|\nabla \tilde{u}^{\varepsilon}(a ; m, n) \cdot \nabla u^{\varepsilon}(a ; m, n)\right| d a \leq c_{6} \alpha^{\prime}(m, n)^{2}|\log \delta(\varepsilon)|^{1 / 2}|\log \varepsilon|^{1 / 2} .
\end{gathered}
$$

From this we learn

$$
\left|A_{02122}\right| \leq c_{7} \alpha^{\prime}(m, n)^{2} \frac{|\log \delta(\varepsilon)|}{|\log \varepsilon|}, \quad\left|A_{02123}\right| \leq c_{7} \alpha^{\prime}(m, n)^{2} \frac{|\log \delta(\varepsilon)|^{1 / 2}}{|\log \varepsilon|^{1 / 2}} .
$$


From (8.18)-(8.21) we deduce that $A_{0}=2 A_{00}+2 A_{02121}+E r r_{1}$, with

$$
\mathbb{E}_{\varepsilon}^{e q}\left|\operatorname{Err}_{1}\right| \leq c\left(\delta(\varepsilon)|\log \delta(\varepsilon)|+|\log \varepsilon|^{-1 / 2}|\log \delta(\varepsilon)|^{1 / 2}\right) .
$$

Furthermore, if we pick a small $\delta>0$ and write $A_{02121}=A_{021211}+A_{021212}$, with

$$
\begin{aligned}
& A_{021211}=K_{\varepsilon}^{-3} \sum_{i, j} d\left(m_{i}\right)\left|u_{x}^{\varepsilon}\left(x_{i}-x_{j} ; m_{i}, m_{j}\right)\right|^{2} \hat{J}\left(x_{i}, m_{i}, x_{j}, m_{j}\right)^{2} \mathbb{1}\left(\left|x_{i}-x_{j}\right| \leq \delta\right), \\
& A_{021212}=K_{\varepsilon}^{-3} \sum_{i, j} d\left(m_{i}\right)\left|u_{x}^{\varepsilon}\left(x_{i}-x_{j} ; m_{i}, m_{j}\right)\right|^{2} \hat{J}\left(x_{i}, m_{i}, x_{j}, m_{j}\right)^{2} \mathbb{1}\left(\left|x_{i}-x_{j}\right|>\delta\right),
\end{aligned}
$$

then we have that $\mathbb{E}_{\varepsilon}^{e q}\left|A_{021212}\right| \leq K_{\varepsilon}^{-1} \delta^{-2}$, and $A_{021211}=A_{0212111}+A_{0212112}$, where in the first term we replace the $x_{j}$ argument of $\hat{J}$ with $x_{i}$, and the second term is the error caused by such a replacement. More precisely,

$$
\begin{gathered}
A_{0212111}=K_{\varepsilon}^{-3} \sum_{i, j} d\left(m_{i}\right)\left|u_{x}^{\varepsilon}\left(x_{i}-x_{j} ; m_{i}, m_{j}\right)\right|^{2} \\
\tilde{J}\left(x_{i}, m_{i}, x_{i}, m_{j}\right)^{2} \mathbb{1 1}\left(\left|x_{i}-x_{j}\right| \leq \delta\right), \\
\mathbb{E}_{\varepsilon}^{e q}\left|A_{0212112}\right| \leq K_{\varepsilon}^{-1} \delta c_{1} \sum_{m, n} \lambda_{m} \lambda_{n} \int_{|a| \leq \delta}\left|\nabla u^{\varepsilon}(a ; m, n)\right|^{2} d a \leq c_{2} \delta,
\end{gathered}
$$

where we used the smoothness of $J$ for the first inequality and (6.8) for the second inequality. Now that we have replaced $x_{j}$ with $x_{i}$ in $\hat{J}$, we can drop the condition $\left|x_{i}-x_{j}\right| \leq \delta$. Indeed $A_{0212111}=A_{02121111}+A_{0212112}$, with

$$
\begin{aligned}
& A_{02121111}=K_{\varepsilon}^{-3} \sum_{i, j} d\left(m_{i}\right)\left|\nabla u^{\varepsilon}\left(x_{i}-x_{j} ; m_{i}, m_{j}\right)\right|^{2} \\
& \tilde{J}\left(x_{i}, m_{i}, x_{i}, m_{j}\right)^{2} \mathbb{1}\left(\left|x_{i}-x_{j}\right| \leq 1\right), \\
& \mathbb{E}_{\varepsilon}^{e q}\left|A_{02121112}\right| \leq c_{3} K^{-1} \delta^{-2} .
\end{aligned}
$$

We now choose $\delta=|\log \varepsilon|^{-1 / 3}$. In summary, $A_{02121}=A_{0212111}+E r r_{2}$, where

$$
\mathbb{E}_{\varepsilon}^{e q}\left|E r r_{2}\right| \leq c_{4}|\log \varepsilon|^{-1 / 3}
$$

On the other hand, by Lemma 5.1,

$$
\begin{aligned}
& \lim _{\varepsilon \rightarrow 0} \mathbb{E}_{\varepsilon}^{e q}\left|\int_{0}^{t} 2 A_{00}(\omega(s)) d s-t A_{0}(J)\right|=0, \\
& \lim _{\varepsilon \rightarrow 0} \mathbb{E}_{\varepsilon}^{e q}\left|\int_{0}^{t} 2 A_{02121111}(\omega(s)) d s-t A_{0}^{\prime}(J)\right|=0,
\end{aligned}
$$

where

$$
\begin{aligned}
& A_{0}(J)=2 \sum_{n} d(n) \lambda_{n} \int\left|\nabla J_{n}\right|^{2} d x \\
& A_{0}^{\prime}(J)=\sum_{m, n} \lambda_{m} \lambda_{n} d(m) \gamma(m, n) \int\left|J_{m+n}-J_{n}-J_{m}\right|^{2} d x,
\end{aligned}
$$


with

$$
\gamma(m, n)=\lim _{\varepsilon \rightarrow 0}|\log \varepsilon|^{-1} \int_{|a| \leq 1}\left|\nabla u^{\varepsilon}(a ; m, n)\right|^{2} d a .
$$

Step 2: We now study $A_{c}$. Recall that $\bar{u}^{\varepsilon}=\tilde{u}^{\varepsilon}-u^{\varepsilon}$. We have

$A_{c}(\omega)=\frac{1}{2} K_{\varepsilon}^{-1} \sum_{i, j} \alpha\left(m_{i}, m_{j}\right) V_{\varepsilon}\left(x_{i}-x_{j} ; m_{i}, m_{j}\right)\left\{S(i, j, \omega)+\sum_{r=0}^{8} R_{r}(i, j, \omega)\right\}^{2}$,

where

$$
\begin{aligned}
S(i, j, \omega) & =\tilde{J}\left(x_{i}, m_{i}, x_{j}, m_{j}\right)+\hat{J}\left(x_{i}, m_{i}, x_{j}, m_{j}\right) K_{\varepsilon}^{-1} u^{\varepsilon}\left(x_{i}-x_{j} ; m_{i}, m_{j}\right), \\
R_{0}(i, j, \omega) & =-K_{\varepsilon}^{-1} \hat{J}\left(x_{i}, m_{i}, x_{j}, m_{j}\right) \tilde{u}^{\varepsilon}\left(x_{i}-x_{j} ; m_{i}, m_{j}\right), \\
R_{1}(i, j, \omega) & =K_{\varepsilon}^{-1} \sum_{k} \frac{m_{i}}{m_{i}+m_{j}} \bar{u}^{\varepsilon}\left(x_{i}-x_{k} ; m_{i}+m_{j}, m_{k}\right) \hat{J}\left(x_{i}, m_{i}+m_{j}, x_{k}, m_{k}\right), \\
R_{2}(i, j, \omega) & =K_{\varepsilon}^{-1} \sum_{k} \frac{m_{i}}{m_{i}+m_{j}} \bar{u}^{\varepsilon}\left(x_{k}-x_{i} ; m_{k}, m_{i}+m_{j}\right) \hat{J}\left(x_{k}, m_{k}, x_{i}, m_{i}+m_{j}\right), \\
R_{3}(i, j, \omega) & =K_{\varepsilon}^{-1} \sum_{k} \frac{m_{j}}{m_{i}+m_{j}} \bar{u}^{\varepsilon}\left(x_{j}-x_{k} ; m_{i}+m_{j}, m_{k}\right) \hat{J}\left(x_{j}, m_{i}+m_{j}, x_{k}, m_{k}\right), \\
R_{4}(i, j, \omega) & =K_{\varepsilon}^{-1} \sum_{k} \frac{m_{j}}{m_{i}+m_{j}} \bar{u}^{\varepsilon}\left(x_{k}-x_{j} ; m_{k}, m_{i}+m_{j}\right) \hat{J}\left(x_{k}, m_{k}, x_{j}, m_{i}+m_{j}\right), \\
R_{5}(i, j, \omega) & =-K_{\varepsilon}^{-1} \sum_{k} \bar{u}^{\varepsilon}\left(x_{i}-x_{k} ; m_{i}, m_{k}\right) \hat{J}\left(x_{i}, m_{i}, x_{k}, m_{k}\right), \\
R_{6}(i, j, \omega) & =-K_{\varepsilon}^{-1} \sum_{k} \bar{u}^{\varepsilon}\left(x_{k}-x_{i} ; m_{k}, m_{i}\right) \hat{J}\left(x_{k}, m_{k}, x_{i}, m_{i}\right), \\
R_{7}(i, j, \omega) & =-K_{\varepsilon}^{-1} \sum_{k} \bar{u}^{\varepsilon}\left(x_{j}-x_{k} ; m_{j}, m_{k}\right) \hat{J}\left(x_{j}, m_{j}, x_{k}, m_{k}\right), \\
R_{8}(i, j, \omega) & =-K_{\varepsilon}^{-1} \sum_{k} \bar{u}^{\varepsilon}\left(x_{k}-x_{j} ; m_{k}, m_{j}\right) \hat{J}\left(x_{k}, m_{k}, x_{j}, m_{j}\right),
\end{aligned}
$$

where the summation is over $k$ with $k \neq i, j$. Let us write $T(i, j, \omega)=\sum_{r=0}^{8} R_{r}(i, j, \omega)$. We then write

$$
A_{c}(\omega)=A_{c 0}(\omega)+A_{c 1}(\omega)+A_{c 2}(\omega)
$$

with

$$
\begin{aligned}
& A_{c 0}(\omega)=\frac{1}{2} K_{\varepsilon}^{-1} \sum_{i, j} \alpha\left(m_{i}, m_{j}\right) V_{\varepsilon}\left(x_{i}-x_{j} ; m_{i}, m_{j}\right) S(i, j, \omega)^{2}, \\
& A_{c 1}(\omega)=K_{\varepsilon}^{-1} \sum_{i, j} \alpha\left(m_{i}, m_{j}\right) V_{\varepsilon}\left(x_{i}-x_{j} ; m_{i}, m_{j}\right) S(i, j, \omega) T(i, j, \omega), \\
& A_{c 2}(\omega)=\frac{1}{2} K_{\varepsilon}^{-1} \sum_{i, j} \alpha\left(m_{i}, m_{j}\right) V_{\varepsilon}\left(x_{i}-x_{j} ; m_{i}, m_{j}\right) T(i, j, \omega)^{2} .
\end{aligned}
$$


Our goal is showing that both $A_{c 1}$ and $A_{c 2}$ are small. We start with the latter. We use the inequality $\left(\sum_{0}^{8} R_{r}\right)^{2} \leq 9 \sum_{0}^{8} R_{r}^{2}$, to assert that $A_{c 2} \leq 9 \sum_{0}^{8} A_{c 2 r}$. We only bound $A_{c 20}$ and $A_{c 25}$ as the remaining $A_{c 2 r}$ are similar to $A_{c 25}$.

To bound $A_{c 25}$, we simply square out the summation in $k$ to obtain

$$
\begin{aligned}
A_{c 25}= & \frac{1}{2} K_{\varepsilon}^{-3} \sum_{i, j, k, l} \alpha\left(m_{i}, m_{j}\right) V_{\varepsilon}\left(x_{i}-x_{j} ; m_{i}, m_{j}\right) \\
& \bar{u}^{\varepsilon}\left(x_{i}-x_{k} ; m_{i}, m_{k}\right) \bar{u}^{\varepsilon}\left(x_{i}-x_{l} ; m_{i}, m_{l}\right) \\
& \hat{J}\left(x_{i}, m_{i}, x_{k}, m_{k}\right) \hat{J}\left(x_{i}, m_{i}, x_{l}, m_{l}\right)=: A_{c 251}+A_{c 252},
\end{aligned}
$$

where $A_{c 251}$ and $A_{c 252}$ represent the cases $k \neq l$ and $k=l$ respectively. We then have

$$
\begin{aligned}
\mathbb{E}_{\varepsilon}^{e q} A_{c 251} & \leq c_{1} \sum_{m, n, p, q} \alpha(m, n) \lambda_{m} \lambda_{n} \lambda_{p} \lambda_{q} \int_{|a| \leq 1}\left|\bar{u}^{\varepsilon}(a ; m, p)\right| d a \int_{|a| \leq 1}\left|\bar{u}^{\varepsilon}(a ; m, q)\right| d a \\
& \leq c_{2} \delta(\varepsilon)^{2}
\end{aligned}
$$

by (6.5). In the same fashion, we may use (6.7) to show that $\mathbb{E}_{\varepsilon}^{e q} A_{c 252} \leq c_{2} K_{\varepsilon}^{-1}$. Treating other $A_{c 2 r}, r=1, \ldots, 8$ in the same way yields

$$
\mathbb{E}_{\varepsilon}^{e q} \sum_{r=1}^{8} A_{c 2 r} \leq c_{3}\left(\delta(\varepsilon)^{2}+K_{\varepsilon}^{-1}\right) .
$$

As for $A_{c 20}$ we have that $\mathbb{E}_{\varepsilon}^{e q} A_{c 20}$ is bounded by

$$
\begin{aligned}
& \frac{1}{2} \mathbb{E}_{\varepsilon}^{e q} K_{\varepsilon}^{-3} \sum_{i, j} \alpha\left(m_{i}, m_{j}\right) V_{\varepsilon}\left(x_{i}-x_{j} ; m_{i}, m_{j}\right) \tilde{u}^{\varepsilon}\left(x_{i}-x_{j} ; m_{i}, m_{j}\right)^{2} \hat{J}\left(x_{i}, m_{i}, x_{j}, m_{j}\right)^{2} \\
& \quad \leq c_{4} K_{\varepsilon}^{-1} \sum_{m, n} \alpha(m, n) \lambda_{m} \lambda_{n} \int V_{\varepsilon}(a ; m, n) \tilde{u}^{\varepsilon}(a ; m, n)^{2} d a .
\end{aligned}
$$

If we restrict the summation to those $m$ and $n$ such that $r(m, n) \varepsilon \geq \delta(\varepsilon)$, then we simply use $\tilde{u}^{\varepsilon} \leq c_{1} K^{\varepsilon}$ to show that the sum is bounded by a constant multiple of $\tau(\varepsilon)$, which is small by our assumption (3.9). On the other hand, when $V_{\varepsilon}(a ; m, n) \neq 0$ and $r(m, n) \varepsilon \leq \delta(\varepsilon)$,

$$
\begin{aligned}
\left|\tilde{u}^{\varepsilon}(a ; m, n)\right| & =\left|\iint u^{\varepsilon}(a+z ; m, n) \zeta^{\delta(\varepsilon)}(z) d z\right| \\
& \leq c_{5} \delta(\varepsilon)^{-2} \int_{|z| \leq c_{5} \delta(\varepsilon)}\left|u^{\varepsilon}(a+z ; m, n)\right| d z \\
& \leq c_{6} \delta(\varepsilon)^{-2} \alpha^{\prime}(m, n) \int_{|z| \leq c_{5} \delta(\varepsilon)+c_{6} \varepsilon r(m, n)}|\log | z|| d z \\
& \leq c_{7} \alpha^{\prime}(m, n)|\log \delta(\varepsilon)| .
\end{aligned}
$$

Hence

$$
\mathbb{E}_{\varepsilon}^{e q} A_{c 20} \leq c_{8}|\log \varepsilon|^{-2}|\log \delta(\varepsilon)|^{2}+c_{8} \tau(\varepsilon)
$$


From this and (8.27) we deduce

$$
\mathbb{E}_{\varepsilon}^{e q} A_{c 2} \leq c_{9}\left(\delta(\varepsilon)^{2}+|\log \varepsilon|^{-1}+|\log \varepsilon|^{-2}|\log \delta(\varepsilon)|^{2}+\tau(\varepsilon)\right) .
$$

Step 3: We now turn to $A_{c 1}$. By Lemma 6.2, the expression $K_{\varepsilon}^{-1} u^{\varepsilon}(a ; m, n)$ is uniformly bounded whenever $V_{\varepsilon}(a ; m, n) \neq 0$. Hence

$$
\left|A_{c 1}\right| \leq A_{c 1}^{\prime}=c_{1} K_{\varepsilon}^{-2} \sum_{i, j, k} \alpha\left(m_{i}, m_{j}\right) V_{\varepsilon}\left(x_{i}-x_{j} ; m_{i}, m_{j}\right)|T(i, j, \omega)| .
$$

Again using the decomposition of $T$, we write $A_{c 1}^{\prime}=\sum_{r=0}^{8} A_{c 1 r}$, with for example

$$
\begin{array}{r}
A_{c 15}(\omega)=c_{1} K_{\varepsilon}^{-2} \sum_{i, j, k} \alpha\left(m_{i}, m_{j}\right) V_{\varepsilon}\left(x_{i}-x_{j} ; m_{i}, m_{j}\right) \\
\left|\bar{u}^{\varepsilon}\left(x_{i}-x_{k} ; m_{i}, m_{k}\right)\right|\left|\hat{J}\left(x_{i}, m_{i}, x_{k}, m_{k}\right)\right| .
\end{array}
$$

By (6.5),

$$
\mathbb{E}_{\varepsilon}^{e q} A_{c 15} \leq c_{2} \sum_{m, n, p} \alpha(m, n) \lambda_{m} \lambda_{n} \lambda_{p} \int_{|a| \leq 1}\left|\bar{u}^{\varepsilon}(a ; m, p)\right| d a \leq c_{2} \delta(\varepsilon) .
$$

Similarly

$$
\begin{array}{r}
A_{c 10}(\omega)=c_{1} K_{\varepsilon}^{-2} \sum_{i, j} \alpha\left(m_{i}, m_{j}\right) V_{\varepsilon}\left(x_{i}-x_{j} ; m_{i}, m_{j}\right) \\
\quad\left|\tilde{u}^{\varepsilon}\left(x_{i}-x_{j} ; m_{i}, m_{j}\right)\right|\left|\hat{J}\left(x_{i}, x_{j}, m_{i}, m_{j}\right)\right|,
\end{array}
$$

which yields

$$
\mathbb{E}_{\varepsilon}^{e q} A_{c 10}(\omega) \leq c_{3} \sum_{m, n} \alpha(m, n) \lambda_{m} \lambda_{n} \int_{|a| \leq 1} V_{\varepsilon}(a ; m, n)\left|\tilde{u}^{\varepsilon}(a ; m, n)\right| d a .
$$

Again using (8.28) we obtain

$$
\mathbb{E}_{\varepsilon}^{e q} A_{c 10}(\omega) \leq c_{4}|\log \varepsilon|^{-1}|\log \delta(\varepsilon)|+c_{4} \tau(\varepsilon),
$$

in the same way we obtained (8.28). From this and (8.30) we deduce

$$
\mathbb{E}_{\varepsilon}^{e q} A_{c 1} \leq c_{5}\left(\delta(\varepsilon)+|\log \varepsilon|^{-1}|\log \delta(\varepsilon)|+\tau(\varepsilon)\right) .
$$

From this, (8.26) and (8.30) we deduce

$$
\int_{0}^{t} A_{c}(\omega(s)) d s=\int_{0}^{t} A_{c 0}(\omega(s)) d s+\operatorname{Err}_{2}
$$

with

$$
\mathbb{E}_{\varepsilon}^{e q}\left|\operatorname{Err}_{2}\right| \leq c\left(\delta(\varepsilon)+|\log \varepsilon|^{-1}+|\log \varepsilon|^{-1}|\log \delta+\tau(\varepsilon)|\right) .
$$


On the other hand, by the Law of Large Numbers,

$$
\lim _{\varepsilon \rightarrow 0} \mathbb{E}_{\varepsilon}^{e q}\left|\int_{0}^{t} A_{c 0}(\omega(s)) d s-t A_{c}(J)\right|=0,
$$

where

$$
A_{c}(J)=\frac{1}{2} \int \sum_{m, n} \tilde{\alpha}(m, n) \lambda_{n} \lambda_{m}\left(J_{n+m}-J_{n}-J_{m}\right)^{2} d x
$$

and

$$
\tilde{\alpha}(m, n)=\alpha(m, n) \eta(m, n)^{2}=\hat{\alpha}(m, n) \eta(m, n),
$$

where $\eta=\hat{\alpha} / \alpha$. Here we have used $\lim _{\varepsilon} K_{\varepsilon}^{-1} u^{\varepsilon}=\eta-1$ uniformly in the support of $V_{\varepsilon}$. (See Theorem 3.2 of $[\mathrm{HR}]$.)

Step 4: We now concentrate on $A_{f}$. We have

$$
\begin{aligned}
A_{f}(\omega)= & \frac{1}{2} K_{\varepsilon}^{-1} \sum_{i} \sum_{m=1}^{m_{i}-1} \beta\left(m, m_{i}-m\right) \int V^{\varepsilon}\left(x_{i}-y ; m_{i}-m, m\right) \\
& {\left[S(i ; y, m ; \omega)+\sum_{r=0}^{4} R_{r}(i ; y, m ; \omega)\right]^{2} d y, }
\end{aligned}
$$

where

$$
\begin{aligned}
S(i ; y, m ; \omega)= & J\left(x_{i}, m\right)+J\left(y, m_{i}-m\right)-J\left(x_{i}, m_{i}\right) \\
& -K_{\varepsilon}^{-1} u^{\varepsilon}\left(x_{i}-y ; m, m_{i}-m\right) \hat{J}\left(x_{i}, m, y, m_{i}-m\right), \\
R_{0}(i ; y, m ; \omega)= & K_{\varepsilon}^{-1} \tilde{u}^{\varepsilon}\left(x_{i}-y ; m, m_{i}-m\right) \hat{J}\left(x_{i}, m, y, m_{i}-m\right), \\
R_{1}(i ; y, m ; \omega)= & K_{\varepsilon}^{-1} \sum_{j}\left[\bar{u}^{\varepsilon}\left(x_{i}-x_{j} ; m, m_{j}\right) \hat{J}\left(x_{i}, m, x_{j}, m_{j}\right)\right. \\
& \left.-\bar{u}^{\varepsilon}\left(x_{i}-x_{j} ; m_{i}, m_{j}\right) \hat{J}\left(x_{i}, m_{i}, x_{j}, m_{j}\right)\right], \\
R_{2}(i ; y, m ; \omega)= & K_{\varepsilon}^{-1} \sum_{j}\left[\bar{u}^{\varepsilon}\left(x_{i}-x_{j} ; m_{i}, m\right) \hat{J}\left(x_{i}, m_{i}, x_{j}, m\right)\right. \\
& \left.-\bar{u}^{\varepsilon}\left(x_{i}-x_{j} ; m_{i}, m_{j}\right) \hat{J}\left(x_{i}, m_{i}, x_{j}, m_{j}\right)\right], \\
R_{3}(i ; y, m ; \omega)= & K_{\varepsilon}^{-1} \sum_{j} \bar{u}^{\varepsilon}\left(y-x_{j} ; m, m_{j}\right) \hat{J}\left(y, m_{j}, x_{j}, m_{j}\right), \\
R_{4}(i ; y, m ; \omega)= & K_{\varepsilon}^{-1} \sum_{j} \bar{u}^{\varepsilon}\left(x_{j}-y ; m_{j}, m\right) \hat{J}\left(x_{j}, m_{j}, y, m\right) .
\end{aligned}
$$

Let us write $T=\sum_{0}^{4} R_{r}$, and

$$
A_{f}=A_{f 0}+A_{f 1}+A_{f 2},
$$


with

$$
\begin{aligned}
& A_{f 0}=\frac{1}{2} K_{\varepsilon}^{-1} \sum_{i} \sum_{m=1}^{m_{i}-1} \beta\left(m, m_{i}-m\right) \int V^{\varepsilon}\left(x_{i}-y ; m_{i}-m, m\right) S(i ; y, m ; \omega)^{2} d y, \\
& A_{f 1}=K_{\varepsilon}^{-1} \sum_{i} \sum_{m=1}^{m_{i}-1} \beta\left(m, m_{i}-m\right) \int V^{\varepsilon}\left(x_{i}-y ; m_{i}-m, m\right)(S T)(i ; y, m ; \omega) d y, \\
& A_{f 2}=\frac{1}{2} K_{\varepsilon}^{-1} \sum_{i} \sum_{m=1}^{m_{i}-1} \beta\left(m, m_{i}-m\right) \int V^{\varepsilon}\left(x_{i}-y ; m_{i}-m, m\right) T(i ; y, m ; \omega)^{2} d y .
\end{aligned}
$$

We have that $A_{f 2} \leq \frac{5}{2} \sum_{0}^{4} A_{f 2 r}$ with

$$
A_{f 2 r}(\omega)=K_{\varepsilon}^{-1} \sum_{i} \sum_{m=1}^{m_{i}-1} \beta\left(m, m_{i}-m\right) \int V^{\varepsilon}\left(x_{i}-y ; m_{i}-m, m\right) R_{r}(i ; y, m ; \omega)^{2} d y .
$$

We then use (8.27) to learn that $A_{f 20}(\omega)$ is bounded above by

$$
\begin{aligned}
& c_{1} K_{\varepsilon}^{-3} \sum_{i} \sum_{m=1}^{m_{i}-1} \beta\left(m, m_{i}-m\right) \int V^{\varepsilon}\left(x_{i}-y ; m_{i}-m, m\right) \tilde{u}^{\varepsilon}\left(x_{i}-y ; m, m_{i}-m\right)^{2} d y \\
& \leq c_{2} K_{\varepsilon}^{-3}|\log \delta(\varepsilon)|^{2} \sum_{i} \sum_{m=1}^{m_{i}-1} \beta\left(m, m_{i}-m\right) \alpha^{\prime}\left(m, m_{i}-m\right)^{2} .
\end{aligned}
$$

We then use (3.10) to deduce

$$
\mathbb{E}_{\varepsilon}^{e q} A_{f 20}(\omega) \leq c_{3}|\log \varepsilon|^{-2}|\log \delta(\varepsilon)|^{2} .
$$

On the other hand,

$$
A_{f 21}(\omega)=K_{\varepsilon}^{-1} \sum_{i} \sum_{m=1}^{m_{i}-1} \beta\left(m, m_{i}-m\right) R_{1}(i ; y, m ; \omega)^{2}
$$

is bounded above by $A_{f 211}+A_{f 212}$, with

$$
\begin{aligned}
& A_{f 211}=2 K_{\varepsilon}^{-3} \sum_{i} \sum_{m=1}^{m_{i}-1} \beta\left(m, m_{i}-m\right)\left[\sum_{j}\left|\bar{u}^{\varepsilon}\left(x_{i}-x_{j} ; m, m_{j}\right) \hat{J}\left(x_{i}, m, x_{j}, m_{j}\right)\right|\right]^{2} \\
& A_{f 212}=2 K_{\varepsilon}^{-3} \sum_{i} \sum_{m=1}^{m_{i}-1} \beta\left(m, m_{i}-m\right)\left[\sum_{j}\left|\bar{u}^{\varepsilon}\left(x_{i}-x_{j} ; m_{i}, m_{j}\right) \hat{J}\left(x_{i}, m_{i}, x_{j}, m_{j}\right)\right|\right]^{2} .
\end{aligned}
$$

By squaring out the expression inside brackets, we can readily see

$$
A_{f 211}+A_{f 212} \leq c_{1}\left(\delta(\varepsilon)^{2}+K_{\varepsilon}^{-1}\right)
$$


by Lemma 6.1. The term $A_{f 22}$ is treated likewise. Hence,

$$
\mathbb{E}_{\varepsilon}^{e q}\left(A_{f 21}(\omega)+A_{f 22}(\omega)\right) \leq c_{2}\left(\delta(\varepsilon)^{2}+K_{\varepsilon}^{-1}\right)
$$

We now study $A_{f 23}$ :

$$
\begin{aligned}
A_{f 23}(\omega)= & K_{\varepsilon}^{-3} \sum_{i, j, k} \sum_{m=1}^{m_{i}-1} \beta\left(m, m_{i}-m\right) \int V^{\varepsilon}\left(x_{i}-y ; m_{i}-m, m\right) \\
& \bar{u}^{\varepsilon}\left(y-x_{j} ; m, m_{j}\right) \hat{J}\left(y, m, x_{j}, m_{j}\right) \bar{u}^{\varepsilon}\left(y-x_{k} ; m, m_{k}\right) \hat{J}\left(y, m, x_{k}, m_{k}\right) d y \\
= & A_{f 231}+A_{f 232},
\end{aligned}
$$

with $A_{f 231}$ and $A_{f 232}$ corresponding to the cases $k \neq j$ and $k=j$. With the aid of (6.5) and (6.8), we can readily deduce

$$
\mathbb{E}_{\varepsilon}^{e q} A_{f 231} \leq c_{3} \delta(\varepsilon)^{2}, \quad \mathbb{E}_{\varepsilon}^{e q} A_{f 232} \leq c_{3}|\log \varepsilon|^{-1}
$$

The term $A_{f 24}$ is treated likewise. In summary

$$
E_{e q}^{\varepsilon} A_{f 2}(\omega) \leq c_{4}\left(\delta(\varepsilon)^{2}+|\log \varepsilon|^{-1}\right) .
$$

We can readily bound $A_{f 1}$ as in the previous step:

$$
E_{e q}^{\varepsilon} A_{f 1}(\omega) \leq c_{5}\left(\delta(\varepsilon)+|\log \varepsilon|^{-1}|\log d(\varepsilon)+\tau(\varepsilon)|\right) .
$$

From all this we conclude

$$
\int_{0}^{t} A_{f}(\omega(s)) d s=\int_{0}^{t} A_{f 0}(\omega(s)) d s+\operatorname{Err}_{3}
$$

with $\operatorname{Err}_{3}$ satisfying (8.32). On the other hand

$$
\begin{aligned}
S(i ; y, m ; \omega)= & -J\left(x_{i}, m, x_{i}, m_{i}-m\right) \\
& \left(1+K_{\varepsilon}^{-1} u^{\varepsilon}\left(x_{i}-y ; m, m_{i}-m\right)\right)+O\left(\varepsilon r\left(m, m_{i}-m\right)\right)
\end{aligned}
$$

whenever $V^{\varepsilon}\left(y-x_{i} ; m, m_{i}-m\right) \neq 0$. From this and a law of large numbers, we can readily deduce

$$
\lim _{\varepsilon \rightarrow 0} \mathbb{E}_{\varepsilon}^{e q}\left|\int_{0}^{t} A_{f 0}(\omega(s)) d s-t A_{f}(J)\right|=0,
$$

where

$$
A_{f}(J)=\frac{1}{2} \int \sum_{m, n} \tilde{\beta}(m, n) \lambda_{n+m}\left(J_{n+m}-J_{n}-J_{m}\right)^{2} d x
$$

where

$$
\tilde{\beta}(m, n)=\beta(m, n) \eta(m, n)^{2}=\hat{\beta}(m, n) \eta(m, n) .
$$


Final Step: From (8.22), (8.23), (8.24), (8.32), (8.35) and (8.37) we learn that the process

$$
\bar{M}_{\varepsilon}(t)^{2}-t A^{\prime}(J),
$$

is a sum of a martingale and a small error, where

$$
\begin{aligned}
A^{\prime}(J)= & 2 \int \sum_{n} \lambda_{n} d(n)\left|\nabla_{x} J_{n}\right|^{2} d x+\int \sum_{n, m} \lambda_{n} \lambda_{m} d(n) \gamma(n, m)\left(J_{n+m}-J_{n}-J_{m}\right)^{2} d x \\
& +\frac{1}{2} \int \sum_{m, n} \tilde{\alpha}(m, n) \lambda_{n} \lambda_{m}\left(J_{n+m}-J_{n}-J_{m}\right)^{2} d x \\
& +\frac{1}{2} \int \sum_{m, n} \tilde{\beta}(m, n) \lambda_{n+m}\left(J_{n+m}-J_{n}-J_{m}\right)^{2} d x .
\end{aligned}
$$

It remains to show that $A(J)=A^{\prime}(J)$. Since

$$
\lambda_{m} \lambda_{n} \tilde{\alpha}(m, n)=\tilde{\beta}(m, n) \lambda_{n+m}, \quad \lambda_{m} \lambda_{n} \hat{\alpha}(m, n)=\hat{\beta}(m, n) \lambda_{n+m},
$$

it suffices to show

$$
\begin{aligned}
(d(m)+d(n)) \gamma(m, n) & =\lim _{\varepsilon \rightarrow 0}(d(m)+d(n)) K_{\varepsilon}^{-1} \int_{|a| \leq 1}\left|\nabla u^{\varepsilon}(a ; m, n)\right|^{2} d a \\
& =\hat{\alpha}(m, n)-\tilde{\alpha}(m, n)=\alpha(m, n)\left(\eta(m, n)-\eta(m, n)^{2}\right) .
\end{aligned}
$$

We have

$$
\begin{aligned}
& (d(m)+d(n)) K_{\varepsilon}^{-1} \int_{|a| \leq 1}\left|\nabla u^{\varepsilon}(a ; m, n)\right|^{2} d a \\
& =-(d(m)+d(n)) K_{\varepsilon}^{-1} \int_{|a| \leq 1} u^{\varepsilon}(a ; m, n) \Delta u^{\varepsilon}(a ; m, n) d a+O\left(K_{\varepsilon}^{-1}\right) \\
& =-\alpha(m, n) \int_{|a| \leq 1} V^{\varepsilon}(a ; m, n) K_{\varepsilon}^{-1} u^{\varepsilon}(a ; m, n) \\
& \quad \times\left(1+K_{\varepsilon}^{-1} u^{\varepsilon}(a ; m, n)\right) d a+O\left(K_{\varepsilon}^{-1}\right),
\end{aligned}
$$

where we integrated by parts and used (4.11). This and $\lim _{\varepsilon} K_{\varepsilon}^{-1} u^{\varepsilon}=\eta-1$ (Theorem 3.2 of [HR1]) imply (8.38).

\section{Proofs of Theorems 8.1 and 3.1}

In this section, we complete the proof of Theorems 8.1 and 3.1. We first show that the process $\xi^{\prime}(t, J)$ is tight. More precisely,

Theorem 9.1. For every smooth function $J$ of compact support and positive $T$, there exists a constant $c(J, T)$ such that

$$
\limsup _{\varepsilon \rightarrow 0} \mathbb{E}_{\varepsilon}^{e q} \int_{0}^{T} \sup _{0 \leq h \leq \delta}\left|\xi^{\prime}(t+h, J)-\xi^{\prime}(t, J)\right| d t \leq c(J, T) \delta^{1 / 2} .
$$


Proof. Recall that by (8.16),

$$
\xi^{\prime}(t, J)-\xi^{\prime}(s, J)=\int_{s}^{t} \Gamma \xi(\theta, J) d \theta+\bar{M}_{\varepsilon}(t)-\bar{M}_{\varepsilon}(s)-\int_{s}^{t} \operatorname{Err}_{8}(\omega(\theta)) d \theta,
$$

where,

$$
\limsup _{\varepsilon \rightarrow 0} \mathbb{E}_{\varepsilon}^{e q} \sup _{0 \leq t \leq T}\left|\int_{0}^{t} \operatorname{Err}_{8}(\omega(\theta)) d \theta\right| \leq T \limsup _{\varepsilon \rightarrow 0} \mathbb{E}_{\varepsilon}^{e q}\left|\operatorname{Err}_{8}(\omega(0))\right|=0 .
$$

On the other hand, since

$$
\sup _{\varepsilon} \mathbb{E}_{\varepsilon}^{e q} \Gamma \xi(\theta, J)^{2}=\sup _{\varepsilon} \mathbb{E}_{\varepsilon}^{e q} \Gamma \xi(0, J)^{2}<\infty,
$$

we can readily deduce

$$
\begin{aligned}
& \mathbb{E}_{\mathcal{\varepsilon}}^{e q} \sup _{0 \leq s \leq t \leq s+\delta \leq T}\left|\int_{s}^{t} \Gamma \xi(\theta, J) d \theta\right| \\
& \leq(T+\delta)^{1 / 2}\left[\mathbb{E}_{\varepsilon}^{e q} \int_{s}^{t} \Gamma \xi(\theta, J)^{2} d \theta\right]^{1 / 2} \leq c_{1} \delta^{1 / 2} .
\end{aligned}
$$

See Sect. 5 and (5.7) of [R] for more details.

It remains to establish the tightness of the martingale $\bar{M}_{\varepsilon}$. For this, it suffices to show

$$
\lim _{\varepsilon \rightarrow 0} \sup _{0 \leq t \leq T} \sup _{0<h<\delta} \mathbb{E}_{\varepsilon}^{e q}\left[\bar{M}_{\varepsilon}(t+h)-\bar{M}_{\varepsilon}(t)\right]^{2} \leq c_{0}(T) \delta .
$$

This is an immediate consequence of Doob's inequality and (8.5). From (9.2), (9.3) and (9.5) we deduce (9.1).

We are now ready to complete the proof of Theorems 3.1 and 8.1. Take a countable set $\left\{t_{n}: n \in \mathbb{N}\right\}$ that contains 0 and is dense in some fixed interval $[0, T]$. Write $\tilde{\mathcal{P}}^{\varepsilon}$ for the law of

$$
\left(\left(\xi^{\prime}(\cdot, J): J \in \mathcal{D}_{0}\right),\left(\xi^{\prime}\left(t_{n}, J\right): J \in \mathcal{D}_{0}, n \in \mathbb{N}\right)\right) \in \mathcal{H} \times \mathbb{R}^{\mathcal{D}_{0} \times \mathbb{N}},
$$

with respect to the probability measure $\mathbb{P}_{\varepsilon}^{e q}$. Using Theorem 9.1 , we can readily show that the sequence $\tilde{\mathcal{P}}^{\varepsilon}$ is tight. Let $\tilde{\mathcal{P}}$ be a limit point of the sequence $\tilde{\mathcal{P}}^{\varepsilon}$. Using Theorem 8.2, it is not hard to show that for every $J \in \mathcal{D}_{0}$, the sequences

$$
\begin{aligned}
& \left(M_{J}\left(t_{n}\right):=\xi^{\prime}\left(t_{n}, J\right)-\xi^{\prime}(0, J)-\int_{0}^{t_{n}} \Gamma \xi^{\prime}(s, J) d s: n \in \mathbb{N}\right), \\
& \left(N_{J}\left(t_{n}\right):=M_{J}\left(t_{n}\right)^{2}-t_{n} A(J): n \in \mathbb{N}\right),
\end{aligned}
$$

are martingales with respect to the probability measure $\tilde{\mathcal{P}}$. We now extend $M_{J}$ and $N_{J}$ to the whole interval $[0, T]$. More precisely, for $t \notin\left\{t_{n}: n \in \mathbb{N}\right\}$, define

$$
M_{J}(t):=\lim _{t_{n} \rightarrow t-} M_{J}\left(t_{n}\right), \quad N_{J}(t):=\lim _{t_{n} \rightarrow t-} N_{J}\left(t_{n}\right),
$$

which exist almost surely by the Martingale Upcrossing Theorem. Here by $\lim _{t_{n} \rightarrow t-}$, we mean the limit with respect to a subsequence of $\left\{t_{n}\right\}$ which increases to $t$ from the 
left. As a result, $\bar{\xi}(t, J):=\lim _{t_{n} \rightarrow t-} \xi\left(t_{n}, J\right)$ also exists almost surely with respect to $\tilde{\mathcal{P}}$. The process $\bar{\xi}(t, J)$ is a solution to (3.13) in the sense of Definition 8.1. This completes the proof of Theorem 3.1 because the set $\left\{t_{n}: n \in \mathbb{N}\right\}$ can be chosen to include any given finite collection of points.

To complete the proof of Theorem 8.1, it suffices to show that $\xi^{\prime}(t, J)=\bar{\xi}(t, J)$ almost everywhere. For this, let us assume that the set $\left\{t_{n}: n \in \mathbb{N}\right\}$ includes the points in the set $\{i / L: i \in \mathbb{N}\} \cap[0, T]$ for every positive integer $L$. Write $T_{L}(t)$ for $[t L] / L$, where $[t L]$ denotes the integer part of $t L$. From (9.1) we can readily deduce

$$
\iint_{0}^{T}\left|\xi^{\prime}(t, J)-\xi^{\prime}\left(T_{L}(t), J\right)\right| d t d \tilde{\mathcal{P}} \leq c(J, T) L^{-1 / 2} .
$$

Since $\lim _{L \rightarrow \infty} \xi^{\prime}\left(T_{L}(t)\right)=\bar{\xi}(t, J)$ by definition, we deduce that $\xi^{\prime}(t, J)=\bar{\xi}(t, J)$ for almost all $t$ and almost surely.

Open Access This article is distributed under the terms of the Creative Commons Attribution Noncommercial License which permits any noncommercial use, distribution, and reproduction in any medium, provided the original author(s) and source are credited.

\section{References}

[A] Aldous, D.J.: Deterministic and stochastic models for coalescence (aggregation, coagulation): a review of the mean-field theory for probabilists. Bernoulli 5, 3-48 (1999)

[C] Chang, C.C.: Equilibrium fluctuations of gradient reversible particle systems. Prob. Th. Rel. Fields 100, 269-283 (1994)

[CY] Chang, C.C., Yau, H.T.: Fluctuations of one-dimensional ginzburg-landau models in nonequilibrium. Commun. Math. Phys. 145, 209-234 (1992)

[EK] Ethier, S.N., Kurtz, T.G.: Markov Processes. Characterization and Convergence. Wiley, New York, 1986

[HR1] Hammond, A.M., Rezakhanlou, F.: Kinetic limit for a system of coagulating planar Brownian particles. J. Stat. Phys. 123(2-4), 997-1040 (2006)

[HR2] Hammond, A.M., Rezakhanlou, F.: The kinetic limit of a system of coagulating Brownian particles. Arch. Rat. Mech. Anal. 185, 1-67 (2007)

[HR3] Hammond, A.M., Rezakhanlou, F.: Moment bounds for the Smoluchowski equation and their consequences. Commun. Math. Phys. 276, 645-670 (2007)

[HS] Holley, R.A., Stroock, D.W.: Generalized ornstein-uhlenbeck processes and infinite particle branching Brownian motions. Publ. Res. Inst. Math. Sci. 14, 741-788 (1978)

[KV] Kipnis, C., Varadhan, S.R.S.: Central limit theorem for additive functionals of reversible markov processes and applications to simple exclusions. Comm. Math. Phys. 104, 1-19 (1986)

[LN] Lang, R., Nyugen, X.-X.: Smoluchowski's theory of coagulation in colloids holds rigorously in the Boltzmann-grad limit. Z. Wahrsch. Verw. Gebiete 54, 227-280 (1980)

[R1] Rezakhanlou, F.: Equilibrium fluctuations for the discrete Boltzmann equation. Duke J. Math. 93(2), 257-288 (1998)

[R2] Rezakhanlou, F.: The coagulating Brownian particles and Smoluchowski's equation. Markov Process. Rel. Fields 12, 425-445 (2006)

[Sm] Smoluchowski, M.: Drei vortrage uber diffusion, brown'sche molekular bewegung und koagulation von kolloidteilchen. Phys. Z. XVII, 557-571, 585-599 (1916)

[Sz] Sznitman, A.S.: Propagation of chaos for a system of annihilating Brownian spheres. Comm. Pure Appl. Math. 40, 663-690 (1987)

[Sp] Spohn, H.: Large Scale Dynamics of Interacting Particles. Springer, Berlin-Hidelberg-NewYork (1991)

[HRY] Yaghouti, M., Rezakhanlou, F., Hammond, A.M.: Coagulation, diffusion and the continuous smoluchowski equation. Stoch. Proc. Appl. 119, 3042-3080 (2009)

Communicated by H. Spohn 